\title{
Global Phase Diagram of a Dirty Weyl Liquid and Emergent Superuniversality
}

\author{
Bitan Roy, ${ }^{1,2,3}$ Robert-Jan Slager, ${ }^{3}$ and Vladimir Juričić ${ }^{4}$ \\ ${ }^{1}$ Condensed Matter Theory Center and Joint Quantum Institute, University of Maryland, \\ College Park, Maryland 20742, USA \\ ${ }^{2}$ Department of Physics and Astronomy, Rice University, Houston, Texas 77005, USA \\ ${ }^{3}$ Max-Planck-Institut für Physik komplexer Systeme, Nöthnitzer Strasse 38, 01187 Dresden, Germany \\ ${ }^{4}$ Nordita, KTH Royal Institute of Technology and Stockholm University, \\ Roslagstullsbacken 23, 10691 Stockholm, Sweden
}

(Received 13 August 2017; published 19 September 2018)

\begin{abstract}
Pursuing complementary field-theoretic and numerical methods, we here paint the global phase diagram of a three-dimensional dirty Weyl system. The generalized Harris criterion, augmented by a perturbative renormalization-group analysis shows that weak disorder is an irrelevant perturbation at the Weyl semimetal (WSM)-insulator quantum-critical point. But, a metallic phase sets in through a quantum phase transition (QPT) at strong disorder across a multicritical point. The field-theoretic predictions for the correlation length exponent $\nu=2$ and dynamic scaling exponent $z=5 / 4$ at this multicritical point are in good agreement with the ones extracted numerically, yielding $\nu=1.98 \pm 0.10$ and $z=1.26 \pm 0.05$, from the scaling of the average density of states (DOS). Deep inside the WSM phase, generic disorder is also an irrelevant perturbation, while a metallic phase appears at strong disorder through a QPT. We here demonstrate that in the presence of generic but strong disorder, the WSM-metal QPT is ultimately always characterized by the exponents $\nu=1$ and $z=3 / 2$ (to one-loop order), originating from intranode or chiralsymmetric (e.g., regular and axial potential) disorder. We here anchor such emergent chiral superuniversality through complementary renormalization-group calculations, controlled via $\epsilon$ expansions, and numerical analysis of average DOS across WSM-metal QPT. In addition, we also discuss a subsequent QPT (at even stronger disorder) of a Weyl metal into an Anderson insulator by numerically computing the typical DOS at zero energy. The scaling behavior of various physical observables, such as residue of quasiparticle pole, dynamic conductivity, specific heat, Grüneisen ratio, inside various phases as well as across various QPTs in the global phase diagram of a dirty Weyl liquid, are discussed.
\end{abstract}

DOI: 10.1103/PhysRevX.8.031076

\section{INTRODUCTION}

The complex energy landscape of electronic quantummechanical states in solid-state compounds, commonly known as band structure, can display accidental or symmetry-protected band touching at isolated points in the Brillouin zone [1-9]. In the vicinity of such diabolic points, low-energy excitations can often be described as quasirelativistic Dirac or Weyl fermions [10-12], which may provide an ideal platform for condensed matter realization of various peculiar phenomena, such as chiral anomaly, Casimir effect, and axionic electrodynamics [13-15]. Recently, three-dimensional Weyl semimetals

Published by the American Physical Society under the terms of the Creative Commons Attribution 4.0 International license. Further distribution of this work must maintain attribution to the author(s) and the published article's title, journal citation, and DOI.

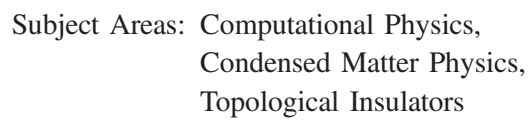

(WSMs) have attracted a lot of interest due to the growing evidence of their material realization [16-25].

A WSM, the prime example of a gapless topological phase of matter, is constituted by so-called Weyl nodes that in the reciprocal space (Brillouin zone) act as the source and sinks of Abelian Berry curvature, and thus always appear in pairs [26]. In a nutshell, the Abelian Berry flux enclosed by the system determines the integer topological invariant of a WSM and the degeneracy of topologically protected surface Fermi arcs. A question of fundamental and practical importance in this context concerns the stability of such a gapless topological phase against impurities or disorder, inevitably present in real materials. Combining complementary field-theoretic renormalizationgroup (RG) calculations and a numerical analysis of the average density of states (ADOS), we here study the role of randomness in various regimes of the phase diagram of a Weyl system to arrive at the global phase diagram, schematically illustrated in Fig. 1. 


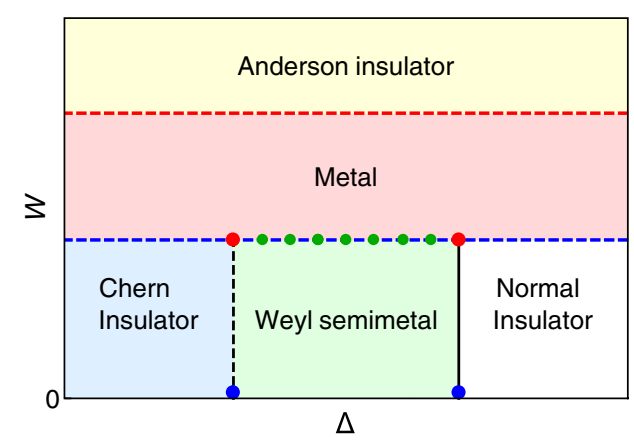

FIG. 1. A schematic phase diagram of a dirty Weyl semimetal. Here, $\Delta$ is a tuning parameter that drives quantum phase transition from Weyl semimetal to (Chern or normal) insulator in clean system [see Sec. II], and $W$ denotes the strength of disorder (the nature of which is not specified here). Semimetalinsulator quantum-critical points are denoted by the blue dots. The red dots represent multicritical points, where an insulator, a metal, and the Weyl semimetal meet [see Sec. III]. The string of green dots represents a line of quantum-critical points through one of which (depending on the bare value of $\Delta$ ) the Weyl semimetal undergoes a quantum phase transition into a metallic phase (see Secs. IV, V, VI, and VII). At stronger disorder the metallic phase undergoes a second quantum phase transition into the Anderson insulator phase (see Sec. IX). The shape of the phase boundaries is, however, nonuniversal. See, for example, Fig. 2 for numerically obtained phase diagram from a lattice model.

A WSM can be constructed by appropriately stacking two-dimensional layers of quantum anomalous Hall insulator (QAHI) in the momentum space along the $k_{z}$ direction, for example. Thus, by construction a WSM inherits the two-dimensional integer topological invariant of constituting layers of QAHI, and the momentum space Skyrmion number of QAHI jumps by an integer amount across two Weyl nodes. As a result, the Weyl nodes serve as the sources and sinks for Abelian Berry curvature, and in a clean system WSM is sandwiched between a topological Chern and a trivial insulating phase, as shown in Fig. 1. In an effective tight-binding model a WSM-insulator quantum phase transition (QPT), the blue dot in Fig. 1, can be tuned by changing the effective hopping in the $k_{z}$ direction, as demonstrated in Sec. II. In this work, we first assess the stability of such a clean semimetal-insulator quantumcritical point $(\mathrm{QCP})$ in the presence of generic randomness in the system, and arrive at the following conclusions.

(1) By generalizing the Harris criterion [27], we find that WSM-insulator QCP is stable against sufficiently weak, but otherwise generic, disorder (see Sec. III). Such an outcome is further substantiated from the scaling analysis of disorder couplings, suggesting that any disorder is an irrelevant perturbation at such a clean QCP.

(2) From an appropriate $\epsilon$ expansion (see Sec. III), we demonstrate that a multicritical point (MCP) emerges at stronger disorder, where the WSM, a band insulator (either Chern or trivial), and a metallic phase meet, the red dot in Fig. 1. The critical semimetal residing at the phase boundary between a WSM and an insulator (along the black dashed line in Fig. 1) then becomes unstable toward the formation of a compressible metal through such a MCP. The exponents capturing the instability of critical excitations toward the onset of a metal are (a) correlation length exponent (CLE) $\nu=2$ and (b) dynamic scaling exponent (DSE) $z=5 / 4$ to the leading order in the $\epsilon$ expansion. These two exponents also determine the scaling behavior of physical observables across the anisotropic critical semimetal-metal QPT.

(3) By following the scaling of DOS along the phase boundary (the black dashed line in Fig. 1) between the WSM and insulator with increasing randomness in the system, we numerically extract $\nu$ and $z$ at the MCP across the critical semimetal-metal QPT (see Fig. 2). Numerically extracted values of these two exponents are $\nu=1.98 \pm 0.10$ and $z=1.26 \pm 0.05$ (see Sec. III B), which are in good agreement with our prediction from the leading-order $\epsilon$ expansion (see Appendix E, Table IV).

We now turn our focus on the WSM phase (the green shaded region in Fig. 1). The study of disorder effects in topological phases of matter has recently attracted a lot of attention, leading to a surge of analytical [28-50] and numerical [51-65] works. In particular, the focus has been concentrated on the massless Dirac critical point separating two topologically distinct insulators (electrical or thermal), as well as inside Dirac and Weyl semimetal phases. Even though the effects of generic disorders have been studied to some extent theoretically [30,36,42-44], most of the numerical works solely focused on random charge impurities (for exceptions, see Refs. [54,56]). By now there is both analytical and numerical evidence that chemical potential disorder when strong enough drives a QPT from the WSM to a diffusive metal, leaving its imprint on different observables, e.g., average DOS, specific heat, and conductivity (see Sec. VIII). Deep inside the WSM phase, the system possesses various emergent symmetries (see Table III), such as a continuous global chiral U(1) symmetry that is tied with the translational symmetry of a clean noninteracting WSM in the continuum limit [66]. In the absence of both inversion and time-reversal symmetries, the simplest realization of a WSM with only two Weyl nodes is susceptible to 16 possible sources of elastic scattering, displayed in Table III. They can be grouped in eight classes, among which only four preserve the emergent global chiral symmetry (intranode scattering), while the remaining ones directly mix two Weyl nodes with opposite (left and right) chiralities (internode scattering) [67]. As we demonstrate in this paper, such characterization of disorders based on the 

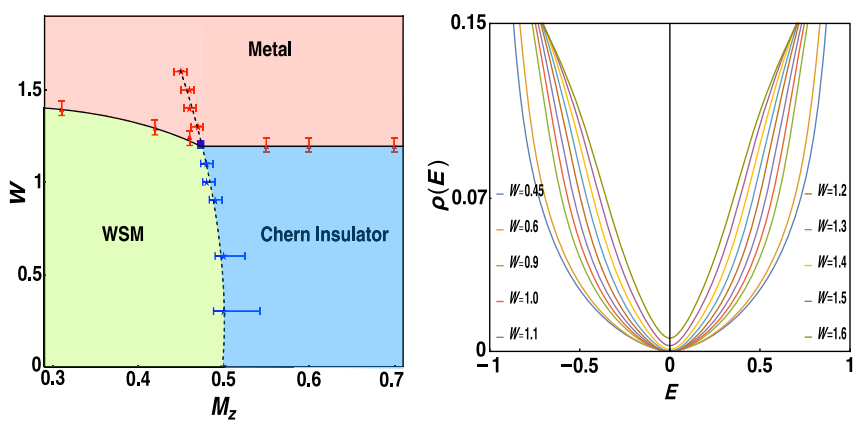

FIG. 2. Left: Numerically obtained phase diagram of a Weyl material residing in the proximity of the WSM-insulator QCP (blue dot for $W=0$ ) in the presence of random charge impurities $(W)$. The black dashed line represents the phase boundary between these two phases, and the blue square is the multicritical point (MCP) where the WSM, a band insulator (Chern in the present situation), and a metal meet. The density of states at the phase semimetal-insulator phase boundary scales as $\varrho(E) \sim|E|^{1.5}$, as shown in the right-hand panel (see Sec. III for details). With increasing strength of disorder the direct transition between WSM and insulator gets avoided by an intervening metallic phase, where DOS at zero energy is finite (see the right-hand panel). The metallicity sets in through the MCP, where the DOS scales roughly as $\varrho(E) \sim|E|$. These findings are in qualitative agreement with the field-theoretic predictions (see Fig. 6).

chiral symmetry allows us to classify the WSM-metal QPTs (across one of the green dots shown in Fig. 1) in the presence of generic disorder.

To motivate our theoretical analysis, we now discuss the possible microscopic origin of disorders in the Weyl materials. Furthermore, knowing this in the future may facilitate a control over randomness in experiments on these materials. For example, chemical potential disorder can be controlled by modifying the concentration of random charge impurities. Random asymmetric shifts of chemical potential between the left and right chiral Weyl cones correspond to the axial potential disorder. Therefore, in an inversion asymmetric WSM such disorder is always present. Magnetic disorder is yet another type of chiral symmetry-preserving (CSP) disorder, and the strength of random magnetic scatterers can be efficiently tuned by systematically injecting magnetic ions in the system. [We here do not consider Kondo effect or Ruderman-KittelKasuya-Yosida (RKKY) interaction.] In contrast, all chiral-symmetry-breaking (CSB) disorders cause mixing of two Weyl nodes, and in an effective model for WSMs, they stem from various types of random bond disorder that also cause random fluctuation of bandwidth (see Appendix D). Therefore, strength of CSB disorder may be tuned by applying inhomogeneous pressure (hydrostatic or chemical) in the Weyl materials. Since the WSMs are found in strong spin-orbit-coupled materials, a random spinorbit coupling can be achieved when hopping (hybridization) between two orbitals with opposite parity acquires random spatial modulation. Yet another CSB but vectorlike type of disorder is a random axial Zeeman coupling. Its source is the different $g$ factor of two hybridizing bands that touch at the Weyl point [68-70]. Therefore, when magnetic impurities are injected in the system, such disorder is naturally introduced, and depending on the relative strength of the $g$ factor in different bands, one can access regular (intranode) or axial (internode) random magnetic coupling. Finally, two different types of CSB mass disorders that tend to gap out the Weyl points are represented by random charge- or spindensity-wave order, depending on the microscopic details [71]. These disorders correspond to random scalar and pseudoscalar mass in the field-theory language. Because of their presence, Weyl nodes are gapped out in each disorder configuration, but the sign of the gap is random from realization to realization, and in the thermodynamic limit the nodes remain gapless. To the best of our knowledge, it is currently unknown how to tune the strength of all individual sources of elastic scattering in real Weyl materials. Nevertheless, we elucidate how all possible disorders can be obtained from a simple effective tight-binding model on a cubic lattice for a WSM with two nodes (see Appendix D), allowing us to numerically investigate the effects of generic disorder in this system.

Here we address the stability of a disordered WSM (i) in the field-theoretical framework by using two different RG schemes: (a) an $\epsilon_{m}$ expansion about a critical disorder distribution, where $\epsilon_{m}=1-m$, with the Gaussian white noise distribution realized as $m \rightarrow 0$, and (b) $\epsilon_{d}=d-2$ expansion about $d_{l}=2$, the lower critical spatial dimension for WSM-metal QPT; and (ii) lattice-based numerical evaluation of average DOS by using the kernel polynomial method (KPM) [72] in the presence of generic chiralsymmetric disorder [see Fig. 3 (upper panel)] as well as nonchiral disorder [see Fig. 3 (lower panel)]. Comparisons between the field-theoretic predictions and numerical findings for all chiral disorders are given in Table I. Our central results can be summarized as follows.

(1) From the scaling analysis we show in Sec. IV that all types of disorder (both CSP and CSB) are irrelevant perturbations in a WSM. This outcome is also supported numerically, see Fig. 3, depicting that DOS scales as $\varrho(E) \sim|E|^{2}$ for small energy $(E)$, when generic disorder is sufficiently weak.

(2) We show in Sec. V that irrespectively of the details of two distinct $\epsilon$ expansions, in the presence of a CSP disorder, the WSM-metal QPT takes place through either a QCP (when either potential or axial potential disorder is present) or a line of QCPs (when both types of scalar disorder are present simultaneously), characterized by critical exponents,

$$
z=1+\frac{\epsilon}{2}+\mathcal{O}\left(\epsilon^{2}\right), \quad \nu^{-1}=\epsilon+\mathcal{O}\left(\epsilon^{2}\right),
$$




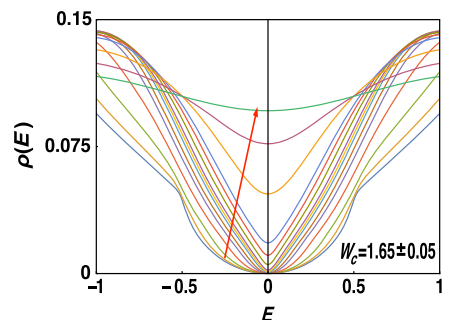

(a)

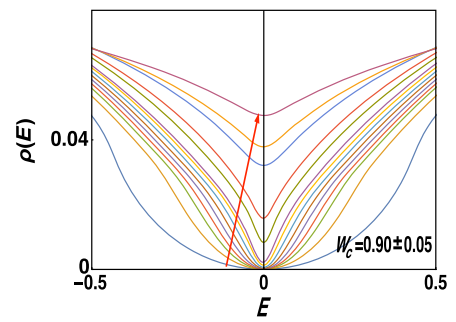

(e)

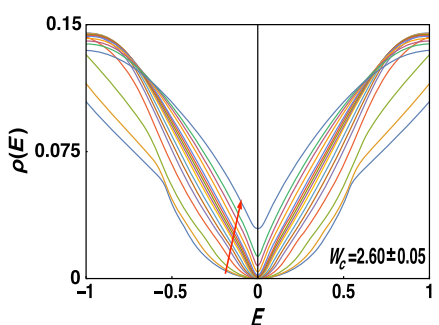

(b)

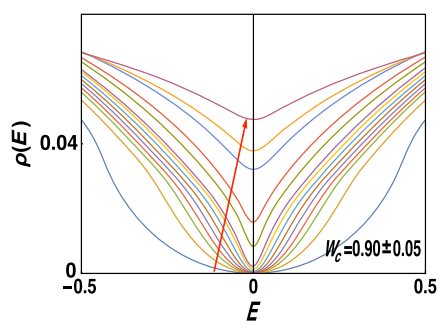

(f)

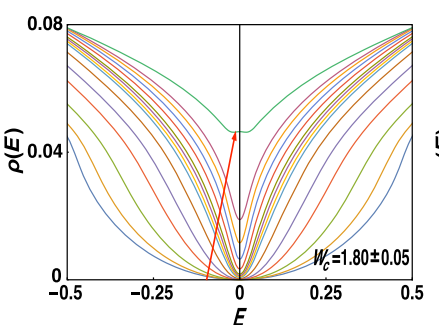

(c)

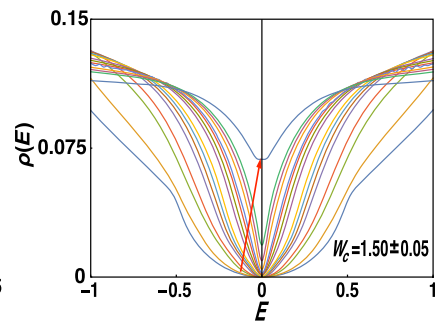

(g)

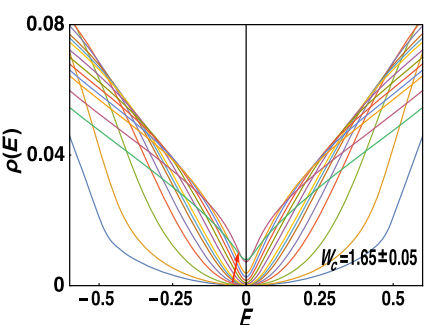

(d)

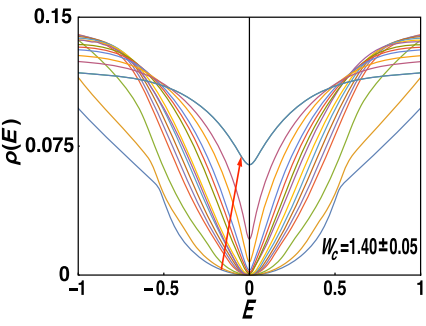

(h)

FIG. 3. Scaling of numerically evaluated (using the kernel polynomial method [72]) average density of states in dirty Weyl semimetals in the presence of (a) potential, (b) axial, (c) axial current, (d) current, (e) spin-orbit (represented by temporal component of tensor), (f) axial magnetic (represented by spatial component of tensor), (g) scalar mass, and (h) pseudoscalar mass disorder for weak to strong disorder regime, in a cubic lattice of linear dimension $L=220$ (see Table III for definition and field-theoretic nomenclature). Notice that for weak enough disorder, ADOS $\varrho(E) \sim|E|^{2}$ for $|E| \ll 1$. In the metallic phase, appearing for strong enough disorder, ADOS at zero energy $\varrho(0)$ becomes finite. Around a (nonuniversal) critical strength of disorder $W=W_{c}$ the ADOS scales as $\varrho(E) \sim|E|$ for $|E| \ll 1$. Since $\varrho(E) \sim|E|^{d / z-1}$, the dynamic scaling exponent $z \approx 1.5$ across the WSM-metal quantum phase transitions, irrespective of the nature of the elastic scatterers. Disorders in (a)-(d) preserve the emergent global chiral symmetry and represent intranode scattering, while the remaining ones [(e)-(h)] break that symmetry and represent internode scattering. Numerically extracted critical exponents across WSM-metal QPTs and their comparison with the field-theoretic predictions are displayed in Tables I and II, suggesting an excellent agreement between these two methods and emergence of a superuniversality across WSM-metal QPT. The strength of disorder increases monotonically in the direction of the red arrow in each panel.

obtained from the leading order in $\epsilon$ expansions, where $\epsilon=\epsilon_{m}$ or $\epsilon_{d}$, and $\epsilon=1$ corresponds to the physical situation. Therefore, irrespective of the nature of elastic scatterers, the universality class of the WSM-metal QPT in the presence of a CSP disorder is unique, and we name such a universality class chiral superuniversality. Even though the exponents $\nu$ and $z$ can receive higher-order corrections $\mathcal{O}\left(\epsilon^{2}\right)$, presently there is no controlled way to compute them beyond leading order in $\epsilon[40,45]$.

(3) In Sec. VI, we carry out a thorough numerical analysis of DOS in the presence of all four CSP disorders, obtained by using KPM from a lattice model [see Figs. 3(a)-3(d)]. Within the numerical accuracy we find that $z \approx 1.5$ and $\nu \approx 1$ across possible CSP disorder-driven WSM-metal QPTs (see Fig. 13 and Table I). Thus, numerically extracted values of critical exponents are in excellent agreement with the fieldtheoretic predictions from leading-order $\epsilon$ expansions, and strongly support the proposed scenario of emergent chiral superuniversality.

(4) In Sec. VII, we show that the CSB disorder can also drive a WSM-metal QPT through either an isolated QCP or a line of QCPs. Irrespective of the actual details of an $\epsilon$-expansion scheme, the values of the critical exponents at such QCP or line of QCPs are in stark contrast to the ones reported in Eq. (1), and typically $z>d$. In particular, the DSE varies continuously across the line of QCPs supported by a strong CSB disorder. On the other hand, $\nu^{-1}=\epsilon$ to the leading order in an $\epsilon$ expansion, irrespective of the RG scheme.

(5) Since $z>d$ (always), the CSP disorder as well as the higher gradient terms (inevitably present in a lattice model) become relevant at the CSB disorder-driven QCPs separating a WSM from a metallic phase. Consequently, in lattice-based simulations the WSM-metal QPT is expected to ultimately be controlled by the QCPs associated with CSP disorder. We anchor this outcome by numerically computing the DOS in the presence of all four internode scatterings (see Fig. 3 (lower panel)] and find that across WSM-metal QPTs, driven by any CSB disorder, $z \approx 1.5$ and $\nu \approx 1$ [see Table II]. Therefore, generic disorder-driven WSM-metal QPT offers a rather sparse example of superuniversality, characterized by the critical exponents $z=$ $3 / 2$ and $\nu=1$, to the leading order in $\epsilon$ expansions, which are in a reasonable good agreement with numerical findings (within error bars); see Eq. (1). 
TABLE I. Comparison of numerically extracted values of dynamic scaling exponent $(z)$ and correlation length exponent $(\nu)$ across the WSM-metal QPT (takes place at $W=W_{c}$ ), with the ones obtained from the leading-order $\epsilon$ expansions using fieldtheoretic techniques. All four disorders preserve continuous global chiral symmetry of a WSM. This comparison strongly suggests that a WSM-metal transition driven by a CSP disorder is insensitive to the nature of elastic scatterers, thus motivating an emergent chiral superuniversality class of the QPTs, cf. Sec. V. The fact that $z \approx 1.5$ for all types of disorder reflects through almost linear scaling of DOS around the WSM-metal QPT; see Fig. 3 (top panel). Here, error bars in $z$ and $\nu$ are "fitting error bars" (see Fig. 13). For detailed discussion, see Appendix E and Table IV.

\begin{tabular}{lcccccc}
\hline \hline & \multicolumn{3}{c}{ Numerical analysis } & & \multicolumn{2}{c}{ Field theory } \\
\cline { 2 - 4 } Disorder & $W_{c}$ & $z$ & $\nu$ & $z$ & $\nu$ \\
\hline Potential & $1.65 \pm 0.05$ & $1.47 \pm 0.05$ & $1.00 \pm 0.08$ & & $3 / 2$ & 1 \\
Axial & $2.60 \pm 0.05$ & $1.47 \pm 0.05$ & $1.06 \pm 0.10$ & & $3 / 2$ & 1 \\
Magnetic & $1.80 \pm 0.05$ & $1.51 \pm 0.05$ & $1.03 \pm 0.10$ & $3 / 2$ & 1 \\
Current & $1.65 \pm 0.05$ & $1.48 \pm 0.05$ & $1.02 \pm 0.09$ & $3 / 2$ & 1 \\
\hline \hline
\end{tabular}

(6) In Sec. VIII, we show that various experimentally measurable quantities, such as average DOS, dynamic conductivity, specific heat, and Grüneisen ratio, exhibit distinct scaling behavior in terms of CLE and DSE in different phases of a dirty WSM. As such, they may be useful to distinguish types of disorder in a WSM. Most importantly, distinct scaling of observables can allow one to pin the onset of various phases in real materials.

We point out that the notion of superuniversality is realized rather sparsely in condensed matter systems. Most prominent examples in this regard include the quantum Hall plateau transitions [73-75] and one-dimensional disordered superconducting wires [76]. Therefore, dirty Weyl semimetal represents, to the best of our knowledge, the only example of a three-dimensional system exhibiting superuniversality.

It is worth mentioning that for sufficiently strong disorder the metallic phase in a Weyl system undergoes a second continuous QPT into an Anderson insulating phase [28,54,77], across the red dashed line shown in Fig. 1. In Sec. IX, we address the metal-insulator Anderson transition (AT), but only in the presence of random charge impurities. Our central achievements regarding the fate of the AT in strongly disordered Weyl metal are the following.

(1) We show that a Weyl metal undergoes a second transition at stronger disorder into an Anderson insulator (AI) phase. By numerically computing the typical density of states (TDOS) at zero energy $\left[\varrho_{t}(0)\right]$, we show that $\varrho_{t}(0)$ vanishes smoothly across the Weyl metal-AI QPT, while displaying critical and single-paramter scaling. In particular, $\varrho_{t}(0)$ is pinned at zero in the WSM and AI phases, while it is finite inside the entire metallic phase. By contrast,
TABLE II. Numerically extracted critical strength of disorder for WSM-metal QPT $\left(W_{c}\right)$, dynamic scaling exponent $(z)$, and correlation length exponent $(\nu)$ in the presence of four individual disorder potentials that mix two Weyl nodes (nonchiral disorder), obtained from the scaling of average DOS. The fact that $z \approx 1.5$ for all types of disorder reflects through almost linear scaling of DOS around the WSM-metal QPT; see Fig. 3 (lower panel). For fieldtheoretic analysis of internode scatterers or nonchiral disorder, see Sec. VII. Here, error bars in $z$ and $\nu$ are fitting error bars (see Fig. 14). For detailed discussion, see Appendix E and Table IV.

\begin{tabular}{lccc}
\hline \hline Disorder & $W_{c}$ & $z$ & $\nu$ \\
\hline Spin-orbit & $0.90 \pm 0.05$ & $1.53 \pm 0.05$ & $1.01 \pm 0.10$ \\
Axial magnetic & $0.90 \pm 0.05$ & $1.53 \pm 0.05$ & $0.99 \pm 0.12$ \\
Scalar mass & $1.50 \pm 0.05$ & $1.49 \pm 0.05$ & $0.99 \pm 0.12$ \\
Pseudoscalar mass & $1.40 \pm 0.05$ & $1.49 \pm 0.05$ & $1.01 \pm 0.11$ \\
\hline \hline
\end{tabular}

the average DOS at zero energy $[\varrho(0)]$ remains finite in the metallic as well as AI phases, while being zero only in the weakly disordered WSM. Otherwise, $\varrho(0)$ decreases smoothly and monotonically across the Weyl metal-AI QCP.

(2) We demonstrate that TDOS at zero energy displays single-parameter scaling across both (a) WSM-metal and (b) metal-AI QPTs. Specifically, the orderparameter exponent for $\varrho_{t}(0), \beta_{t}$, defined as $\varrho_{t}(0) \sim$ $|\delta|^{\beta_{t}}$, where $\delta$ defines the reduced distance from transition point, is $\beta_{t}=1.80 \pm 0.20$ across the WSM-metal QPT (which is different from the one for the average DOS at zero energy for which $\beta_{a}=1.50 \pm 0.05$ ).

(3) We show that inside the metallic phase the mobility edge, separating the localized states from the extended ones, reside at finite energy. With increasing strength of disorder the mobility edge slides down to smaller energy and across the AT the entire energy widow is occupied by localized states.

The rest of the paper is organized as follows. In Sec. II, we introduce a simple tight-binding model for a Weyl system and discuss possible phases and the phase transitions in the clean limit. In Sec. III, we demonstrate the effects of generic disorder near the clean WSM-insulator QCP and perturbatively address the effects of strong disorder. In Sec. IV, we set up the theoretical framework for addressing the role of randomness deep inside the WSM phase and introduce the notion of $\epsilon_{m}$ and $\epsilon_{d}$ expansions for perturbative treatment of disorder. This section is rather technical and readers familiar with the formalism or interested in physical outcomes may wish to skip it. We devote Sec. V to the effects of CSP disorder and promote the notion of chiral superuniversality. Detailed numerical analysis of the scaling of DOS is presented in Sec. VI. Effects of CSB disorder are discussed in Sec VII, and scaling of various physical observables, such as DOS, specific heat, conductivity, etc., across the WSM-metal 
QPT is discussed in Sec. VIII. We discuss the Anderson transition of the metallic phase at stronger disorder in Sec. IX. Concluding remarks and a summary of our main findings are presented in Sec. X. Some additional technical details are relegated to the Appendixes.

\section{LATTICE MODEL FOR WEYL SYSTEM}

Let us begin the discussion with a lattice realization of chiral Weyl fermions in a three-dimensional cubic lattice. Even though in most of the commonly known Weyl materials, such as the binary alloys TaAs and NbP, Weyl fermions emerge from complex band structures in noncentrosymmetric lattices, their salient features can be captured from a simple tight-binding model:

$$
H=\sum_{k} \psi_{k}^{\dagger}[\boldsymbol{N}(\boldsymbol{k}) \cdot \boldsymbol{\sigma}] \psi_{\boldsymbol{k}} .
$$

The two-component spinor is defined as $\psi_{k}^{\top}=\left(c_{k, \uparrow}, c_{k, \downarrow}\right)$, where $c_{k, s}$ is the fermionic annihilation operator with momentum $\boldsymbol{k}$ and spin or pseudospin projection $s=\uparrow, \downarrow$, and $\boldsymbol{\sigma}$ 's are standard Pauli matrices. We here choose

$$
N_{3}(\boldsymbol{k})=t_{z} \cos \left(k_{z} a\right)-m_{z}+t_{0}\left[2-\cos \left(k_{x} a\right)-\cos \left(k_{y} a\right)\right],
$$

where $a$ is the lattice spacing. The first term gives rise to two isolated Weyl nodes along the $k_{z}$ axis at $k_{z}= \pm k_{z}^{0}$, where

$$
\cos \left(k_{z}^{0} a\right)=\frac{t_{0}}{t_{z}}\left(\frac{m_{z}}{t_{0}}+\cos \left(k_{x} a\right)+\cos \left(k_{y} a\right)-2\right),
$$

with the following choice of pseudospin vectors:

$$
N_{1}(\boldsymbol{k})=t \sin \left(k_{x} a\right), \quad N_{2}(\boldsymbol{k})=t \sin \left(k_{y} a\right) .
$$

The second term in Eq. (3), namely, $N_{3}^{M}(\boldsymbol{k})=$ $t_{0}\left[2-\cos \left(k_{x} a\right)-\cos \left(k_{y} a\right)\right]$, plays the role of a momentum-dependent Wilson mass [58,59]. The resulting phase diagram of the above tight-binding model is displayed in Fig. 4. We subscribe to this tight-binding model in Secs. III B, VI, and IX to numerically study the effects of randomness in various regimes of a dirty Weyl system.

For the sake of simplicity, we hereafter consider only the parameter regime $-t_{0}<m_{z}<t_{0}$ and $t_{z} \leq t_{0}$, so that only a single pair of Weyl fermions is realized at $\mathbf{k}^{0}=(0,0$, $\left.\pm \cos ^{-1}\left|m_{z} / t_{z}\right|\right)$. In the vicinity of these two points the Weyl quasiparticles can be identified as left and right chiral fermions, respectively. A WSM can be found when $\left|m_{z} / t\right| \leq 1$ and the system becomes an insulator for $\left|m_{z} / t\right|>1$. Even though we here restrict our analysis within the aforementioned parameter regime, this analysis can be generalized to study the semimetal-insulator QPTs in various other regimes shown in Fig. 4.

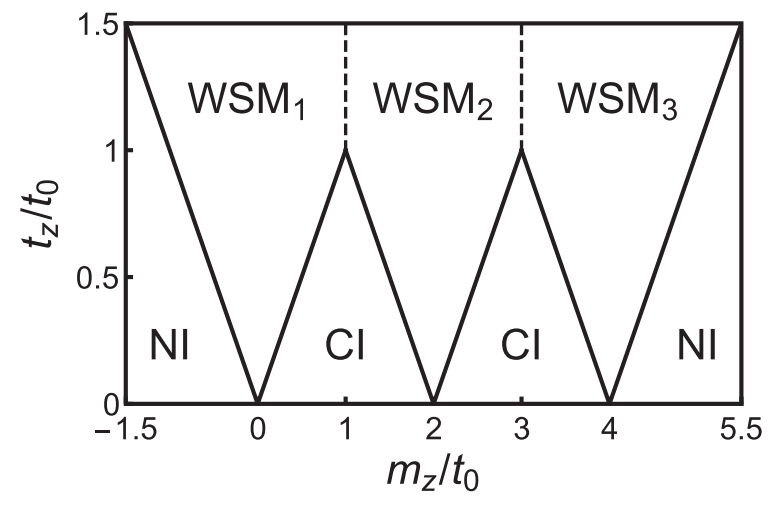

FIG. 4. The phase diagram of the clean noninteracting tightbinding model defined through Eqs. (5) and (3). Here, NI and CI, respectively, represent trivial (normal) and Chern insulators. Weyl nodes in the WSM phase are always located along the $k_{z}$ direction. Respectively, $\mathrm{WSM}_{1,2,3}$ supports one, two, and one pair of Weyl nodes. The projection of the Weyl nodes on the $x y$ plane in these phases are at the $(0,0)$ point, $(0, \pi)$ and $(\pi, 0)$ points, and $(\pi, \pi)$ point. This model therefore supports translationally active topological phases $[9,78]$. The transitions between the WSM and insulating phases (solid lines) and the ones between two distinct WSM phases (dashed lines) are continuous. We emphasize that there is no symmetry distinction among these phases.

Within this parameter regime, to capture the Weyl semimetal-insulator QPT, which occurs along the line $t_{z} / m_{z}=1$, we expand the tight-binding model around the $\Gamma=(0,0,0)$ point of the Brillouin zone to arrive at the effective low-energy Hamiltonian

$$
\hat{H}_{Q}(\Delta)=v\left(\sigma_{1} k_{x}+\sigma_{2} k_{y}\right)+\sigma_{3}\left(b k_{z}^{2}-\Delta\right),
$$

where $v=t a$ is the Fermi velocity in the $x y$ plane and $b=t_{z} a^{2} / 2$ bears the dimension of inverse mass. For $\Delta=t_{z}-m_{z}<0$, the system becomes an insulator (Chern or trivial). On the other hand, when $\Delta>0$, the lattice model describes a WSM. The QPT in this clean model between these two phases takes place at $\Delta=0$. Hence, $\Delta$ plays the role of a tuning parameter across the WSM-insulator QPT. The QCP separating these two phases is described by an anisotropic semimetal, captured by the Hamiltonian $H_{Q}(0)$ in Eq. (6), that in turn also determines the universality class of the transition. Notice that the expansion of the lattice Hamiltonian [see Eq. (5)] also yields terms $\sim k_{x}^{2}$ and $\sim k_{y}^{2}$ and higher order (from the Wilson mass), which are, however, irrelevant in the RG sense, and therefore do not affect the critical theory for the WSM-insulator QPT. Hence, we omit these higher gradient terms for now. We discuss the paramount importance of such higher gradient terms close to the CSB disorder-driven WSM-metal QPT in Sec. VII. Next, we address the stability of this quantum-critical semimetal against disorder in the system using scaling theory and RG analysis. 


\section{EFFECTS OF DISORDER ON SEMIMETAL-INSULATOR TRANSITION}

The imaginary time $(\tau)$ action associated with the lowenergy Hamiltonian [see Eq. (6)] reads as

$S_{0}=\int d \tau d^{2} x_{\perp} d x_{3} \psi^{\dagger}\left[\partial_{\tau}-i v \partial_{j} \sigma_{j}-\sigma_{3}\left(b \partial_{3}^{2}+\Delta\right)\right] \psi$.

In proximity to the Weyl semimetal-insulator QPT, the system can be susceptible to both random charge and random magnetic impurities, and their effect can be captured by the Euclidean action,

$$
\begin{aligned}
S_{D}= & \int d \tau d^{2} x_{\perp} d x_{3} \psi^{\dagger}\left[V_{0}(\boldsymbol{x}) \sigma_{0}+V_{\perp}(\boldsymbol{x})\left(\sigma_{1}+\sigma_{2}\right)\right. \\
& \left.+V_{z}(\boldsymbol{x}) \sigma_{3}\right] \psi
\end{aligned}
$$

where $V_{j}(\boldsymbol{x})$ are random variables. The effect of random charge impurities is captured by $V_{0}(\boldsymbol{x})$, while $V_{\perp}(\boldsymbol{x})$ and $V_{z}(\boldsymbol{x})$ represents random magnetic impurities with the magnetic moment residing in the easy or $x y$ plane and in the $z$ direction (denoted here by $x_{3}$ for notational clarity), respectively, which we allow due to the anisotropy of the Hamiltonian [see Eq. (6)]. All types of disorder are assumed to be characterized by Gaussian white noise distributions.

The scale invariance of the noninteracting action [see Eq. (7)] mandates the following scaling ansatz: $\tau \rightarrow e^{l} \tau$, $(x, y) \rightarrow e^{l}(x, y)$, and $x_{3} \rightarrow e^{l / 2} x_{3}$, followed by the rescaling of the field operator $\psi \rightarrow e^{-5 l / 4} \psi$, where $l$ is the logarithm of running RG scale. The scaling dimension of the tuning parameter $\Delta$ is then given by $[\Delta]=1$, implying that $\Delta$ is a relevant perturbation at the WSMinsulator QCP, located at $\Delta=0$. The scaling dimension of the tuning parameter $\Delta$ plays the role of the correlation length exponent $(\nu)$ at this QCP, implying $\nu=1$. In the presence of disorder, as we show in Appendix A, the Harris stability criterion [27] can be generalized for the WSMinsulator QCP with the quantum-critical theory of the form given by Eq. (6), but in a system with the topological or monopole charge $c$ [see Eq. (A1)]. The generalized Harris criterion then suggests that WSM-insulator QCP in a clean system remains stable against sufficiently weak disorder only if

$$
\nu>\frac{2}{d_{*}}, \quad \text { with } \quad \frac{2}{d_{*}}=\frac{4 c}{(4+c)},
$$

and $d_{*}$ as the effective spatial dimensionality of the system under the coarse-graining procedure. At the WSM-insulator QCP $\nu=1$, and the critical excitations residing at $\Delta=0$ are therefore stable against weak disorder when $c=1$ [regular WSM; see Eq. (6)]. We next analyze the effects of disorder on the WSM-insulator QCP using a RG approach. The same outcome can be arrived at from the computation of inverse scattering lifetime $(1 / \tau)$ within the framework of self-consistent Born approximation (see Appendix J).

\section{A. Perturbative RG analysis}

After performing the disorder averaging in the action [see Eq. (8)] within the replica formalism, we arrive at the replicated Euclidean action,

$$
\begin{aligned}
\bar{S}= & \int d \tau d^{2} x_{\perp} d x_{3} \psi_{a}^{\dagger}\left\{\partial_{\tau}-i v\left(\partial_{x} \sigma_{1}+\partial_{y} \sigma_{2}\right)+\sigma_{3}\left[(-i)^{n} b_{n} \partial_{3}^{n}-\Delta\right]\right\} \psi_{a}-\int d \tau d \tau^{\prime} d^{2} x_{\perp} d x_{3}\left(\frac{\Delta_{0}}{2}\left(\psi_{a}^{\dagger} \psi_{a}\right)_{(\boldsymbol{x}, \tau)}\left(\psi_{b}^{\dagger} \psi_{b}\right)_{\left(\boldsymbol{x}, \tau^{\prime}\right)}\right. \\
& \left.+\frac{\Delta_{\perp}}{2} \sum_{j=1,2}\left(\psi_{a}^{\dagger} \sigma_{j} \psi_{a}\right)_{(\boldsymbol{x}, \tau)}\left(\psi_{b}^{\dagger} \sigma_{j} \psi_{b}\right)_{\left(\boldsymbol{x}, \tau^{\prime}\right)}+\frac{\Delta_{z}}{2}\left(\psi_{a}^{\dagger} \sigma_{3} \psi_{a}\right)_{(\boldsymbol{x}, \tau)}\left(\psi_{b}^{\dagger} \sigma_{3} \psi_{b}\right)_{\left(\boldsymbol{x}, \tau^{\prime}\right)}\right),
\end{aligned}
$$

where $a, b$ are replica indices. Notice that here we have replaced $k_{3}^{2} \rightarrow k_{3}^{n}$, with $n$ as an even integer so that such deformation of spectrum does not change the symmetry of the system. We will show that such deformation of the quasiparticle spectrum allows us to control the perturbative RG calculation in terms of disorder coupling. The above imaginary-time action $(\bar{S})$ remains invariant under the space-time scaling $(x, y) \rightarrow e^{l}(x, y), x_{3} \rightarrow e^{l / n} x_{3}$, and $\tau \rightarrow e^{z l} \tau$. At the bare level the scale invariance of the free part of the action requires the field-renormalization factor $Z_{\psi}=e^{-(2+1 / n) l}$ and $\psi \rightarrow Z_{\psi}^{-1 / 2} \psi$. From this scaling analysis we immediately find that the scaling dimension of disorder couplings is $\left[\Delta_{j}\right]=-1 / n$, for $j=0, \perp, z$. Therefore, at the WSMinsulator QCP, characterized by $n=2$, disorder is an irrelevant perturbation, in accordance with the prediction from the generalized Harris criterion, implying the stability of this QCP against sufficiently weak randomness. Note that disorder couplings are marginal in a hypothetical limit $n \rightarrow \infty$, for which the system effectively becomes a two-dimensional Weyl semimetal. Therefore, perturbative analysis in the presence of generic disorder is controlled via an $\epsilon_{n}$ expansion, where $\epsilon_{n}=1 / n$, about $n \rightarrow \infty$, following the spirit of $\epsilon$ expansions about upper or lower critical dimension [79] and infinite monopole charge [80,81].

Upon integrating out the fast Fourier modes within the momentum shell $\Lambda e^{-l}<k_{\perp}<\Lambda$, where $k_{\perp}=\sqrt{k_{x}^{2}+k_{y}^{2}}$, $0<k_{3}^{2}<\infty$, and accounting for pertubative corrections to one-loop order (see Fig. 5), we arrive at the following flow equations: 


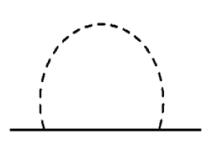

(a)

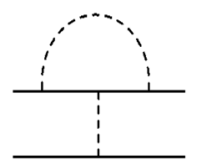

(b)

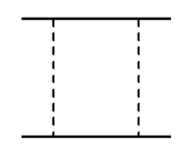

(c)

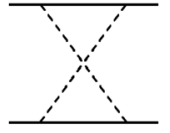

(d)
FIG. 5. One-loop diagrams contributing to the self-energy correction (a), and renormalization of disorder coupling (b)-(d). Notice that contributions from (c) ladder and (d) crossing diagram are ultraviolet divergent only in $\epsilon_{n}$ (Sec. III A) and $\epsilon_{d}$ (Sec. IV C) expansions, while they produce ultraviolet finite contribution in $\epsilon_{m}$ expansion (Sec. IV B). Here, solid (dashed) lines represent fermionic (disorder) field.

$\beta_{X}=-X\left(\Delta_{0}+2 \Delta_{\perp}+\Delta_{z}\right)=(1-z) X$,

$\beta_{\Delta}=\Delta\left[1+\Delta_{0}-2 \Delta_{\perp}+\Delta_{z}\right]$,

$\beta_{\Delta_{0}}=-\epsilon_{n} \Delta_{0}+2 \Delta_{0}\left(\Delta_{0}+2 \Delta_{\perp}+\Delta_{z}\right)$,

$\beta_{\Delta_{\perp}}=-\epsilon_{n} \Delta_{\perp}+2 \Delta_{0} \Delta_{z}$,

$\beta_{\Delta_{z}}=-\epsilon_{n} \Delta_{z}+2 \Delta_{z}\left(2 \Delta_{\perp}-\Delta_{0}-\Delta_{z}\right)+4 \Delta_{0} \Delta_{\perp}$,

in terms of dimensionless parameters,

$$
\hat{\Delta}=\frac{\Delta}{v \Lambda}, \quad \hat{\Delta}_{j}=\Delta_{j}\left(\frac{\Lambda^{\epsilon_{n}}}{(2 \pi)^{2} b_{n}^{\epsilon_{n}} v^{2-\epsilon_{n}}}\right),
$$

for $X=v, b_{n}, j=0, \perp, z, \beta_{Q} \equiv d Q / d l$ is the $\beta$ function for the running parameter $Q$, and for brevity we omit the hat notation in Eq. (11). In the above flow equations, we have kept only the leading divergent contribution that survives as $n \rightarrow \infty$. Inclusion of subleading divergences yields only nonuniversal corrections, as shown in Appendix B. The $\beta$ function for in-plane Fermi velocity $(v)$ and $b_{n}$ leads to a scale-dependent DSE:

$$
z(l)=1+\left(\Delta_{0}+2 \Delta_{\perp}+\Delta_{z}\right)(l) .
$$

Note that in this formalism the random charge impurities do not generate any new disorder, allowing us to depict the RG flow in the $\left(\Delta, \Delta_{0}\right)$ plane, as shown in Fig. 6(a).

The coupled RG flow equations [Eq. (11)] support only two fixed points.

(1) $\left(\Delta, \Delta_{0}, \Delta_{\perp}, \Delta_{z}\right)=(0,0,0,0)$, which has only one unstable direction along the $\Delta$ direction that serves as the tuning parameter for WSM-insulator QPT. This fixed point stands as a QCP in the fourdimensional coupling constant space. The correlation length exponent at this QCP is $\nu^{-1}=1$. All disorder couplings are irrelevant perturbations at this QCP [see the blue dot in Fig. 6(a)].

(2) $\left(\Delta, \Delta_{0}, \Delta_{\perp}, \Delta_{z}\right) \approx\left(0, \epsilon_{n} / 2,0,0\right)$ stands as a multicritical point with two unstable directions. At this MCP the WSM, an insulator, and the metallic phase meet. Two correlation-length exponents are $\nu_{M}^{-1}=\epsilon_{n}$ determining the relevance of disorder coupling $\Delta_{0}$,

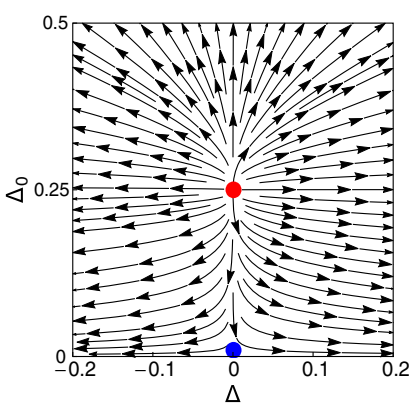

(a)

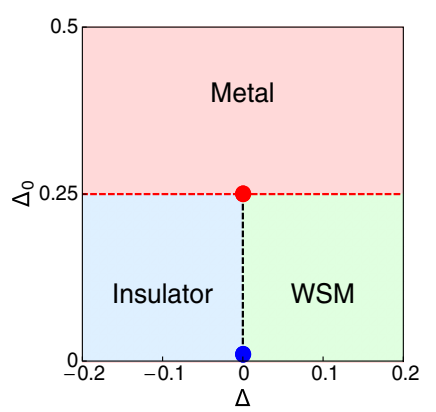

(b)
FIG. 6. (a) The RG flow diagram obtained from Eq. (11) and (b) the resulting phase diagram in the $\Delta-\Delta_{0}$ plane, for $\epsilon_{n}=1 / 2$. Here, $\Delta$ is the tuning parameter for WSM-insulator transition [see Eq. (6)], and $\Delta_{0}$ is the strength of random charge impurities. The blue dot and red dot, respectively, represent a critical and a multicritical point. The metallicity sets in through the multicritical point.

which drives the anisotropic critical semimetal [described by $\hat{H}_{Q}(0)$ ] into a diffusive metallic phase, and $\nu^{-1}=1$ that determines the relevance of the tuning parameter $\Delta$, controlling the WSM-insulator transition. The DSE for critical semimmetal-metal QPT is $z=1+\left(\epsilon_{n} / 2\right)+\mathcal{O}\left(\epsilon_{n}^{2}\right)$. Therefore, for a three-dimensional anisotropic critical semimetalmetal QPT, setting $\epsilon_{n}=1 / 2$, the critical exponents are $\nu_{M}=2$ and $z=1.25$, to the leading order in $\epsilon_{n}$ expansion.

The RG flow and the resulting phase diagrams are shown in Figs. 6(a) and 6(b), respectively. At the multicritical point the average DOS scales as $\varrho(E) \sim|E|^{d_{*} / z-1} \approx$ $|E|$ to one-loop order, since $d_{*}=5 / 2$ for $c=1$, as given by Eq. (9). Beyond the critical strength of disorder system becomes a metal where the average DOS at zero energy $[Q(0)]$ is finite and the order parameter exponent $\beta=\left(d_{*}-z\right) \nu=2.5$ determines the scaling of $\varrho(0)$ according to $\varrho(0) \sim \delta^{\beta}=\delta^{2.5}$ in the metallic phase, where $\delta=$ $\left(\Delta_{0}-\Delta_{0}^{*}\right) / \Delta_{0}^{*}$ is the reduced disorder coupling from the critical one at $\Delta_{0}=\Delta_{0}^{*}$. Next, we numerically demonstrate (a) stability of WSM-insulator QCP at weak disorder and (b) emergence of a metallic phase through a MCP at finite disorder coupling that masks the direct transition between WSM and insulator by numerically computing the average DOS using the kernel polynomial method. As a natural outcome of this exercise, we will also show that numerically extracted values of the exponents, $z$ and $\nu$, at the MCP, associated with the critical excitations-metal QPT agree with the predictions from the leading-order $\epsilon_{n}$ expansion. We also note that the same spirit of RG analysis, controlled via "band flattening", can also be applied to address the effect of randomness deep inside the WSM phase. We, however, relegate that discussion to Appendix I.

For the sake of simplicity, we here neglect quantum corrections to RG flow equations due to nontrivial 
dispersion along $k_{z}$. Nonetheless, our formal approach allows us to systematically account for such quantum corrections, controlled via another small parameter $1 / n$ (in the spirit of a $1 / N$ expansion, where $N$ counts the number of fermion flavors [79]). Therefore, our RG analysis is ultimately controlled by two small parameters $\epsilon_{n}$ (measuring the deviation from the marginality condition for disorder, i.e., two spatial dimensions, leading to nontrivial bare scaling dimension $\left[\Delta_{j}\right]=-\epsilon_{n}$ for all disorder couplings with $j=0, \perp, z$ ) and $1 / n$ [measuring the strength of the band dispersion in $k_{z}$ direction and thus controlling the quantum (loop) corrections arising from finite band curvature in this direction]. In this regard the RG analysis follows the spirit of simultaneous $\epsilon$ and $1 / N$ expansions [79]. Only at the very end of the calculation we set $\epsilon_{n}=1 / 2$ and $n=2$ (physically relevant situation). This analysis is presented in detail in Appendix B 1. The resulting exponents (after accounting for $1 / n$ quantum corrections), namely, $z=1.245$ and $\nu=2$, are sufficiently close to the ones we report here by taking $n \rightarrow \infty$ in the perturbative loop corrections.

\section{B. Scaling of density of states near WSM-insulator QCP: Numerical demonstration of the MCP}

Before we discuss the scaling behavior of the average DOS along the WSM-insulator phase boundary and inside the metallic phase, setting in through the instability of the critical semimetallic phase, let us point out some crucial subtle issues associated with such analysis. Note that the ADOS of the critical semimetal [described by $\hat{H}_{Q}(0)$ in Eq. (6)] vanishes as $Q(E) \sim|E|^{3 / 2}$, while that in the WSM phase vanishes as $\varrho(E) \sim|E|^{2}$. But, in the insulating phase, average DOS displays hard gap. Based on scaling analysis we expect WSM, insulator, and the critical semimetal to be stable against sufficiently weak disorder. We exploit these characteristic features to pin the WSM-insulator phase boundary for weak disorder. On the other hand, for stronger disorder onset of a metallic phase can be identified from the existence of finite ADOS at zero energy. Following these diagnostic tools we arrive at the phase diagram of a Weyl material residing in close proximity to the WSM-insulator QPT; see Fig. 2 (left). We are ultimately interested in exposing the existence of a MCP in the $\left(m_{z}, t_{z}\right)$ plane [the red dot in Fig. 6(a)], which has two relevant directions. One of them controls critical semimetal-metal QPT, while the other one drives WSM-insulator QPT. Since we consider the former transition, our focus will be restricted on the black dashed line shown in Fig. 2.

More specifically, we here compute the average DOS by employing the KPM [72] starting with the tight-binding model, introduced in Eqs. (2), (3), and (5), and staying in close vicinity of $m_{z} / t_{0}=0.5$ and $t_{z} / t_{0}=0.5$ (see the phase diargam in Fig. 4). The tight-binding model is implemented on a cubic lattice with periodic boundary conditions in all three directions and the linear dimensionality of the system in each direction is $L=140$. Even though average DOS is a self-averaged quantity, we perform average over 20 random disorder realizations to minimize the residual statistical fluctuations, compute 4096 Chebyshev moments, and take trace over 12 random vectors to obtain the average DOS. For the sake of simplicity we here account for only random charge impurities. Potential disorder is distributed uniformly and randomly within the range $[-W, W]$. The scaling of average DOS can be derived in the following way.

Since we are following only one relevant direction associated with the MCP, effectively it can be treated as a simple QCP across which various physical observables (such as average DOS) display single parameter scaling. Note that the total number of states $N(E, L)$ in a $d$ dimensional system of linear dimension $L$, below the energy $E$, is proportional to $L^{d}$, and in general is a function of two dimensionless parameters $L / \xi$ and $E / E_{0}$. Here, $\xi \sim$ $\delta^{-\nu}$ is the correlation length that diverges at the QCP, located at $\delta=0$, where $\delta=\left[\left(W-W_{c}\right) / W_{c}\right]$ is the reduced distance from the QCP, located at $W=W_{c}$. Consequently, the correlation energy, defined as $E_{0} \sim \delta^{\nu z}$, vanishes as the QCP is approached from either side of the transition [82]. Following the standard formalism of scaling theory, we then can write

$$
N(E, L)=(L / \xi)^{d} G\left(E / \delta^{\nu z}, L / \delta^{-\nu}\right),
$$

where $G$ is an universal but unknown scaling function. Therefore, from the definition of average DOS, $\varrho(E, L)=L^{-d} d N(E, L) / d E$, we arrive at the following scaling form:

$$
\varrho(E, L)=\delta^{\nu(d-z)} F\left(|E| \delta^{-\nu z}, \delta L^{1 / \nu}\right),
$$

where $F$ is yet another universal, but typically unknown, scaling function. However, we can access the behavior of the scaling function in different regimes along the black dashed line shown in Fig. 2 (left), which we exploit to compute critical exponents characterizing the critical semimetal-metal QPT across the MCP. In the final step we have used the fact that average DOS remains particle-hole symmetric, but on average. Note that we will use exactly the same scaling function deep inside the WSM phase in the presence of generic disorder, discussed in Sec. VI. We must stress here that in the above expression $d=d_{*}$, the effective dimensionality of the system, defined in Eq. (9), when we address the scaling of ADOS along the phase boundary between the WSM and an insulator, and across the QPT to a metallic phase through the MCP, shown in Fig. 2 (left). On the other hand, we set $d=3$ (physical dimensionality) while addressing the WSM-metal transition, since the electronic dispersion is linear and isotropic in a WSM. 


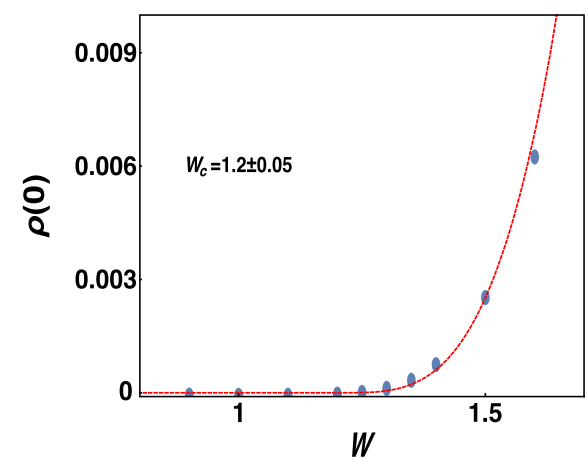

(a)

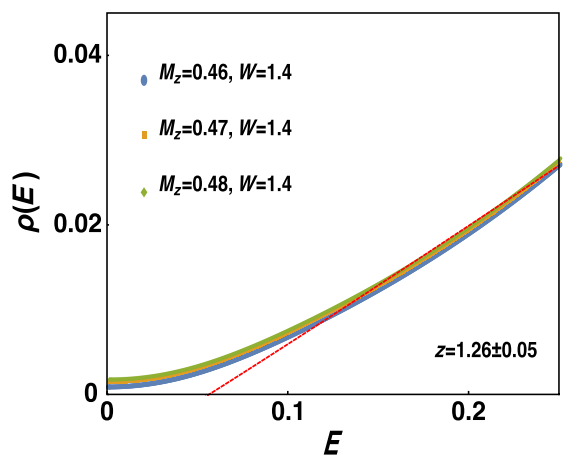

(b)

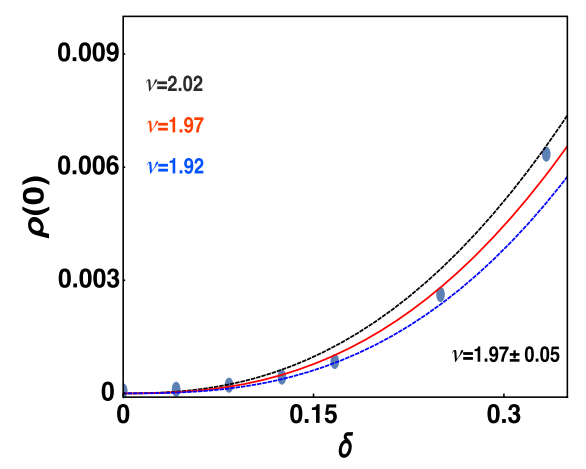

(c)

FIG. 7. Analysis of average density of states in various regimes along the black dashed line shown in Fig. 2 (left). Recall the black dashed line for weak disorder defines the phase boundary between the WSM and insulator, while when extended into the metallic phase [the red shaded regime in Fig. 2 (left)] captures the instability of critical excitations residing at the WSM-insulator QCP toward the formation of a metallic phase. (a) Scaling of average DOS at zero energy $[\varrho(0)]$ along the black dashed line as a function of increasing disorder $(W)$, showing that $\varrho(0)$ remains pinned at zero up to a critical strength of disorder, $W_{c}=1.20 \pm 0.05$. (b) Scaling of average DOS at finite energy $[\varrho(E)]$ around the multicritical point residing in the two-dimensional coupling constant space $\left(m_{z}, W\right)$, indicating the dynamic scaling exponent for critical excitation-metal QPT is $z=1.26 \pm 0.05$. (c) Scaling of $\varrho(0)$ along the black dashed line inside the metallic phase indicating that correlation length exponent for critical excitation-metal QPT is $\nu=1.97 \pm 0.05$. Details of the data analysis are presented in Sec. III B. The quoted error bars in $z$ and $\nu$ are fitting error bars. See Appendix E and Table IV (last row) for further details of data analysis.

First of all, notice that $\operatorname{ADOS} \varrho(0)$ is pinned to zero along the phase boundary between the WSM and insulator for weak enough disorder, as shown in Fig. 7(a). Therefore, critical semimetal separating these two phases remains stable against weak disorder and the nature of the WSMinsulator direct transition remains unchanged for weak enough randomness. However, beyond a critical strength of disorder, $W_{c}=1.20 \pm 0.05, \varrho(0)$ becomes finite and metallicity sets in through the MCP; see Figs. 2 (left) and 7(a). Beyond this point there exists no direct transition between the WSM and an insulator. Also note for $W \ll W_{c}$, $\varrho(E) \sim|E|^{1.5}$, as shown in Fig. 2 (right), as expected, since in the clean system $z=1$ and $d_{*}=5 / 2$.

Now we consider very close proximity to the MCP, located at $W=W_{c}$ along the disorder axis. At this MCP average DOS becomes independent of $\delta$, yielding $F(x) \sim$ $x^{d_{*} / z-1}$. By comparing $\varrho(E)$ with $E$, we obtain the DSE associated with critical semimetal-metal QPT to be $z=1.26 \pm 0.05$; see Fig. 7(b).

Next we move into the metallic phase, but continue to follow the black dashed line from Fig. 2 (left). In the metallic phase $\varrho(0)$ becomes finite [see Fig. 7(a)]. Thus to the leading order $F(x) \sim x^{0}$ and consequently $\varrho(0) \sim$ $\delta^{\left(d_{*}-z\right) \nu}$. With the prior notion of $z=1.26 \pm 0.05$, now by comparing $Q(0)$ versus $\delta$ we obtain the CLE at the MCP associated with the critical semimetal-metal QPT to be $\nu=1.97 \pm 0.05$, as shown in Fig. 7(c). [After accounting for the variation in the location of $W_{c}$ and determination of $z$, we finally obtain $\nu=1.98 \pm 0.10$; see Appendix E for discussion and Table IV (last row) for analysis.]
Therefore, numerically extracted values of two critical exponents, namely, $\nu=1.97$ and $z=1.26$, at the MCP associated with the critical semimetal-metal QPT match quite satisfactorily with the field-theoretic prediction obtained from an $\epsilon_{n}$ expansion introduced in this work, which allows us to control the RG calculation by tuning the flatness of the quasiparticle dispersion along the $k_{z}$ direction: a controlled ascent from two spatial dimensions.

We now discuss two different types of data collapse across the disorder-driven MCP. The results are shown in Fig. 8. First, we focus on the largest system with $L=220$. From Eq. (14), upon neglecting the finite-size effects, we compare $\varrho(E)|\delta|^{-\left(d_{*}-z\right) \nu}$ versus $|E||\delta|^{-\nu z}$ along the black line from Fig. 2 (left). With numerically obtained values of $\nu$ and $z$ we find that all data nicely collapse onto two branches (corresponding to the anisotropic semimetal and metallic sides of the QPT), which meet in the critical regime, as shown in Fig. 8 (left). Next, we compare the ADOS at zero energy in the metallic phase, namely, $\varrho(0) L^{d_{*}-z}$ versus $L^{1 / \nu} \delta$, in systems of different sizes $(L)$, as shown in Fig. 8 (right). We also obtain excellent finite-size data collapse for a wide range of system sizes using already numerically extracted values of $\nu$ and $z$. Therefore, field-theoretic predictions and numerical findings across the disorderdriven MCP are in good agreement with each other. Next, we address the effects of disorder inside the WSM phase by pursing complementary field-theoretic and numeric approaches. 


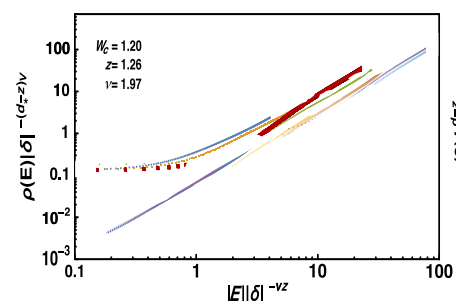

(a)

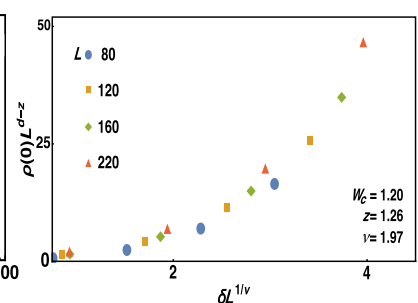

(b)
FIG. 8. (a) Collapse of average DOS at finite energy (obtained in system with $L=220$ ) across the multicritical point shown in Fig. 2 (left). All data collapse reasonably well onto two branches corresponding to anisotropic semimetal (upper branch) and metallic phase (lower branch), which tend to meet in the critical regime. (b) Data collapse of average DOS at zero energy for different system sizes inside the metallic phase, appearing across the MCP. These two data collapses are obtained with numerically extracted critical exponents $z=1.26$ and $\nu=1.97$ (see Fig. 7), with $d_{*}=5 / 2$.

Note that the MCP, where WSM, an insulator, a metal, and the critical anisotropic semimetal meet, possesses two relevant directions; see Fig. 6(a). Hence, at finite energies two quantum-critical fans associated with (1) critical anisotropic semimetal-metal and (2) WSM-metal QPTs (characterized by distinct sets of critical exponents) intertwine. Thus, obtaining a high-quality data collapse at finite energies [see Fig. 8 (left)] across this MCP is quite challenging, and qualitatively it is slightly worse than that across the WSM-metal QPT (sufficiently far from the MCP), shown in Figs. 13 and 14 (third column). Still, roughly 300 data points effectively fall on two branches [the top (bottom) one representing metallic (anisotropic semimetallic) phase] with numerically extracted mean values of the exponents, $z=1.26$ and $\nu=1.97$, in good agreement with analytical predictions from leading order in $\epsilon_{n}$ expansion $(z=1.25$ and $\nu=2)$. The quality of finitesize data collapse obtained from the scaling of $\varrho(0)$ in different systems [see Fig. 8 (right)] is yet quite comparable to the ones shown in Figs. 13 and 14 (fourth column) across the WSM-metal QPT.

\section{DIRTY WEYL SEMIMETAL: MODEL AND SCALING ANALYSIS}

In this section, we set up the field-theoretical framework to analyze the role of disorder when the system is deep inside the WSM phase. We introduce the notion of two different $\epsilon$ expansions: (a) an $\epsilon_{m}$ expansion about a critical disorder distribution, where $\epsilon_{m}=1-m$ with Gaussian white noise distribution recovered as $m \rightarrow 0$, and (b) an $\epsilon_{d}$ expansion, with Gaussian white noise distribution from the outset, about the lower critical dimension $d_{c}=2$ for WSM-metal QPT, where $\epsilon_{d}=d-2$, and therefore for three spatial dimensions $\epsilon_{d}=1$.

\section{A. Hamiltonian and action}

The effective low-energy description of WSM can be obtained by expanding the lattice Hamiltonian [see Eq. (5)] around the Weyl nodes located at $\mathbf{k}^{0}=\left(0,0, \pm k_{z}^{0}\right)$, with $k_{z}^{0}=\cos ^{-1}\left(m_{z} / t_{z}\right)$. The resulting low-energy Hamiltonian reads

$$
H_{W}=\tau_{0} \otimes v\left(k_{x} \sigma_{1}+k_{y} \sigma_{2}\right)+\tau_{3} \otimes \sigma_{3} v_{z} k_{z},
$$

where $v=t a, v_{z}=a \sqrt{t_{z}^{2}-m_{z}^{2}}$, and the momentum is measured from the Weyl nodes. For simplicity we hereafter take the Fermi velocity to be isotropic, $v=v_{z}$, so that the low-energy Hamiltonian becomes rotationally symmetric. Upon performing a unitary rotation with $U=\sigma_{0} \oplus \sigma_{3}$, the above Hamiltonian assumes a quasirelativistic form $H_{W}=i \gamma_{0} \gamma_{j} v k_{j}$, where $\gamma_{0}=\tau_{1} \otimes \sigma_{0}, \gamma_{j}=\tau_{2} \otimes \sigma_{j}$ for $j=1,2,3$ are mutually anticommuting $4 \times 4$ Hermitian matrices, and summation over repeated spatial indices is assumed. To close the Clifford algebra of five mutually anticommuting matrices, we define $\gamma_{5}=\tau_{3} \otimes \sigma_{0}$. Two sets of Pauli matrices $\sigma_{\mu}$ and $\tau_{\mu}$, respectively, operate on spin or pseudospin and valley or chiral (left and right) indices. The low-energy effective Hamiltonian enjoys a variety of emergent discrete and continuous symmetries. The above Hamiltonian is invariant under a pseudo-time-reversal symmetry, generated by an antiunitary operator $\mathcal{T}=$ $\gamma_{0} \gamma_{2} K$, where $K$ is the complex conjugation, a charge conjugation symmetry, generated by $\mathcal{C}=\gamma_{2}$, and parity or inversion symmetry generated by $\mathcal{P}=\gamma_{0}$. Furthermore, the Hamiltonian [see Eq. (15)] also possesses a global chiral $\mathrm{U}(1)$ symmetry, generated by $\gamma_{5}$, which in the lowenergy limit corresponds to the generator of translational symmetry [66].

To incorporate the effects of disorder we consider the following minimal continuum action for a dirty WSM:

$$
S=\int d^{d} \boldsymbol{x} d \tau\left[\bar{\Psi}\left(\gamma_{0} \partial_{\tau}+v \gamma_{j} \partial_{j}\right) \Psi-\varphi_{N}(\bar{\Psi} N \Psi)\right]
$$

with $\boldsymbol{x}$ as $d$-dimensional spatial coordinates, the fourcomponent spinor $\Psi^{\dagger}=\left(u_{\uparrow,+}^{\dagger}, u_{\downarrow,+}^{\dagger}, u_{\uparrow,-}^{\dagger}, u_{\downarrow,-}^{\dagger}\right)$, where $u_{\sigma, \tau}^{\dagger}$ is the fermionic creation operator near the Weyl point at $\tau \boldsymbol{k}^{0}$ for $\tau= \pm$ (left or right) and with spin $\sigma=\uparrow, \downarrow$, while $\bar{\Psi}=\Psi^{\dagger} \gamma_{0}$, as usual. Various disorder fields $\varphi_{N}$, coupled to the fermion bilinears, are realized with different choices of $4 \times 4$ matrices $N$, as shown in Table III. Notice that the matrices associated with four types of disorder anticommute with $\gamma_{5}$ and represent chiral-symmetric disorder, while for the other four types of disorder, $\left[N, \gamma_{5}\right]=0$ and the corresponding disorder vertex breaks the $\mathrm{U}(1)$ chiral symmetry. As we demonstrate in this paper, such a global chiral symmetry plays a fundamental role in classifying the disorder-driven WSM-metal QPTs. 
TABLE III. Various types of disorder represented by fermionic bilinears $(j=1,2,3)$, together with their symmetries under pseudo-time-reversal $(\mathcal{T})$, parity $(\mathcal{P})$, continuous chiral rotation $\left(U_{c}\right)$, and charge conjugation $(\mathcal{C})$. The disorder couplings are represented by $\Delta_{N}$ and $\Sigma_{\mu \nu}=\left[\gamma_{\mu}, \gamma_{\nu}\right] /(2 i)$. Note that true timereversal symmetry in WSM is already broken. The pseudo-timereversal symmetry $\mathcal{T}$ is generated by an antiunitary operator $\gamma_{0} \gamma_{2} K$, where $K$ is complex conjugation, such that $\mathcal{T}^{2}=-1$ (the true time-reversal operator is $\left.\gamma_{1} \gamma_{3} K\right)$. The parity operator is $\mathcal{P}=\gamma_{0}$, while the charge-conjugation operator is $\mathcal{C}=\gamma_{2}$. The continuous chiral symmetry $\left(U_{c}\right)$ is generated by $\gamma_{5}$, the generator of translational symmetry in the continuum limit in a clean Weyl semimetal [66]. The Hermitain $\gamma$ matrices satisfy standard anticommutation relation $\left\{\gamma_{\mu}, \gamma_{\nu}\right\}=2 \delta_{\mu \nu}$ for $\mu, \nu=0,1,2,3$, 5 , and for explicit representation of $\gamma$ matrices, see Sec. IV A. Here $\checkmark$ and $\times$ signify even and odd under a symmetry operation, respectively. With a slightly different tight-binding model, where $N_{j}(\mathbf{k})=t \cos \left(k_{j} a\right) \quad$ and $\quad N_{3}^{M}(\mathbf{k})=\left[\sin \left(k_{1} a\right)+\sin \left(k_{2} a\right)-\right.$ $2 \sin \left(k_{3} a\right)$ ] [see Eq. (2)], the axial current corresponds to magnetization, temporal and spatial tensors to spin-orbit and axial magnetization, respectively. However, such microscopic details do not alter any physical outcome.

\begin{tabular}{lcccccc}
\hline \hline Bilinear & Physical quantity & $\mathcal{T}$ & $\mathcal{P}$ & $U_{c}$ & $\mathcal{C}$ & Coupling \\
\hline $\bar{\Psi} \gamma_{0} \Psi$ & Chemical potential & $\checkmark$ & $\checkmark$ & $\checkmark$ & $x$ & $\Delta_{V}$ \\
$\bar{\Psi} \gamma_{0} \gamma_{5} \Psi$ & Axial potential & $\checkmark$ & $x$ & $\checkmark$ & $\checkmark$ & $\Delta_{A}$ \\
$\bar{\Psi} \Psi$ & Scalar mass & $\boldsymbol{x}$ & $\checkmark$ & $x$ & $\checkmark$ & $\Delta_{S}$ \\
$\bar{\Psi} i \gamma_{5} \Psi$ & Pseudoscalar mass & $\checkmark$ & $\boldsymbol{x}$ & $\boldsymbol{x}$ & $\checkmark$ & $\Delta_{P S}$ \\
$\bar{\Psi} i \gamma_{5} \gamma_{j} \Psi$ & Axial current & $\boldsymbol{x}$ & $\checkmark$ & $\checkmark$ & $\checkmark$ & $\Delta_{M}$ \\
$\bar{\Psi} i \gamma_{j} \Psi$ & Current & $\boldsymbol{x}$ & $\boldsymbol{x}$ & $\checkmark$ & $\boldsymbol{x}$ & $\Delta_{C}$ \\
$\bar{\Psi} i \Sigma_{0 j} \Psi$ & Temporal tensor & $\boldsymbol{x}$ & $\boldsymbol{x}$ & $\boldsymbol{x}$ & $\boldsymbol{x}$ & $\Delta_{S O}$ \\
$\bar{\Psi} \Sigma_{j k} \Psi$ & Spatial tensor & $\checkmark$ & $\checkmark$ & $\boldsymbol{x}$ & $\boldsymbol{x}$ & $\Delta_{A M}$ \\
\hline \hline
\end{tabular}

\section{B. $\epsilon_{m}$ expansion in three dimensions}

We assume that the disorder field obeys the distribution $[38,83]$

$$
\left\langle\varphi_{N}(\mathbf{x}) \varphi_{N}(\mathbf{y})\right\rangle=\Delta_{N} \frac{1}{|\mathbf{x}-\mathbf{y}|^{d-m}},
$$

or in the momentum space,

$$
\left\langle\varphi_{N}(\mathbf{q}) \varphi_{N}(\mathbf{0})\right\rangle=\tilde{\Delta}_{N} \frac{1}{|\mathbf{q}|^{m}},
$$

and the limit $m \rightarrow 0$ corresponds to the Gaussian white noise distribution, which we are ultimately interested in. This form of the white noise distribution stems from the following representation of the $d$-dimensional $\delta$ function [44]:

$$
\delta^{(d)}(\mathbf{x}-\mathbf{y})=\lim _{m \rightarrow 0} \frac{\Gamma\left(\frac{d-m}{2}\right)}{2^{m} \pi^{d / 2} \Gamma(m / 2)} \frac{1}{|\mathbf{x}-\mathbf{y}|^{d-m}} .
$$

We now carry out the scaling analysis of the continuum action for a WSM given by Eq. (16). The scaling dimensions of the momentum and frequency are $[q]=1$ and $[\omega]=z$. The form of the Euclidean action [see Eq. (16)] then implies that the engineering scaling dimension of the fermionic field $[\Psi]=d / 2$ and $[v]=z-1$, while the scaling dimension of the disorder field is $\left[\varphi_{N}\right]=$ $z+\eta_{\varphi_{N}}$, since the engineering dimension of the disorder field is equal to the DSE $z$ for any choice of $N$, and $\eta_{\varphi_{N}}$ is its anomalous dimension. Equation (17) then yields

$$
\left[\Delta_{N}\right]=2\left(z+\eta_{\varphi_{N}}\right)-d+m
$$

Because of linearly dispersing low-energy quasiparticles, a WSM corresponds to $z=1$ fixed point, and in $d=3$ the engineering dimension of the disorder strength is $\left[\Delta_{N}\right]=m-1$. A first implication of this result is that the white noise disorder, $m=0$, is irrelevant close to the WSM ground state in $d=3$. Second, for $m=1$, the disorder is marginal and we use that to introduce the deviation from this value as an expansion parameter $\epsilon_{m}=1-m$.

The $\beta$ function (infrared) for the disorder coupling $\Delta_{N}$ in the $\epsilon_{m}$ expansion is given in terms of its scaling dimension in Eq. (20), yielding

$$
\beta_{\Delta_{N}}=\Delta_{N}\left[-\epsilon_{m}+2(z-1)+2 \eta_{\varphi_{N}}\right],
$$

in $d=3$. Therefore, to obtain the explicit form of this $\beta$ function in terms of the disorder couplings, we have to compute the DSE and the anomalous dimension of the disorder field. The former is obtained from the fermion selfenergy with the diagram shown in Fig. 9(a), while the latter is found from the vertex diagram in Fig. 9(b). Evaluation of these two diagrams has been carried out using the fieldtheoretic method (see Appendix C). Alternatively, one may choose to integrate out the fast modes within the momentum shell $\Lambda e^{-l}<k<\Lambda$, with $\Lambda$ as an ultraviolet cutoff in the momentum, to arrive at the RG flow equations for $\Delta_{N}$. We note that in the $\epsilon_{m}$ expansion two ladder diagrams shown in Figs. 5(c) and 5(d) are ultraviolet convergent (see Appendix C 3) irrespective of the choice of disorder vertices. Therefore, during the coarse graining no new or short-range disorder gets generated (see also Appendix G 1). This conclusion remains operative even beyond the leading order in $\epsilon_{m}$ expansion.

\section{Self-energy and dynamic scaling exponent}

We first show the computation of the self-energy diagram, shown in Fig. 9(a), yielding the dynamical exponent and the anomalous dimension for the fermion field within the regularization scheme defined by the parameter $\epsilon_{m}=1-m$, the deviation from the critical disorder distribution. All the integrals are therefore performed in $d=3$. The divergent part of the integral appears as a pole $\sim 1 / \epsilon_{m}$, analogously to the case of the dimensional regularization where the deviation from the upper or lower 


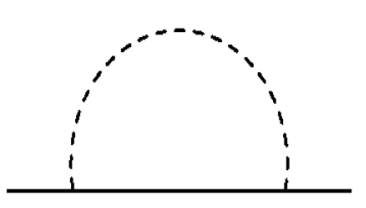

(a)

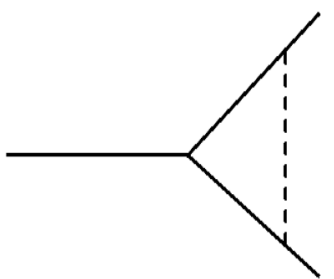

(b)
FIG. 9. One-loop (a) self-energy and (b) vertex diagram. Contributions from only these two diagrams are ultraviolet divergent in $\epsilon_{m}=m-1$ expansion. Evaluations of these two diagrams are shown in Appendix C. Here, solid (dashed) lines represent fermion (disorder) fields.

critical space-time dimension plays the role of an expansion parameter. To find renormalization constants, we use minimal subtraction; i.e., we keep only the divergent part appearing in the corresponding diagrams.

The action [see Eq. (16)] without the disorder yields the inverse free-fermion propagator $G_{0}^{-1}(i \omega, \mathbf{k})=i\left(\gamma_{0} \omega+\right.$ $v_{0} \gamma_{j} k_{j}$ ), with $v_{0}$ as the bare Fermi velocity. Taking into account the self-energy correction, the inverse dressed fermion propagator is

$$
G^{-1}(i \omega, \mathbf{k})=G_{0}^{-1}(i \omega, \mathbf{k})+\Sigma(i \omega, \mathbf{k}),
$$

with $\Sigma(i \omega, \mathbf{k})$ as the self-energy. After accounting for all possible disorders, we arrive at the following compact expression for the self-energy (see Appendix C for details):

$$
\Sigma(i \omega, \boldsymbol{k})=i \gamma_{0} \omega\left(\frac{f_{1}\left(\Delta_{j}\right)}{\epsilon_{m}}\right)+i v_{0} \gamma_{j} k_{j}\left(\frac{f_{2}\left(\Delta_{j}\right)}{3 \epsilon_{m}}\right),
$$

where

$$
\begin{aligned}
f_{1}\left(\Delta_{j}\right)= & \Delta_{V}+\Delta_{A}+3 \Delta_{M}+3 \Delta_{C}+3 \Delta_{S O}+3 \Delta_{A M} \\
& +\Delta_{S}+\Delta_{P S}, \\
f_{2}\left(\Delta_{j}\right)= & -\Delta_{V}-\Delta_{A}+\Delta_{M}+\Delta_{C}-\Delta_{S O}-\Delta_{A M} \\
& +\Delta_{S}+\Delta_{P S},
\end{aligned}
$$

with $\hat{\Delta}_{j}=\Delta_{j} k^{\epsilon_{m}} /\left(2 \pi^{2} v^{2}\right)$ as the dimensionless disorder strength, and for brevity we here drop the hat symbol in the final expression. From the above expression of the selfenergy, together with the renormalization condition $G^{-1}(\omega, \mathbf{k})=Z_{\Psi}\left(i \gamma_{0} \omega+Z_{v} v i \gamma_{j} k_{j}\right)$, with $v$ as the renormalized Fermi velocity, we arrive at the expression for the fermion-field renormalization $\left(Z_{\Psi}\right)$ and velocity renormalization $\left(Z_{v}\right)$ :

$Z_{\Psi}=1+\frac{f_{1}\left(\Delta_{j}\right)}{\epsilon_{m}}, \quad Z_{v}=1-\frac{1}{\epsilon_{m}}\left(f_{1}\left(\Delta_{j}\right)-\frac{f_{2}\left(\Delta_{j}\right)}{3}\right)$
This equation then yields the anomalous dimension for the fermion field:

$$
\eta_{\Psi}=-\sum_{j} \frac{d \ln Z_{\Psi}}{d \Delta_{j}} \beta_{\Delta_{j}}
$$

Furthermore, the renormalization factor $Z_{v}$ enters the renormalization condition for the Fermi velocity $Z_{v} v=v_{0}$. Using Eq. (26), together with $\beta_{\Delta_{N}}=-\epsilon_{m} \Delta_{N}+$ $\mathcal{O}\left(\Delta_{j}^{2}\right)$, we find

$$
\beta_{v}=-\frac{1}{3} v\left[3 f_{1}\left(\Delta_{j}\right)-f_{2}\left(\Delta_{j}\right)\right] .
$$

Finally, the $\beta$ function of the Fermi velocity is $\beta_{v}=(1-z) v$, which together with Eq. (28) determines the DSE:

$$
z=1+\frac{1}{3}\left[3 f_{1}\left(\Delta_{j}\right)-f_{2}\left(\Delta_{j}\right)\right]
$$

\section{Vertex correction: Anomalous dimension of disorder field}

We now turn to the vertex correction due to the disorder, shown in Fig. 9(b), which yields the anomalous dimension of the disorder field. As shown in Appendix C, the vertex represented by the matrix $N$ receives the correction of the form

$$
V_{N}(\mathbf{k})=\sum_{M}\left[M \gamma_{j} N \gamma_{j} M\right] \frac{\Delta_{M}}{3 \epsilon_{m}}
$$

The corresponding renormalization condition that determines the renormalization constant $Z_{\varphi_{N}}$ for the disorder field reads

$$
Z_{\Psi} Z_{\varphi_{N}} N+V_{N}=N
$$

with $Z_{\Psi}$ given by Eq. (26). The above condition in turn yields the anomalous dimension of the disorder field as

$$
\eta_{\varphi_{N}}=-\sum_{j} \frac{d \ln Z_{\varphi_{N}}}{d \Delta_{j}} \beta_{\Delta_{j}}
$$

which we then use to write the explicit form of the $\beta$ function, given by Eq. (21) in terms of the disorder couplings.

\section{C. $\epsilon_{d}$ expansion about $d=2$}

Alternatively, one may take the Gaussian white noise distribution in Eq. (17) with $m \rightarrow 0$ from the outset. In that case, the engineering dimension of the disorder coupling is equal to $2-d$, since $z=1$ in a clean WSM. Therefore, $d=2$ is the lower critical dimension in the problem and we can use $\epsilon_{d}=d-2$ as an expansion parameter, following 
the spirit of $\epsilon$ expansion [30,36,37,40,42,45,56,79,84]. In this scheme, after performing the disorder averaging using the replica method, the imaginary time action assumes a similar form of Eq (10).

Within the framework of the $\epsilon_{d}$ expansion only the temporal (frequency-dependent) component of self-energy acquires a disorder-dependent correction to the leading order. The self-energy correction due to disorder reads as

$$
\Sigma(i \omega, \boldsymbol{k})=i \gamma_{0} \omega\left(\frac{f_{1}\left(\Delta_{j}\right)}{\epsilon_{d}}\right),
$$

with the function $f_{1}\left(\Delta_{j}\right)$ given by Eq. (24), and $\Delta_{j} \Lambda^{\epsilon_{d}} /\left(2 \pi v^{2}\right) \rightarrow \Delta_{j}$. This result is obtained from Eq. (C1) with $d=2+\epsilon_{d}$ and $m=0$. As a result, the field-renormalization factor $Z_{\Psi}=1+f_{1}\left(\Delta_{j}\right) / \epsilon_{d}$ and the velocity renormalization factor is $Z_{v}=1-f_{1}\left(\Delta_{j}\right) / \epsilon_{d}$. Using the renormalization condition $Z_{v} v=v_{0}$, together with $\beta_{\Delta_{N}}=-\epsilon_{d} \Delta_{N}+\mathcal{O}\left(\Delta_{j}^{2}\right)$, we obtain the leading-order RG flow equation for the Fermi velocity,

$$
\beta_{v}=v(1-z)=-v f_{1}\left(\Delta_{j}\right),
$$

which yields a scale-dependent dynamic exponent $z=1+f_{1}\left(\Delta_{j}\right)$. The seemingly different expressions for the flow equation and DSE in these two schemes stems from underlying different methodology of capturing the ultraviolet divergences of various diagrams. However, such details do not alter any physical outcome. While extracting the RG flow of all disorder couplings, we first complete the $\gamma$ matrix algebra in $d=3$ and subsequently perform the momentum integral in $d=2+\epsilon$. Such a procedure is safe at least to the leading order in $\epsilon_{d}$ expansion as the relevant Feynman diagrams (see Fig. 5) do not contain any overlapping divergence. For next-to-leading-order calculation one also needs to perform the $\gamma$-matrix algebra in $d=2+\epsilon$. However, in the $\epsilon_{m}$-expansion scheme we do not need to continue the $\gamma$ matrix algebra in general dimension, as the entire analysis is performed in $d=3$.

\section{CHIRAL-SYMMETRIC OR INTRANODE DISORDER}

We first focus on chiral-symmetric disorders. For a single pair of Weyl fermions there are four such disorders, namely, chemical potential, axial potential, current, and axial current disorders, as shown in Table III. With appropriate lattice model axial current disorder corresponds to magnetic impurities, and from here onward we use this terminology. We address the effect of weak and strong chiral-symmetric disorder using both $\epsilon_{m}$ and $\epsilon_{d}$ expansions.

\section{A. $\epsilon_{m}$ expansion}

Let us first analyze this problem pursuing the $\epsilon_{m}$ expansion. Using Eqs. (21), (29), (31), and (32), we obtain

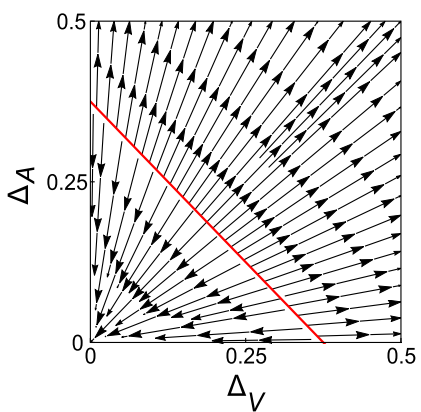

(a)

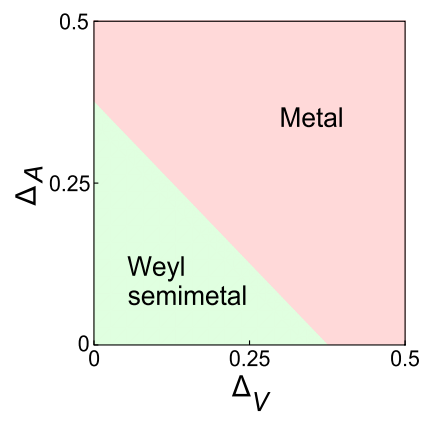

(b)
FIG. 10. (a) The RG flow diagram and (b) the phase diagram in the $\Delta_{V}-\Delta_{A}$ plane, for $\epsilon_{m}=1$, obtained from Eq. (35). Here $\Delta_{V}$ and $\Delta_{A}$ are, respectively, the strength of potential and axial potential disorder. The red line in (a) corresponds to the line of quantum-critical points [see Eq. (36)] that in turn defines the phase boundary between the Weyl semimetal and metallic phases, as shown in (b). A similar flow and phase diagram is obtained from the RG calculation performed within the framework of an $\epsilon_{d}$ expansion [see Eq. (39)] [30,42,44].

the following RG flow equations for the coupling constants to the leading order in $\epsilon_{m}$ :

$\beta_{\Delta_{V}}=\Delta_{V}\left(-\epsilon_{m}+\frac{8}{3}\left(\Delta_{V}+\Delta_{A}\right)+\frac{16}{3}\left(\Delta_{C}+\Delta_{M}\right)\right)$,

$\beta_{\Delta_{A}}=\Delta_{A}\left(-\epsilon_{m}+\frac{8}{3}\left(\Delta_{V}+\Delta_{A}\right)+\frac{16}{3}\left(\Delta_{C}+\Delta_{M}\right)\right)$,

$\beta_{\Delta_{M}}=-\epsilon_{m} \Delta_{M}, \quad \beta_{\Delta_{C}}=-\epsilon_{m} \Delta_{C}$.

The above set of flow equations supports a line of quantumcritical points in the $\Delta_{V}-\Delta_{A}$ plane, determined by

$$
\Delta_{V, *}+\Delta_{A, *}=\frac{3}{8} \epsilon_{m},
$$

where the quantities with subscript “*” represent their critical values for WSM-metal QPT. The RG flow in this plane is shown in Fig. 10(a). The line of QCPs also determines the WSM-metal phase boundary, and the corresponding phase diagram in the $\Delta_{V}-\Delta_{A}$ plane is shown in Fig. 10(b). At each point of this line of QCPs the DSE and CLE are respectively given by

$$
z=1+\frac{\epsilon_{m}}{2}+\mathcal{O}\left(\epsilon_{m}^{2}\right), \quad \nu^{-1}=\epsilon_{m}+\mathcal{O}\left(\epsilon_{m}^{2}\right) .
$$

Therefore, for the Gaussian white noise distribution, realized for $\epsilon_{m}=1$, we obtain $z=3 / 2$ and $\nu=1$ from the leading-order $\epsilon_{m}$ expansion. If the bare value of either the chemical potential or axial potential disorder strength is zero, the quantum-critical behavior is governed by the QCP corresponding to the disorder of a nonvanishing bare value [44]. This QCP features the critical exponents of the same 


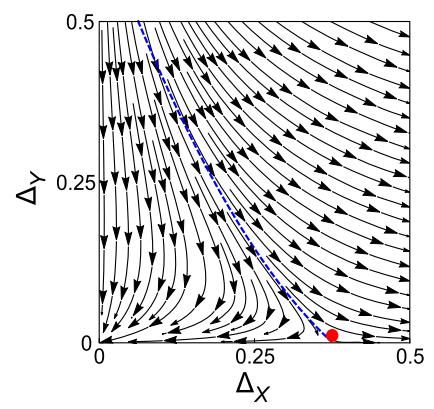

(a)

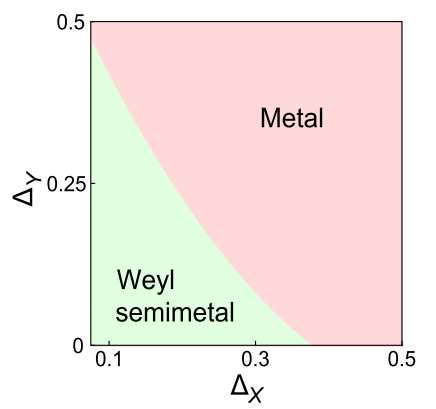

(b)
FIG. 11. (a) The renormalization-group flow diagram and (b) corresponding phase diagram in the $\Delta_{X}-\Delta_{Y}$ plane, where $X=V, A$ and $Y=M, C$ obtained from Eq. (35). In these planes there is only one QCP at $\Delta_{X}=3 \epsilon_{m} / 8, \Delta_{Y}=0$ (red dot). The phase boundary between the Weyl semimetal and metal in (b) is determined by the irrelevant direction, shown by blue dotted line in (a).

value to the one-loop order, as in the case of the quantumcritical line, given by Eq. (37).

From the RG flow equations [see Eq. (35)], we find that both magnetic and current disorder are always irrelevant perturbations, at least to the leading order in the $\epsilon_{m}$ expansion. In the $\Delta_{X}-\Delta_{Y}$ plane, where $X=V, A$ and $Y=M, C$, the RG flow diagram is shown in Fig. 11(a) and the corresponding phase diagram is shown in Fig. 11(b). Importantly, the QPT separating the metallic and the semimetallic phase in any $\Delta_{X}-\Delta_{Y}$ plane is governed by the QCP located at $\Delta_{X, *}=3 \epsilon_{m} / 8$. The phase boundary between these two phases is determined by the irrelevant direction at this QCP. Therefore, across the entire WSM-metal phase boundary in these planes the universality class of the QPT is identical and characterized by $z=1+\epsilon_{m} / 2+\mathcal{O}\left(\epsilon_{m}^{2}\right)$ and $\nu^{-1}=\epsilon_{m}+\mathcal{O}\left(\epsilon_{m}^{2}\right)$ to the leading order in $\epsilon_{m}$ expansion.

\section{B. $\epsilon_{d}$ expansion}

The RG flow equations for the chiral-symmetric disorder coupling constants within the framework of the leadingorder $\epsilon_{d}$ expansion are

$$
\begin{aligned}
& \beta_{\Delta_{V}}=\Delta_{V}\left[-\epsilon_{d}+2 F_{+}\left(\Delta_{j}\right)\right]+8 \Delta_{M} \Delta_{C}, \\
& \beta_{\Delta_{A}}=\Delta_{A}\left[-\epsilon_{d}+2 F_{+}\left(\Delta_{j}\right)\right]+4\left(\Delta_{M}^{2}+\Delta_{C}^{2}\right), \\
& \beta_{\Delta_{M}}=\Delta_{M}\left[-\epsilon_{d}+\frac{2}{3} F_{-}\left(\Delta_{j}\right)\right]+\frac{8}{3}\left(\Delta_{C} \Delta_{V}+\Delta_{A} \Delta_{M}\right), \\
& \beta_{\Delta_{C}}=\Delta_{C}\left[-\epsilon_{d}+\frac{2}{3} F_{-}\left(\Delta_{j}\right)\right]+\frac{8}{3}\left(\Delta_{C} \Delta_{V}+\Delta_{A} \Delta_{M}\right),
\end{aligned}
$$

where $F_{ \pm}\left(\Delta_{j}\right)=\left(\Delta_{V}+\Delta_{A}\right) \pm\left(\Delta_{C}+\Delta_{M}\right)$. These coupled flow equations also support only a line of QCPs in the $\Delta_{V}-\Delta_{A}$ plane, as we previously found from Eq. (35) using $\epsilon_{m}$ expansion, now determined by

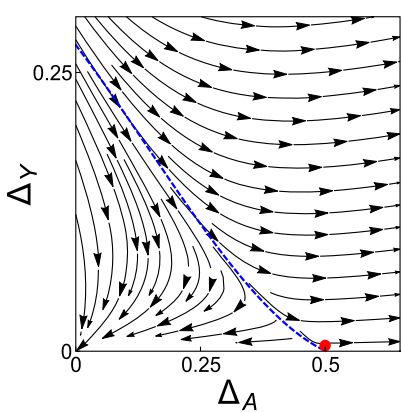

(a)

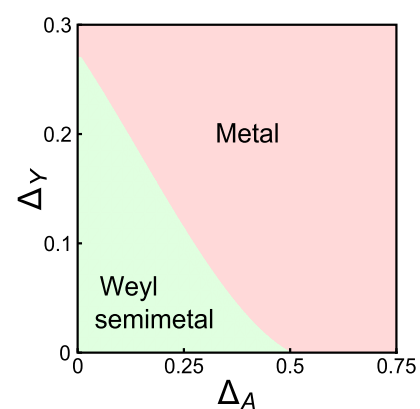

(b)
FIG. 12. (a) The renormalization-group flow diagram and (b) corresponding phase diagram in the $\Delta_{A}-\Delta_{Y}$ plane, where $Y=M, C$ obtained from $\epsilon_{d}$ expansion for $\epsilon_{d}=1$. There is only one quantum-critical point at $\Delta_{A}=\epsilon_{d} / 2, \Delta_{Y}=0$ (red dot). The phase boundary between the Weyl semimetal and metal in (b) is determined by the irrelevant direction, shown by the blue dotted line in (a). These figures are qualitatively similar to the ones shown in Fig. 11, apart from the nonuniversal shift in the phase boundary.

$$
\Delta_{V, *}+\Delta_{A, *}=\frac{\epsilon_{d}}{2},
$$

similar to the one in Eq. (36). The critical exponents at each point of such line of QCPs are $z=1+\epsilon_{d} / 2+\mathcal{O}\left(\epsilon_{d}^{2}\right)$ and $\nu^{-1}=\epsilon_{d}+\mathcal{O}\left(\epsilon_{d}^{2}\right)$. We here stress that presently there is no known method to compute these two exponents beyond leading order in $\epsilon_{d}$ in a controlled fashion [40,45]. Therefore, in three spatial dimensions, $\epsilon_{d}=1$, and we find $z=3 / 2$ and $\nu=1[30,42]$. The RG flow diagram and the corresponding phase diagram are similar to the ones shown in Figs. 10(a) and 10(b). Only the location of the line of QCPs and the phase boundary shift in a nonuniversal fashion. The differences in the flow equations [Eqs. (36) and (38)] arise from two diagrams shown in Figs. 5(c) and 5(d), which produce ultraviolet divergent contributions, but only within the $\epsilon_{d}$-expansion scheme. In the presence of only potential disorder, we find $z=3 / 2$ and $\nu=1$ $[30,36,37,40,42,45]$.

Notice that if we start with only magnetic or current disorder, the axial disorder gets generated from Feynman diagrams in Figs. 5(c) and 5(d). Thus, to close the RG flow equations, we need to account for $\Delta_{A}$ coupling from the outset, and the resulting RG flow equations read

$$
\begin{aligned}
& \beta_{\Delta_{A}}=\Delta_{A}\left[-\epsilon_{d}+2\left(\Delta_{A}+3 \Delta_{Y}\right)\right]+4 \Delta_{Y}^{2}, \\
& \beta_{\Delta_{Y}}=\Delta_{Y}\left[-\epsilon_{d}+\frac{2}{3}\left(\Delta_{Y}-\Delta_{A}\right)\right]+\frac{8}{3} \Delta_{A} \Delta_{Y},
\end{aligned}
$$

for $Y=M, C$. The above set of coupled RG flow equations supports only one QCP, located at $\Delta_{A, *}=\epsilon_{d} / 2, \Delta_{Y}=0$. The RG flow and the resulting phase diagrams are shown in Figs. 12(a) and 12(b), respectively. Hence, in the presence of magnetic and current disorder, the transition to the 
metallic phase is controlled by the QCP due to axial disorder. If we also take into account the presence of potential disorder, then such a semimetal-metal QPT takes place through one of the points residing on the line of QCPs in the $\Delta_{V}-\Delta_{A}$ plane, depending on the bare relative strength of these two disorder couplings.

\section{Chiral superuniversality}

From the discussion in the previous two sections, we can conclude that in the presence of chiral-symmetric disorder in a WSM, the semimetal-metal QPT takes place through either a QCP or a line of QCPs. The location of the line of QCPs and the resulting phase boundaries are nonuniversal and thus dependent on the RG scheme. However, the universal quantum-critical behavior with chiral-symmetric disorder couplings is insensitive to these details, at least to the leading order in the expansion parameter, and all QPTs in the fourdimensional hyperplane of disorder coupling constants are characterized by an identical set of critical exponents, namely, $z=1+\epsilon / 2+\mathcal{O}\left(\epsilon^{2}\right)$ and $\nu^{-1}=\epsilon+\mathcal{O}\left(\epsilon^{2}\right)$, with $\epsilon=1$. The importance of the higher-order corrections is presently unknown. Therefore, emergent quantum-critical behavior for strong chiral-symmetric disorder stands as a rare example of superuniversality, and we name it chiral superuniversality. Next, we demonstrate emergence of such superuniversality across WSM-metal QPT by numerically analyzing the scaling of average DOS in the presence of generic chiral-symmetric disorder.

\section{NUMERICAL DEMONSTRATION OF CHIRAL SUPERUNIVERSALITY}

Motivated by the field-theoretic prediction of emergent chiral superuniversality across the WSM-metal QPTs driven by CSP disorder, next we numerically investigate the scaling of average DOS across such QPTs. Since $\varrho(0)$ vanishes and is finite in the WSM and metallic phases, respectively, it can be promoted as a bona fide order parameter across the WSM-metal QPT [52,54,56,57,59,63]. In addition, such analysis endows an opportunity to extract the critical exponents for the transition nonperturbatively and, at the same time, test the validity of the proposed scenario for chiral superuniversality. The WSM phase is realized from the tight-binding model, defined through Eqs. (3) and (5), which we implement on a cubic lattice of linear dimension $L$. For numerical analysis we always set $m_{z}=0$, and for current disorder take $t=t_{z}=1=t_{0}$, while $t=1=t_{0}$, $t_{z}=\frac{1}{2}$ for the remaining seven types of elastic scatterers [see Table III], in the clean model, given by Eqs. (2)-(5). We use lattice realizations of disorder introduced in Appendix D. We impose periodic boundary condition in all three directions. The average DOS is computed by using the kernel polynomial method [72]. The average is taken over 20 random realizations of disorder that minimizes the residual statistical error in average DOS, which is a self-averaged quantity. We typically compute 4096 Chebyshev moments and take the trace over $\sim 12$ random vectors to compute the average DOS. All types of disorder are distributed uniformly and randomly within the range $[-W, W]$. The scaling theory for average DOS has already been discussed in Sec. III B. Thus, we can readily start from the final expression of the general scaling form of the average DOS, presented in Eq. (14), and continue with our numerical analysis.

\section{A. Numerical analysis with random intra-node scatterers or chiral-symmetric disorder}

We begin the discussion on the effects of randomness on WSM by focusing on the intranode or chiral-symmetric disorder. Let us first focus on the quantum-critical regime and for now we assume that the system size is sufficiently large so that we can neglect the $L$ dependence in Eq. (14). In this regime the scaling function must be independent of $\delta$, dictating $F(x) \sim x^{d / z-1}$. Therefore, when $W=W_{c}$ we compare $\varrho(E)$ versus $E^{d / z-1}$ and extract the DSE $z$. Such analysis for all four possible CSP disorders is shown in the first column of Fig. 13 and numerically extracted values of $z$ are quoted in Table I. Within the numerical accuracy, we always find $z \approx 1.5$, in excellent agreement with the field-theoretic result, obtained from the leading-order $\epsilon$ expansions.

Next we proceed to the metallic side of the transition, where average DOS at zero energy becomes finite. From the scaling function in Eq. (14), we obtain $\varrho(0) \sim \delta^{(d-z) \nu}$. Thus, by comparing $Q(0)$ versus $\delta$, we extract the CLE $\nu$, using already-obtained value of the $\operatorname{DSE} z$, as shown in the second column of Fig. 13. The numerically found CLE is also quoted in Table I, and within numerical accuracy $\nu \approx 1$ always, irrespective of the nature of CSP disorder. Once again we find an excellent agreement of numerically extracted values of $\nu$ with the one obtained from the leading-order $\epsilon$ expansions. These two results strongly support the picture of chiral superuniversality.

To test the quality of our numerical analysis we search for two types of data collapse. First, we compare $\varrho(E)|\delta|^{-\nu(d-z)}$ versus $|\delta|^{-\nu z}|E|$, motivated by the scaling form of average DOS, displayed in Eq. (14). Using numerically obtained values of $\nu$ and $z$, we find that for energies much smaller than the bandwidth $(|E| \ll 1)$, all data collapse onto two separate branches for all four disorders, as shown in the third column of Fig. 13. While the top branch corresponds to the metallic phase, the lower one stems from the WSM phase, and eventually these two branches meet in the quantum-critical regime.

Finally, we demonstrate a finite-size data collapse for $Q(0)$ for different system sizes $(L)$ by focusing on the metallic side of the transition. Setting $E=0$ in Eq. (14), we obtain $\varrho(0)=L^{z-d} F\left(0, \delta L^{1 / \nu}\right)$. Hence, we compare $\varrho(0) L^{d-z}$ versus $\delta L^{1 / \nu}$ and find an excellent data collapse 

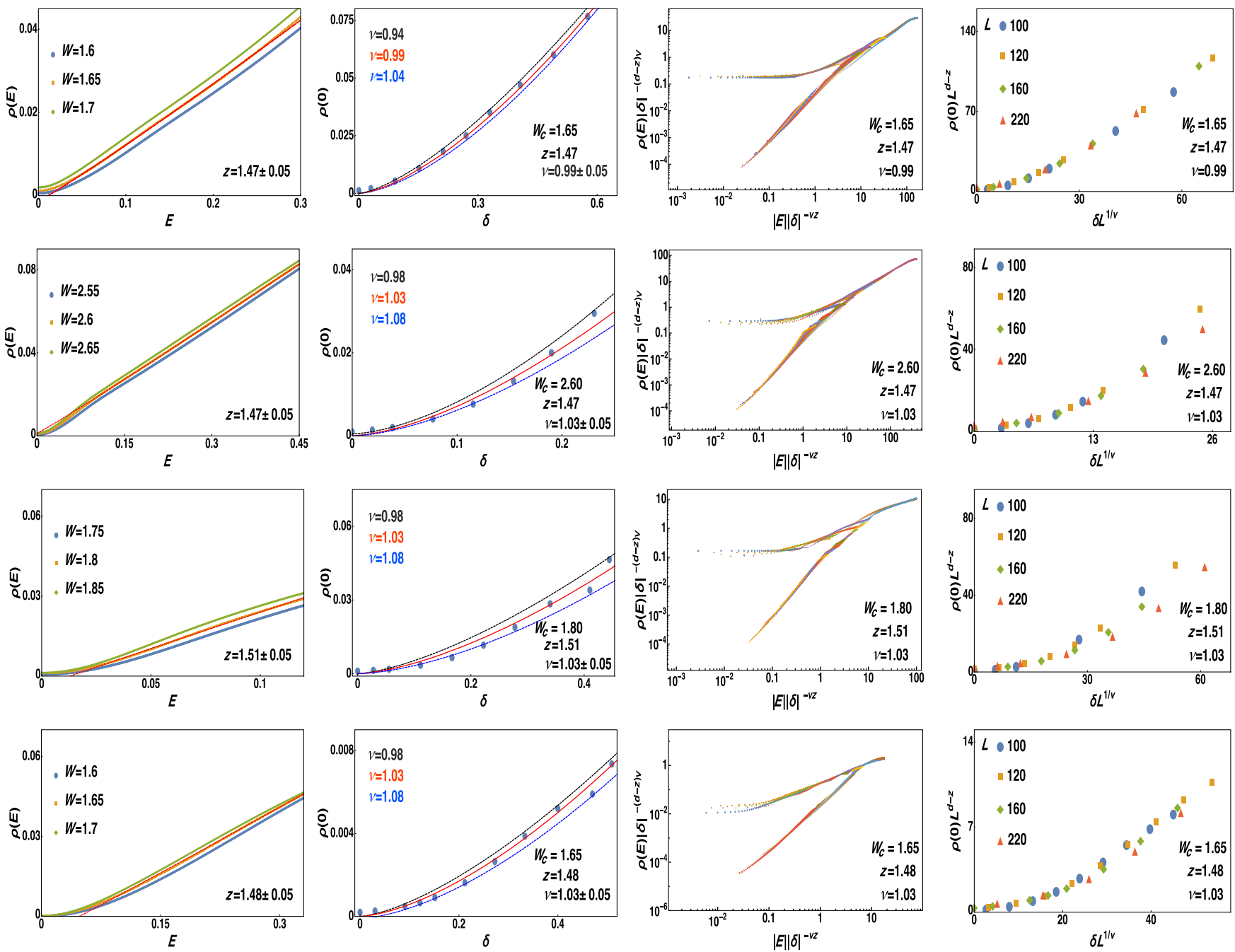

FIG. 13. Scaling analysis of average density of states in various regimes of the phase diagram of a dirty WSM for all four possible intranode scatterings; plots from top to bottom rows correspond to potential $\left(\Delta_{V}\right)$, axial potential $\left(\Delta_{A}\right)$, axial current $\left(\Delta_{M}\right)$, and current $\left(\Delta_{C}\right)$ disorder. First column shows the scaling of ADOS $\varrho(E)$ versus $E$ around the critical strength of disorder $\left(W=W_{c}\right)$. The second column depicts the scaling of ADOS at zero energy $\varrho(0)$ versus $\delta$, the reduced distance from the critical disorder defined as $\delta=\left[\left(W-W_{c}\right) / W_{c}\right]$. In the third column we display $\varrho(E) \delta^{-(d-z) \nu}$ versus $|E||\delta|^{-\nu z}$ for weak $\left(W<W_{c}\right)$ and strong $\left(W>W_{c}\right)$ disorder and $|E| \ll t(=1)$. All data collapse onto two branches. The top branch represents the metallic phase, while the lower branch represents WSM. Note that these two branches meet at large values of $|E||\delta|^{-\nu z}$, corresponding to the quantum-critical regime. All data in the first three columns are obtained from a system of linear dimension $L=220$. The finite-size data collapse inside the metallic phase is shown in the fourth column, where we compare $\varrho(0) L^{d-z}$ versus $\delta L^{1 / \nu}$ for $100 \leq L \leq 220$. Notice that all data collapse onto one branch for small to moderate values of $\delta L^{1 / \nu}$, with the numerically extracted values of the critical exponents $z$ and $\nu$, quoted in the figure and summarized in Table I. The quality of the data collapse progressively worsens for larger values of $\delta L^{1 / \nu}$ due to the existence of a second QPT of a three-dimensional dirty Weyl metal into the Anderson insulating phase, discussed in Sec. IX. Scaling of ADOS and data analysis are discussed in detail in Sec. VI. The quoted error bars in $z$ and $\nu$ are fitting error bars. See Appendix E and Table IV (first four rows) for details of data analysis.

for $100<L<220$, using numerically obtained values of $\nu$ and $z$ for all four disorders, as shown in the fourth column of Fig. 13. The data collapse becomes systematically worse for large values of $\delta$ or stronger disorder due to the existence of a second transition that takes the system from a metallic phase to an Anderson insulator. Therefore, our thorough numerical analysis provides a valuable and unprecedented insight into the nature of the WSM-metal
QPTs driven by generic chiral-symmetric disorder, and staunchly supports the proposal of an emergent chiral superuniversality across such QPTs.

Finally, we note that one can attempt to extract the CLE $(\nu)$ from the scaling of ADOS at finite energy in the semimetallic side of the transition in the following way. In the WSM phase the universal scaling function (after neglecting the $L$ dependence) $F(x) \sim x^{d-1}$, yielding 

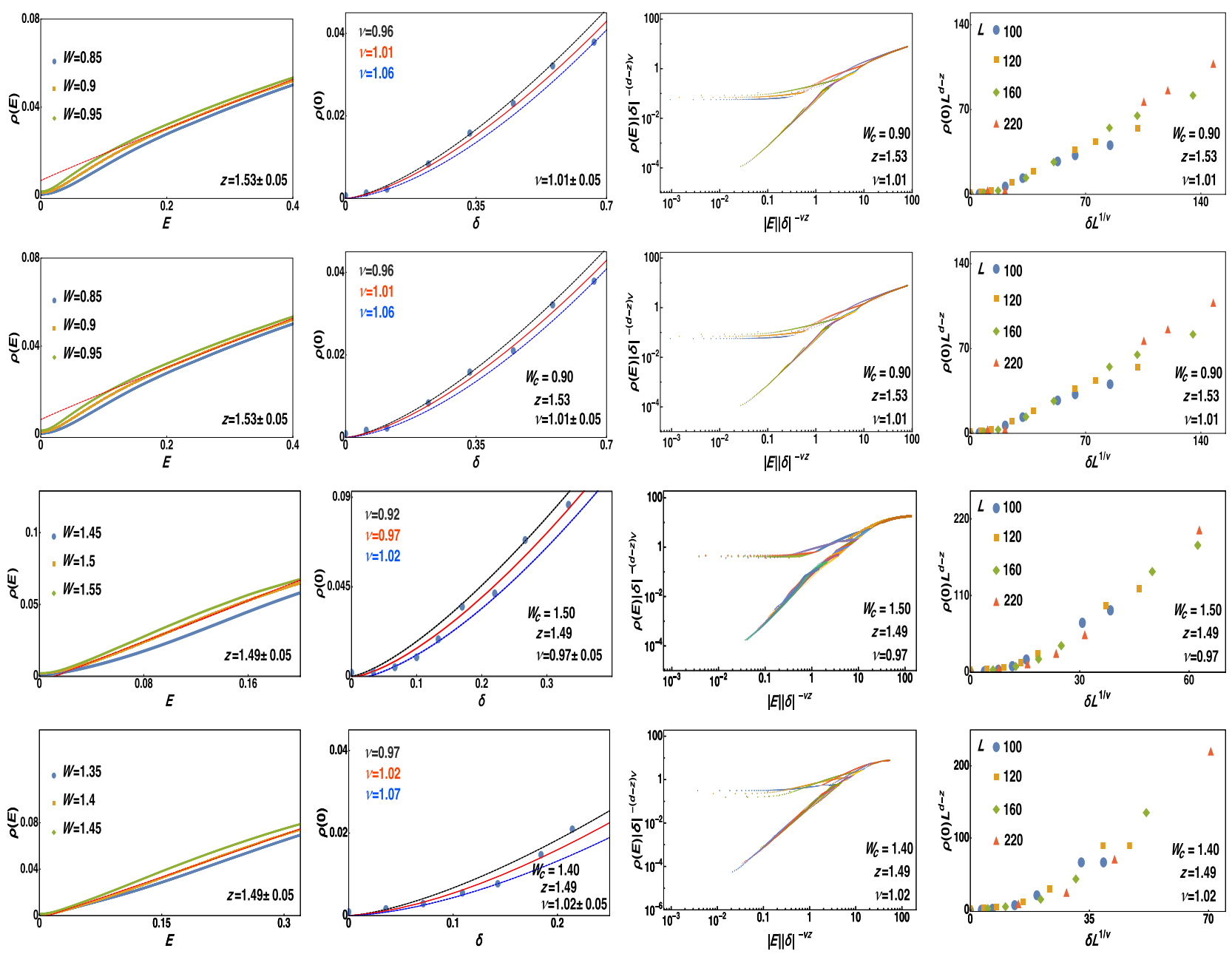

FIG. 14. Scaling analysis of numerically extracted ADOS in various regimes of the phase diagram of a dirty WSM in the presence of internode scattering. Each column is identical to the corresponding one in Fig. 13 (including methods of analysis and system size). The plots from top to bottom rows correspond to temporal $\left(\Delta_{S O}\right)$ and spatial $\left(\Delta_{A M}\right)$ component of tensor, scalar $\left(\Delta_{S}\right)$, and pseudoscalar $\left(\Delta_{P S}\right)$ mass disorder (see Table III). Final results of our analysis are quoted in Table II. The quoted error bars in $z$ and $\nu$ are fitting error bars. See also Appendix E and Table IV (from fourth to eighth row) for additional details.

$\varrho(E) \sim \delta^{(1-z) d \nu}|E|^{d-1}$; see Eq. (14) for sufficiently small energy. By contrast, for moderately high energy (still $|E| \ll 1), \varrho(E) \sim|E|$ inside the critical regime. Therefore, by tracking the scaling of the crossover boundary between the WSM [displaying $\varrho(E) \sim|E|^{2}$ ] and critical regime [displaying $Q(E) \sim|E|^{2}$ ] at finite energy for subcritical disorder, one can extract the CLE $\nu$. However, determination of such crossover boundary does not rest on any strict criterion and is often (if not always) associated with a large error, which in turn produces a large error bar in the determination of CLE $[52,56,57,59]$. Therefore, this methodology of determining $\nu$ and the corresponding error bar is questionable.

\section{B. Numerical analysis with random internode scatterers or nonchiral disorder}

Motivated by the intriguing possibility of realizing an emergent superuniversality, we further seek to examine its robustness in the presence of internode scattering (also referred to as nonchiral disorder). In the simplest version of a Weyl semimetal composed of only two Weyl nodes, there are four sources of internode scattering, highlighted in Table III, and their lattice realization is shown in Appendix D. We rely on the scaling of average DOS in the presence of nonchiral disorder as well, and all the parameters and numerical strategies are identical to the ones pursued for chiral-symmetric (intranode) disorder. The analyses of average DOS in various regimes of the phase diagram of disordered WSM are performed in the same fashion. The locations of WSM-metal QPT are shown in Fig. 22 (lower row), and numerically extracted values of two critical exponents $\nu$ and $z$ are reported in Table II. The details of the data analysis are displayed in Fig. 14.

Within the numerical accuracy we find that the WSMmetal QPT driven by CSB disorder is also characterized by $\nu \approx 1$ and $z \approx 1.5$. Therefore, the chiral superuniversality 
appears to be generic in a dirty WSM, and the WSM-metal QPTs belong to the same universality class, irrespective of the nature of impurities. Such an intriguing outcome further motivates us to understand the effect of internode scattering in a WSM from a field-theoretic point of view, which we present in the following section by carrying out two different $\epsilon$ expansions, described in Secs. IV B and IV C.

\section{CHIRAL-SYMMETRY-BREAKING OR INTERNODE DISORDER}

In a WSM constituted by a single pair of Weyl nodes, there are four CSB disorders, namely temporal and spatial components of a tensor disorder, which in a suitable lattice model respectively represent spin-orbit and axial magnetic disorder, as well as scalar and pseudoscalar mass disorder; see Table III. We address the effects of weak and strong CSB disorder by using both $\epsilon_{m}$ and $\epsilon_{d}$ expansions.

\section{A. $\epsilon_{m}$ expansion}

Within the framework of an $\epsilon_{m}$ expansion, the RG flow equations to one-loop order read as

$$
\begin{aligned}
\beta_{\Delta_{S O}} & =\Delta_{S O}\left[-\epsilon_{m}+\frac{4}{3}\left(\Delta_{A M}-\Delta_{S}\right)\right], \\
\beta_{\Delta_{A M}} & =\Delta_{A M}\left[-\epsilon_{m}+\frac{4}{3}\left(\Delta_{S O}-\Delta_{P}\right)\right], \\
\beta_{\Delta_{S}} & =\Delta_{S}\left[-\epsilon_{m}+\frac{4}{3}\left(5 \Delta_{S O}-4 \Delta_{A M}-2 \Delta_{S}+\Delta_{P S}\right)\right], \\
\beta_{\Delta_{P S}} & =\Delta_{P S}\left[-\epsilon_{m}+\frac{4}{3}\left(5 \Delta_{A M}-4 \Delta_{S O}-2 \Delta_{P S}+\Delta_{S}\right)\right] .
\end{aligned}
$$

Therefore, individually each CSB disorder is always an irrelevant perturbation, at least to the leading order in the $\epsilon_{m}$ expansion, and as such does not lead to any QPTs. However, in the absence of chiral symmetry all four disorder couplings are present, and to address the critical properties in this situation, we recast the above flow equations in terms of newly defined coupling constants as

$$
\begin{aligned}
& \beta_{\Delta_{V}^{+}}=-\epsilon_{m} \Delta_{V}^{+}+\frac{2}{3}\left[\left(g_{V}^{+}\right)^{2}-\left(\Delta_{V}^{-}\right)^{2}-\Delta_{V}^{+} \Delta_{S}^{+}-\Delta_{V}^{-} \Delta_{S}^{-}\right] \\
& \beta_{\Delta_{V}^{-}}=-\epsilon_{m} \Delta_{V}^{-}-\frac{2}{3}\left[\Delta_{V}^{-} \Delta_{S}^{+}+\Delta_{V}^{+} \Delta_{S}^{-}\right] \\
& \beta_{\Delta_{S}^{+}}=-\epsilon_{m} \Delta_{S}^{+}-\frac{2}{3}\left[\left(\Delta_{S}^{+}\right)^{2}+3\left(\Delta_{S}^{-}\right)^{2}-\Delta_{V}^{+} \Delta_{S}^{+}-9 \Delta_{V}^{-} \Delta_{S}^{-}\right] \\
& \beta_{\Delta_{S}^{-}}=-\epsilon_{m} \Delta_{S}^{-}-\frac{2}{3}\left[\Delta_{S}^{-} \Delta_{V}^{+}-4 \Delta_{S}^{+} \Delta_{S}^{-}-9 \Delta_{S}^{+} \Delta_{V}^{-}\right]
\end{aligned}
$$

where $\Delta_{V}^{ \pm}=\Delta_{S O} \pm \Delta_{A M}, \Delta_{S}^{ \pm}=\Delta_{S} \pm \Delta_{P S}$. The above set of RG flow equations supports a line of QCPs determined by the equation

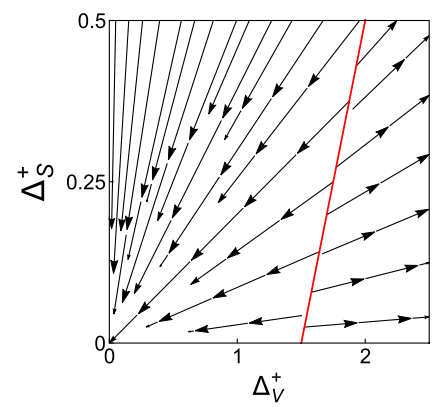

(a)

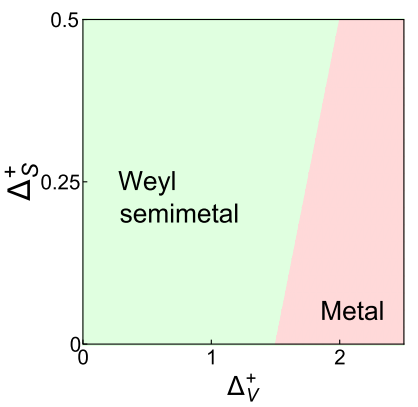

(b)
FIG. 15. (a) The renormalization-group flow diagram and (b) corresponding phase diagram in the $\Delta_{V}^{+}-\Delta_{S}^{+}$plane obtained from $\epsilon_{m}$ expansion for $\epsilon_{m}=1$. The WSM-metal QPT in this coupling constant space is controlled by the line of QCPs [see Eq. (43)], shown by the red line in (a) that in turn also determines the phase boundary between these two phases, as shown in (b).

$\Delta_{V, *}^{+}=\Delta_{S, *}^{+}+\frac{3 \epsilon_{m}}{2}, \quad \Delta_{V, *}^{-}=0, \quad \Delta_{S, *}^{-}=0$.

Notice that if we tune the CSB disorders, so that $\Delta_{V}^{-}=\Delta_{S}^{-}=0$, these two coupling constants do not get generated through quantum corrections, and the plane with $\Delta_{V}^{-}=\Delta_{S}^{-}=0$, shown in Fig. 15, remains invariant under the RG. The RG flow in this plane is shown in Fig. 15(a), and the corresponding phase diagram is presented in Fig. 15(b). The WSM-metal phase boundary in the $\Delta_{V}^{+}-\Delta_{S}^{+}$plane is determined by the line of QCPs, given by Eq. (43), qualitatively similar to the situation in the presence of potential and axial disorders, as shown in Fig. 10. However, these two scenarios are fundamentally different in the sense that while the DSE $z=1+\epsilon / 2$, with $\epsilon=\epsilon_{m}$ or $\epsilon_{d}$, is fixed along the entire line of QCPs in the $\Delta_{V}-\Delta_{A}$ plane, it varies continuously along the line of QCPs in the $\Delta_{V}^{+}-\Delta_{S}^{-}$plane according to

$$
z=1+\frac{2}{3}\left[5 \Delta_{V, *}^{+}+\Delta_{S, *}^{+}\right]=1+5 \epsilon_{m}+4 \Delta_{S, *}^{+},
$$

where the quantity with subscript "** denotes the critical value for WSM-metal transition. Such continuously varying DSE leaves its signature in critical scaling of various physical observables, as we discuss below, and qualitatively mimics the picture of Kosterlitz-Thouless transition. Notice that the end point of such a line of QCPs on the $\Delta_{V}^{+}$ axis resides in the $\Delta_{S O}-\Delta_{A M}$ plane at $\Delta_{S O}=\Delta_{A M}=$ $3 \epsilon_{m} / 4$, and the RG flow in this plane is shown in Fig. 16(a). The phase diagram of a dirty WSM containing only spinorbit and axial magnetic disorder in this plane is shown in Fig. 16(b), with $z=1+5 \epsilon_{m}$, which is directly obtained from Eq. (44) by setting $\Delta_{S}^{+}=0$. It is worth pointing out that in the $\Delta_{S O}-\Delta_{A M}$ plane the phase boundary between the WSM and metallic phase is set by the irrelevant parameter associated with the QCP, while when such 


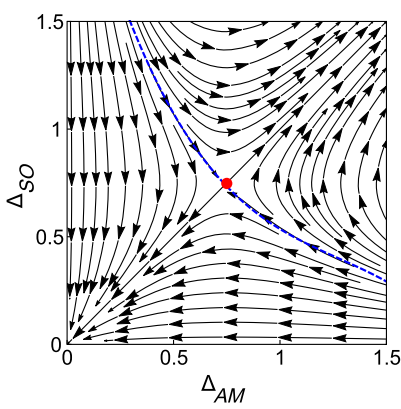

(a)

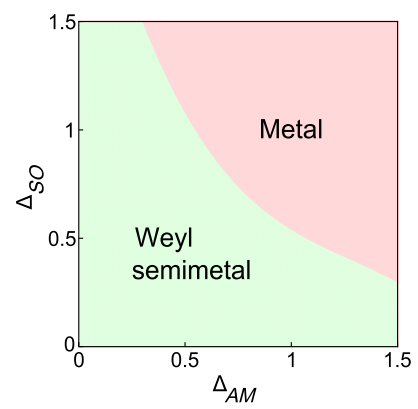

(b)

FIG. 16. (a) The renormalization-group flow diagram and (b) corresponding phase diagram in the $\Delta_{A M}-\Delta_{S O}$ plane obtained from $\epsilon_{m}$ expansion for $\epsilon_{m}=1$. There is only one quantum-critical point at $\Delta_{A M}=\Delta_{S O}=3 \epsilon_{m} / 4$ (red dot). The phase boundary between the Weyl semimetal and metal in (b) is determined by the irrelevant direction, shown by blue dotted line in (a).

QCP percolates through the $\Delta_{V}^{+}-\Delta_{S}^{+}$plane in the form of a line of QCPs, it is determined by the relevant direction at each point on the line of QCPs.

\section{B. $\epsilon_{d}$ expansion}

Next let us address the effects of CSB disorder within the framework of an $\epsilon_{d}$ expansion. In this method the RG flow equations become very complicated due to the ultraviolet divergent contribution arising from the class of the Feynman diagrams shown in Figs. 5(c) and 5(d), and it is challenging to decode the emergent quantum-critical phenomena. Thus we attempt to unearth critical properties by focusing on various coupling constant subspaces that remain closed under the $\mathrm{RG}$, at least to the leading order. Let us first focus on spin-orbit or axial magnetic disorder. The RG flow equations read

$$
\begin{aligned}
& \beta_{\Delta_{X}}=-\epsilon_{d} \Delta_{X}-\frac{2}{3} \Delta_{X}^{2}+2 \Delta_{X} \Delta_{A}, \\
& \beta_{\Delta_{A}}=-\epsilon_{d} \Delta_{A}+2 \Delta_{A}^{2}-6 \Delta_{A} \Delta_{X}+4 \Delta_{X}^{2},
\end{aligned}
$$

where $X=S O, A M$. Notice that even though the bare theory contains only spin-orbit or axial magnetic disorders, the CSP axial disorder gets generated, and in order to keep the RG flow equations closed, we need to include the latter from the outset. The coupled flow equations support one QCP, located at $\Delta_{X, *}=9 \epsilon_{d} / 10, \Delta_{A, *}=6 \epsilon_{d} / 5[30,42]$. The RG flow diagram is shown in Fig. 17(a), and the resulting phase diagram is displayed in Fig. 17(b). Note that QCP obtained in the absence of the CSB disorders, located at $\Delta_{A, *}=\epsilon_{d} / 2$, now becomes unstable in the presence of either spin-orbit or axial magnetic disorder, and a new QCP results from the competition between these two disorders, as mentioned above. This outcome, although is in contrast with our previously reported results obtained from $\epsilon_{m}$

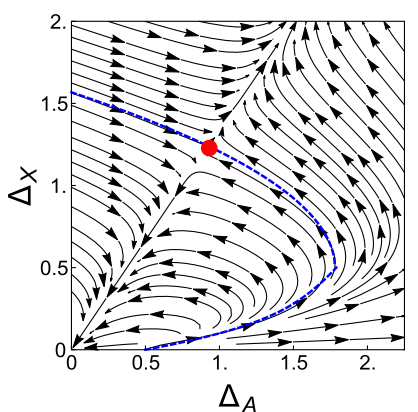

(a)

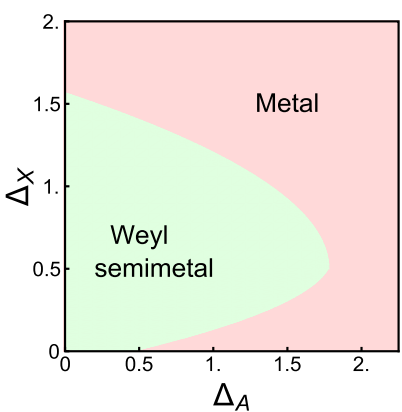

(b)
FIG. 17. (a) The renormalization-group flow diagram and (b) corresponding phase diagram in the $\Delta_{A}-\Delta_{X}$ plane obtained from $\epsilon_{d}$ expansion for $\epsilon_{d}=1$, where $X=S O, A M$. There exists only one QCP at $\Delta_{A}=6 \epsilon_{d} / 5, \Delta_{X}=9 \epsilon_{d} / 10$. The QCP at $\Delta_{A}=$ $\epsilon_{d} / 2$ in the absence of a CSB disorder now possesses two unstable directions. Note that a new critical point emerges from the competition between the chiral and nonchiral disorder $[30,42]$.

expansion, still shows some qualitative similarities, as we argue below. Notice that the DSE and CLE at the new QCP, shown in Fig. 17(a), are respectively given by

$$
z=1+\frac{9}{2} \epsilon_{d}+\mathcal{O}\left(\epsilon_{d}^{2}\right), \quad \nu^{-1}=\epsilon_{d}+\mathcal{O}\left(\epsilon_{d}^{2}\right) .
$$

As a result, the mean DOS at the QCP diverges as $Q(E) \sim$ $|E|^{-5 / 11}$ for $\epsilon_{d}=1$ or $d=3$, since $z>d$. Hence, both $\epsilon$ expansions give rise to diverging DOS at the QCP controlled via spin-orbit and axial magnetic disorder. Although the calculated values of DSE depend on the RG scheme, to the leading order they do not differ significantly; $z=6$ for $\epsilon_{m}=1$, and $z=11 / 2$ for $\epsilon_{d}=1$, while $\nu=1$ is independent of the RG scheme.

\section{Mass disorder}

We now discuss the role of mass disorder in WSMs. It should be noted that a WSM can be susceptible to two different types of mass disorder: (a) scalar mass disorder and (b) pseudoscalar mass disorder. Both of them break the chiral symmetry, but can be rotated into each other by the generator of the chiral symmetry $\gamma_{5}$. The flow equation for mass disorder within the framework of an $\epsilon$ expansion reads as

$$
\beta_{\Delta_{X}}=-\epsilon_{j} \Delta_{X}-\alpha_{j} \Delta_{X}^{2},
$$

for $X=S, P S$, where $\alpha_{m}=8 / 3$ and $\alpha_{d}=2, j=m, d$ corresponds to $\epsilon_{m}$ and $\epsilon_{d}$ expansions, respectively. Hence, by itself scalar or pseudoscalar mass disorder does not drive any WSM-metal QCP, at least within the leading order in $\epsilon$ expansions. In this regard, both $\epsilon_{m}$ and $\epsilon_{d}$ expansions yield an identical result. 
Finally, we discuss yet another interesting aspect of mass disorder, when it coexists with the axial one. The flow equations in the presence of these two disorders are

$\beta_{\Delta_{A}}=-\Delta_{A}\left[\epsilon_{j}-\tilde{\alpha}_{j} \Delta_{-}\right], \quad \beta_{\Delta_{X}}=-\Delta_{X}\left[\epsilon_{j}+\tilde{\alpha}_{j} \Delta_{-}\right]$,

for $X=S, P S$, where $\Delta_{-}=\Delta_{A}-\Delta_{X}, \tilde{\alpha}_{m}=8 / 3, \tilde{\alpha}_{d}=2$, and, respectively, $j=m, d$, corresponds to $\epsilon_{m}$ and $\epsilon_{d}$ expansions. These two flow equations support a line of QCPs, determined by

$$
\Delta_{A, *}=\frac{\epsilon_{j}}{\tilde{\alpha}_{j}}+2 \Delta_{X, *} \cdot
$$

The location of such a line of QCPs is regularization dependent (through $\tilde{\alpha}_{j}$ ), along which the DSE and CLE, given by

$$
z=1+\frac{\epsilon_{j}}{2}+2 \Delta_{S, *}, \quad \nu^{-1}=\epsilon_{j},
$$

are identical in both $\epsilon$-expansion schemes. Therefore, in a WSM with these two disorders the DSE continuously increases from $z=3 / 2$ in an unbounded fashion, while the CLE remains fixed. The numerical investigation of such an interesting possibility is left for a future work.

\section{Why is the chiral superuniversality so robust?}

Leaving aside the interesting possibilities of realizing such a line of QCPs with continuously varying critical exponents, perhaps the most urgent issue to be addressed is the following: Why does the disorder-driven WSM-metal QPT always display the same universality class, characterized by $\nu \approx 1$ and $z \approx 1.5$ ?

The answer to this question in the presence of intranode or chiral-symmetric disorders has already been provided in Sec. V. Note that the scaling dimension of any disorder coupling in a $d$-dimensional WSM is $\left[\Delta_{a}\right]=2 z-d$. But at all CSB disorder-driven QCPs, controlling the WSMmetal QPT, $z>d$ irrespective of the RG methodology. Therefore, even though the bare values of CSP disorders in lattice-based simulations are set to be zero, discussed in Sec VI B, they do get generated as we approach the Weyl points through the coarse-graining procedure. Ultimately the CSP disorder becomes relevant at CSB disorder-driven WSM-metal QCPs. As a result, the dirty system, even though it initially tends to flow toward the QCPs with $z>d$, described in this section, flows back toward the chiral-symmetric QCP or line of QCPs shown in Fig. 10(a). This is the reason why the WSM-metal QPTs are always characterized by CLE $\nu \approx 1$ and DSE $z \approx 1.5$ (within numerical accuracy), the characteristics of the proposed chiral superuniversality. The above argument is very generic and does not depend on the number of Weyl nodes. Therefore, in any lattice system, we expect
WSM-metal QPT to always belong to the chiral superuniversality. This outcome can be anchored from the RG calculation in the presence of all eight possible disorder couplings (since in a strong disorder regime all disorders get generated even if the bare coupling for some specific channel is set to be zero), as shown in Appendix G within the framework of both $\epsilon_{m}$ and $\epsilon_{d}$ expansions. Such analysis confirms that only the line of QCPs, defined through Eq. (36) or Eq. (39), and shown in Fig. 10(a), ultimately controls the quantum-critical behavior. Among all possible WSM-metal QCPs, we note that along the entire line of QCPs in the plane of regular and axial potential disorders, shown in Fig. 10, the DSE possesses the least (and constant) value. As a consequence, ADOS is smallest along this line of QCPs, which is thus expected to be robust against any perturbation. Therefore, we believe that the proposed notion of emergent superuniversality across such a line of QCPs in the chiral-symmetric hyperplane is nonperturbative in nature, which is further substantiated by our complementary numerical analysis, always yielding $z \approx 1.5$ and $\nu \approx 1$ (within numerical error bars); see Table I and Table II. This strongly supports the above argument in favor of chiral superuniversality under generic circumstances [85].

The specific tight-binding model we subscribe in this work (see Sec. II) also contains Wilson mass that bears higher gradient terms, such that $\tau_{3} b_{\perp}\left(k_{x}^{2}+k_{y}^{2}\right)$, with $b_{\perp}=$ $t_{0} a^{2} / 2$. The scaling dimension of such an operator is $\left[b_{\perp}\right]=z-2$. Hence, the higher gradient terms are irrelevant at the clean WSM fixed point $\left(\left[b_{\perp}\right]=-1\right)$ as well as at the chiral-symmetric line of QCPs $\left(\left[b_{\perp}\right]=-1 / 2\right)$, but become relevant at pure CSB disorder-driven QCPs (since $z>d>2$ ). This is also the reason why chiral superuniversality is such a generic and utmost stable situation.

Furthermore, we also show that the chiral superuniversality does not depend on the choice of disorder distribution. For example, in Appendix $\mathrm{F}$ we perform similar analysis of average DOS in the presence of correlated potential disorder that by construction significantly suppresses the intervalley scattering (at least when disorder is sufficiently weak). However, the universality class of the WSM-metal QPT (characterized by $z$ and $\nu$ ) remains unchanged (within numerical accuracy) by the profile of the distribution function. This observation should further strengthen the proposed scenario of emergent superuniversality (insensitive to the nature of disorder and its distribution) across the WSM-metal QPT.

Nevertheless, we believe pure CSB disorder-driven QCPs (with $z>d$ ) can in principle be realized in a numerical simulation performed in momentum space, where forward or intranode or CSP scattering processes can be suppressed deliberately and higher gradient terms can be avoided completely. Such an analysis is an interesting exercise of a pure academic interest, and we leave it for a future investigation. 


\section{QUANTUM-CRITICAL SCALING OF PHYSICAL OBSERVABLES}

As demonstrated in the previous two sections, QPT from a WSM to a diffusive metal can be driven by different types of elastic scatters, and the critical exponents are remarkably independent of the actual nature of randomness. We here highlight how these exponents can affect the scaling behavior of measurable quantities as the Weyl material undergoes this QPT [86].

\section{A. Residue of quasiparticle pole}

As the WSM-metal QCP is approached from the semimetallic phase, the residue of the quasiparticle pole vanishes, and beyond the critical strength of disorder Weyl fermions cease to exist as sharp quasiparticle excitations, similar to the situation for two-dimensional Dirac fermion-Mott insulator QPT in the presence of a strong Hubbard interaction $[87,88]$. The residue of quasiparticle pole $(Z)$ vanishes as

$$
Z \sim\left(\frac{\Delta_{*}-\Delta}{\Delta_{*}}\right)^{\nu \eta_{\Psi}} \equiv \delta^{\nu \eta_{\Psi}}
$$

where $\eta_{\Psi}$ is the fermionic anomalous dimension at the critical point located at the disorder strength $\Delta=\Delta_{*}$. Within the framework of an $\epsilon_{d}$ expansion, $\eta_{\Psi}=0$ to the leading order in $\epsilon_{d}$, and one needs to account for two-loop diagrams to obtain finite $\eta_{\Psi}$. In contrast, in the $\epsilon_{m}$ expansion we obtain nontrivial fermionic anomalous dimension even to the one-loop order, and $\eta_{\psi} \sim \epsilon$, as shown in Eq. (27). Therefore, at the WSM-metal QCP, the quasiparticle spectrum displays a branch cut and the critical point represents a strongly coupled non-Fermi liquid. Alternatively, the residue of quasiparticle pole plays the role of a bona fide order parameter on the semimetallic side. It is worth mentioning that the disappearance of residue of quasiparticle pole has recently been tracked in quantum Monte Carlo simulations for the Hubbard model in a two-dimensional honeycomb lattice [88], and we can expect that future numerical work can verify our proposed scaling form in Eq. (51) across the disorder-driven WSMmetal QPTs. The Fermi velocity scales as $v \sim|\delta|^{\nu(z-1)}$, and since $z>1$ at the QCP or the quantum-critical line, the Fermi velocity vanishes at the transition to the metallic phase. A subsequent numerical work has demonstrated the suppression of residue of quasiparticle pole [89].

\section{B. Average density of states}

The most widely studied physical quantity in numerical simulations across the WSM-metal QPT is the average DOS $[52,54,56,57,59,63]$. Since throughout the paper we have already extensively used the average DOS to characterize phases, for the sake of completeness we here review only its salient features. We can infer the scaling form of the average DOS in the thermodynamic limit $L \rightarrow \infty$ in different phases by using its scaling function [see Eq. (14)]. In the quantum-critical regime $\varrho(E)$ should be independent of $\delta$, yielding $\varrho_{Q}(E) \sim E^{d / z-1}$. Inside the WSM phase, the average DOS scales as $\varrho_{W}(E) \sim$ $\delta^{(1-z) d \nu}|E|^{2}$. In the metallic phase, average DOS at zero energy is finite and scales as $\varrho(0) \sim \delta^{(d-z) \nu}$. From the quoted values of DSE and CLE, it is straightforward to find the scaling of average DOS in these three regimes of the phase diagram in a dirty WSM, which we have used in the numerical analysis of this observable in the previous sections.

\section{Conductivity}

The optical conductivity $(\sigma)$ at $T=0$ can as well serve as an order parameter across the WSM-metal QPT, and assumes the following scaling ansatz for frequency $(\Omega)$ much smaller than the bandwidth [43],

$$
\sigma(\Omega)=\delta^{\nu(d-2)} \mathcal{G}\left(\Omega \delta^{-\nu z}\right)
$$

where $\mathcal{G}$ is yet another unknown universal scaling function. This scaling form remains operative even at finite temperature as long as $\Omega \gg T$, i.e., in the collisionless regime. In the collision-dominated regime at $T \gg \Omega$, the dc conductivity also assumes a similar scaling form as in Eq. (52), upon replacing the frequency $(\Omega)$ by temperature $(T)$ $[37,53,90]$. In the WSM side of the transition, the optical conductivity vanishes linearly with $\Omega$ and scales as $\sigma_{W}(\Omega) \sim \delta^{\nu(1-z)(d-2)} \Omega^{d-2}$. Inside the critical regime the optical conductivity scales as $\sigma_{Q}(\Omega) \sim \Omega^{(d-2) / z}$. In the presence of strong CSP disorder $z \approx 3 / 2$, and the optical conductivity inside the quantum-critical regime thus vanishes as $\sigma_{Q}(\Omega) \sim \Omega^{2 / 3}$. Since for nonchiral disorder the DSE is typically much bigger than in the presence of a chiral-symmetric one, the optical conductivity vanishes with a weaker power as $\Omega \rightarrow 0$ when the system is still dominated by CSB disorder before CSP disorder takes over. Hence, in this regime the system becomes more metallic in the presence of CSB disorder than with only CSP disorder. Inside the metallic phase, the optical conductivity becomes finite and scales as $\sigma_{M}(0) \sim \delta^{\nu(d-2)}$ as $\Omega \rightarrow 0$. Within the leading-order $\epsilon_{m}$ or $\epsilon_{d}$ expansions, the conductivity of the metal is therefore always independent of the actual nature of elastic scatterers, since $\nu^{-1}=\epsilon_{m}$ or $\epsilon_{d}$, and $\epsilon_{m}=1, \epsilon_{d}=1$. Otherwise, weak disorder (such as potential) causes enhancement of optical conductivity without altering $\sigma \sim \Omega$ scaling [43] (see also Appendix $\mathrm{H}$ for a simple derivation).

\section{Specific heat}

The specific heat $\left(C_{v}\right)$ also displays distinct scaling behavior in three regimes of the phase diagram of a dirty 
WSM. The scaling of specific heat at temperature much smaller than bandwidth follows the ansatz [36]

$$
C_{V}(T)=\frac{T^{d / z}}{v^{d}} \mathcal{H}\left(T \delta^{-\nu z}\right),
$$

where $\mathcal{H}$ is also an unknown universal scaling function. In the WSM phase, $\mathcal{H}(x) \sim x^{d(z-1) / z}$ and the specific heat scales as $C_{V} \sim \delta^{d \nu(1-z)} T^{d}$, so that we recover $T^{3}$ dependence for the three-dimensional Weyl fermion. Inside the metallic phase, $\mathcal{H}(x) \sim x^{1-d / z}$, yielding $C_{V} \sim \delta^{\nu(d-z)} T$, and we obtain $T$-linear specific heat, similar to the situation in Fermi liquids. By contrast, inside the critical regime $H(x) \sim x^{0}$, yielding $C_{V} \sim T^{3 / z}$. Therefore, the specific heat, analogous to the conductivity, displays distinct power-law dependence on temperature inside the quantum-critical regime depending on the dominant source of disorder, while its scaling inside the WSM and metallic phases is insensitive to the nature of random impurities. Hence, the scaling of specific heat can be used to extract the extent of the critical regime and crossover boundaries among different phases of a dirty Weyl system at finite temperature [56].

\section{E. Mean-free path}

The quasiparticle mean-free path $(\mathcal{L})$ also follows the critical scaling

$$
[\mathcal{L}(E)]^{-1}=\delta^{\nu} \mathcal{J}\left(E \delta^{-\nu z}\right)
$$

where $\mathcal{J}$ is a universal, but unknown, scaling function, with energy much smaller than bandwidth. At the QCP $(\delta=0)$ the mean-free path should be independent of $\delta$, implying $\mathcal{J}(x) \sim x^{-1 / z}$. Therefore, inside the quantum-critical fan, the mean-free path at zero energy diverges as $\mathcal{L}(E) \sim$ $E^{-1 / z}$. In the metallic phase, $\mathcal{J}(x) \sim x^{0}$ as $x \rightarrow 0$, leading to finite mean-free path at zero energy, and $\mathcal{L}(0) \sim \delta^{-\nu}$. On the other hand, in the WSM phase, the mean-free path $\mathcal{L}_{W}(E) \sim \delta^{\nu(z-1)} E^{-1}$, as $E \rightarrow 0$. Since at all disorder-driven QCPs $z>1, \mathcal{L}_{W}(E)$ decreases with increasing disorder, indicating propensity toward the onset of a metallicity in the system.

\section{F. Grüneisen parameter}

Yet another directly measurable quantity is the Grüneisen parameter, defined as $\gamma=\alpha / C_{P}$, where $\alpha$ is the thermal expansion parameter, and $C_{P}$ is the specific heat measured at constant pressure. The Grüneisen ratio in the WSM phase is $\gamma_{W} \sim T^{-4}$, while inside the critical regime $\gamma_{Q} \sim T^{-(1+d / z)}$. Inside the metallic phase $\gamma_{M} \sim T^{-2}$. Therefore, the Grüneisen parameter displays distinct power-law behavior in three different phases of a dirty WSM.

Fascinating scaling behavior can also be observed for the magnetic Grüneisen ratio, defined as $\Gamma_{H}=(\partial M / \partial T)_{H} /$ $C_{H}$, where $M \propto H$ is magnetization, $C_{H}$ is the molar specific heat, and $H$ is the magnetic field strength. In the presence of sufficiently weak randomness when Landau quantization is sharp $\left(\omega_{c} \tau \gg 1\right.$, where $\omega_{c}$ is cyclotron frequency and $\tau$ is scattering lifetime) and it dominates over the Zeeman coupling, leading to $\Gamma_{H} \sim T^{-4 / z}$. On the other hand, in the presence of strong elastic scattering, when $\omega_{c} \tau \ll 1$, the Landau levels are sufficiently broadened and the dominant energy scale is set by Zeeman coupling, yielding $\Gamma_{H} \sim T^{-2}$, which is independent of dimensionality (d) or DSE (z). Therefore, for a fixed weak magnetic field, as the strength of impurities is gradually increased, the magnetic Grüneisen ratio should display a smooth crossover from $T^{-4}$ to $T^{-2}$ dependence. Note that such a crossover will take place even before the system enters the quantum-critical regime and will persist in the metallic regime as well, since elastic scattering is strong in these two phases.

\section{ANDERSON TRANSITION}

As a final topic, we discuss the Anderson transition (AT) of a disordered diffusive Weyl metal at stronger strength of disorder. For the sake of simplicity we here focus only on the effects of random charge impurities. Possible AT in the presence of all other disorder is left for a future investigation. To study the AT we compare three different types of DOS, namely ADOS $\left[\varrho_{a}(E)\right]$, local DOS (LDOS) $\left[\varrho_{L}(E)\right]$ and typical DOS (TDOS) $\left[\varrho_{t}(E)\right]$, respectively defined as [72,77]

$$
\begin{gathered}
\varrho_{a}(E)=\left\langle\frac{1}{2 L^{3}} \sum_{i=1}^{L^{3}} \sum_{\alpha=1}^{2} \delta\left(E-E_{i, \alpha}\right)\right\rangle, \\
\varrho_{L}^{i, \alpha}(E)=\sum_{k, \beta}|\langle k, \beta \mid i, \alpha\rangle|^{2} \delta\left(E-E_{k, \beta}\right), \\
\varrho_{t}(E)=\exp \left[\frac{1}{2 N_{s}} \sum_{j=1}^{N_{s}} \sum_{\alpha=1}^{2}\left\langle\log \varrho_{L}^{i, \alpha}(E)\right\rangle\right] .
\end{gathered}
$$

Here, $L^{3}$ is the system size, and $|i, \alpha\rangle$ is the eigenstate with site index $i$ and orbital index $\alpha(=1,2)$ at energy $E_{i, \alpha}$. As previously discussed, ADOS is a self-averaging quantity, so to minimize statistical fluctuations we only extract the disorder-averaged smoothened data, which we carry out by computing $N_{m}=1024$ Chebyshev moments and performing disorder average over 20 random disorder realizations. On the other hand, LDOS and TDOS are not self-averaging quantities. Therefore, numerical extraction of TDOS is extremely demanding, for which we compute $N_{m}=8192$ moments and perform disorder average over 100 random disorder realizations to construct the TDOS. To further suppress statistical fluctuations in TDOS we average over a small cube of size $N_{s}=L_{s}^{3} \ll L^{3}$, and we here take $L_{s}=4$. Such averaging is justified since translational 


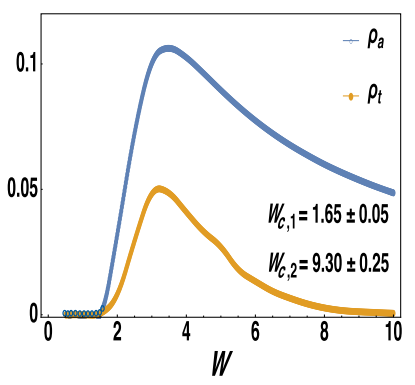

(a)

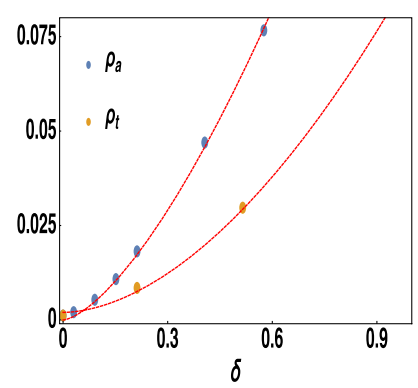

(b)
FIG. 18. (a) Scaling of average $\left[\varrho_{a}(0)\right]$ and typical $\left[\varrho_{t}(0)\right]$ density of states at zero energy as a function of disorder strength. The Weyl semimetal-metal and metal-Anderson insulator quantum phase transitions, respectively, take place at $W_{c, 1}=1.65 \pm$ 0.05 and $W_{c, 2}=9.30 \pm 0.25$. (b) Scaling of these two quantities as a function of $\delta=\left(W-W_{c, 1}\right) / W_{c, 1}$, yielding corresponding order-parameter exponents [defined in Eq. (58)] $\beta_{a}=1.50 \pm$ 0.05 and $\beta_{t}=1.80 \pm 0.20$.

symmetry gets restored after disorder averaging has been performed.

The scaling of ADOS and TDOS over a wide range of disorder strength is shown in Fig. 18(a). Note that in the WSM phase both ADOS and TDOS at zero energy are pinned to zero, which then become finite across the WSMmetal QPT at $W_{c, 1}=1.65 \pm 0.05$. Therefore, either ADOS or TDOS can be identified as a bona fide order parameter to pin the WSM-metal QCP. Respectively these two quantities scale as

$\varrho_{a}(0) \sim\left(\frac{W-W_{c, 1}}{W_{c, 1}}\right)^{\beta_{a}}, \quad \varrho_{t}(0) \sim\left(\frac{W-W_{c, 1}}{W_{c, 1}}\right)^{\beta_{t}}$,

near the WSM-metal QCP, with

$$
\beta_{a}=1.50 \pm 0.05, \quad \beta_{t}=1.80 \pm 0.20,
$$

as shown in Fig. 18(b). Even though the numerical error bar for $\beta_{t}$ is quite large, in general, we expect it to be different from $\beta_{a}$, as their difference, $\Delta \beta=\beta_{t}-\beta$, is intimately tied with the multifractal dimension of the wave function across a disorder-driven QPT [91-94]. However, more precise determination of $\beta_{t}$ requires additional extensive numerical simulation. Therefore, we leave this issue as a subject for a future investigation.

Inside the compressible diffusive metallic phase these two quantities increase monotonically and follow each other up to a moderate strength of disorder $W_{*} \approx 3.5$. Upon further increasing strength of disorder the TDOS smoothly vanishes around $W_{c, 2}=9.30 \pm 0.25$. Therefore, a metalinsulator transition (MIT) takes place at $W=W_{c, 2}$, commonly known as AT. Note that the ADOS decreases monotonically across the AT, but remains noncritical, as shown in Fig. 18(a). In Fig. 19(a), we present the scaling of TDOS with the number of Chebyshev moments $\left(N_{m}\right)$.

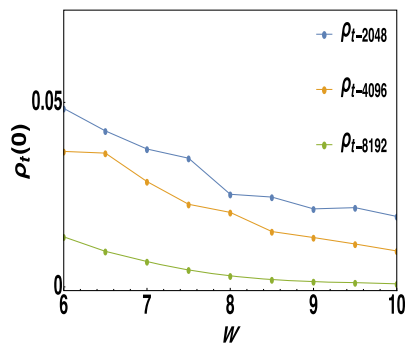

(a)

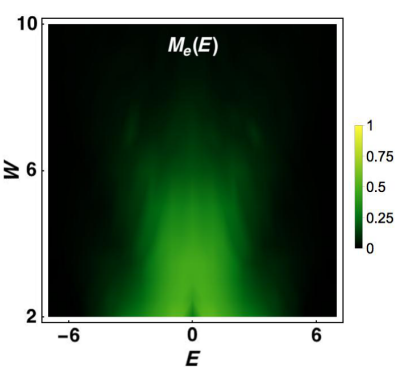

(b)
FIG. 19. (a) Scaling of typical density of states at zero energy $\varrho_{t}(0)$ for disorder $(W)$ within the range $6.0 \leq W \leq 10.0$ with the number of Chebyshev moments $N_{m}$. We here compute $\varrho_{t}(0)$ for $N_{m}=2048$, 4096, and 8192. From the scaling of $\varrho_{t}(0)$ versus $N_{m}$, we find that metal-insulator Anderson transition takes palce at $W_{c, 2}=9.30$ in the $N_{m} \rightarrow \infty$ limit. (b) Mobility edge $M_{e}(E)$ [defined in Eq. (61)] as a function of energy $(E)$ and disorder $(W)$. Respectively, the green and the dark regions accommodate extended or metallic and localized states. Scale of $M_{e}(E)$ is shown in the legend. Here the system size is $L=80$.

We explicitly compute TDOS from moderate to strong disorder regime $(6 \leq W \leq 10)$, in close vicinity of the AT, for $N_{m}=2048,4096$, and 8192. From the scaling of $\varrho_{t}(0)$ versus $N_{m}$ we conclude that AT [identified with $\varrho_{t}(0) \rightarrow 0$ ] takes place around $W_{c, 2}=9.30$ in the $N_{m} \rightarrow \infty$ limit. Therefore, we can conclude that a three-dimensional diffusive Weyl metal is a stable phase of matter for moderately strong disorder, which ultimately undergoes a QPT into the Anderson insulator phase for sufficiently strong disorder. Across the AT the TDOS at zero energy display single-parameter scaling,

$$
\varrho_{t}(0) \sim\left(\frac{W-W_{c, 2}}{W_{c, 2}}\right)^{\beta},
$$

with $\beta=1.5 \pm 0.15$. Critical scaling of typical DOS across the Anderson transition strongly suggests that wave functions at the Anderson critical point become multifractal in nature [77]. A detailed analysis of a multifractal spectrum requires the notion of exact wave function, which is numerically very time consuming. Nevertheless, analysis of the multifractal nature of wave functions in a timereversal symmetry-breaking topological metal is a problem of fundamental importance, which we leave for future investigation.

Recall that for weak disorder ADOS $\varrho_{a}(E) \sim|E|^{2}$ and around the WSM-metal QCP it scales as $\varrho(E) \sim|E|$. Inside the metallic phase $\varrho_{a}(0)$ is finite. In Fig. 20(a), we show that within the range of disorder strength 0.50 (weak) $\leq$ $W \leq 3.5$ (moderate), the TDOS also displays the same scaling behavior as ADOS. This observation confirms that TDOS can also be subscribed as a bona fide order parameter across the WSM-metal QPT. On the other hand, for strong enough disorder the TDOS $\varrho_{t}(E)$ decreases 


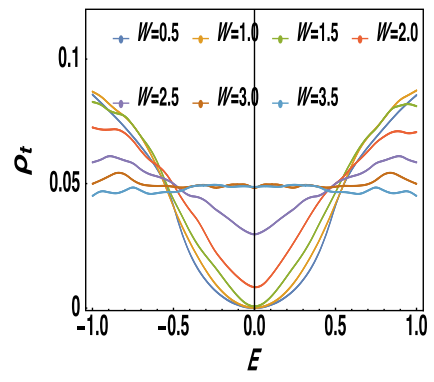

(a)

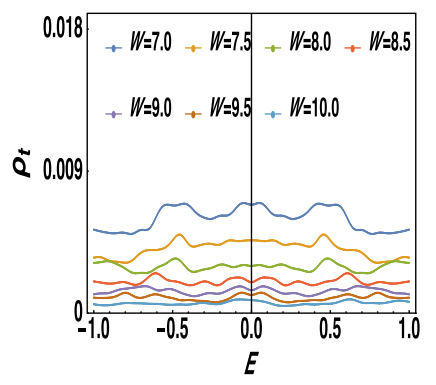

(b)
FIG. 20. (a) Scaling of typical density of states $\left[\varrho_{t}(E)\right]$ versus energy $(E)$ from weak to moderately strong disorder, showing that $\varrho_{T}(E) \sim|E|^{2}$ of weak disorder. Also note that $\varrho_{t}(E) \sim|E|$ around $W=W_{c, 1} \approx 1.65$, and inside the metallic phase $\varrho_{t}(0)$ is finite. These features are qualitatively similar to the ones for the average density of states [see Fig. 3]. (b) Scaling of $\varrho_{t}(E)$ versus $E$ for stronger disorder (close proximity to the Anderson metalinsulator transition), showing that $\varrho(0)$ smoothly vanishes across the Anderson transition, and remains pinned at zero inside an Anderson insulator. Here we compute 8192 Chebyshev moments to construct $\varrho_{t}(E)$ in a system with linear dimension $L=80$.

monotonically for any energy $E$, and ultimately $\varrho_{t}(0)$ becomes zero across the AT. Therefore, TDOS can serve as the order parameter across all possible disorder-driven QPTs considered here.

Finally, we focus on the evolution of the location of the mobility edge in a dirty Weyl metal as a function of disorder strength by numerically computing the mobility edge, defined as

$$
M_{e}(E)=\frac{\varrho_{t}(E)}{\varrho_{a}(E)} .
$$

In particular, the mobility edge defines the boundary between the extended and localized states, and we here focus on this quantity in the strong disorder regime $W \geq 2>W_{c, 1}$. The results are shown in Fig. 19(b). For weak disorder the mobility edge resides at high energy, indicating the metallic nature of a moderately dirty Weyl system. However, the mobility edge progressively slides down toward smaller energy with increasing randomness in the system. Finally, across the AT the mobility edge comes down to zero energy, indicating that all states inside the Anderson insulator are localized. Notice that the shape of the mobility edge is quite distinct in a Weyl metal in comparison to its counterpart in conventional metal [95], which, however, can solely be attributed to the linear dispersion of Weyl quasiparticles in the clean system.

\section{SUMMARY AND DISCUSSION}

In this paper, we study the role of generic disorder in a Weyl semimetal, by considering its simplest realization, composed of only two Weyl nodes. When the system resides in the proximity of semimetal-insulator quantum phase transition, the generalized Harris criterion suggests that such a critical point is stable in the presence of weak but generic disorder. By contrast, a multicritical point appears in the phase diagram for strong disorder, where the Weyl semimetal, an insulator, and a metallic phase meet. Within the framework of an appropriate $\epsilon$ expansion we show that, to the leading order, the critical exponents at such multicritical point are (i) dynamic scaling exponent $z=1+\epsilon_{n} / 2$ and (ii) correlation length exponent $\nu=1 / \epsilon_{n}$ that controls the relevance of disorder coupling, where $\epsilon_{n}=1 / 2$ for a physical system. These findings are in good agreement with the ones obtained numerically, yielding $\nu=1.98 \pm 0.10$ and $z=1.26 \pm 0.05$.

On the other hand, when the system is deep inside the Weyl semimetal phase, we have shown that the continuous global chiral U(1) symmetry plays a fundamental rule in classifying the disorder-driven Weyl semimetal-metal quantum phase transitions. The simplest realization of a Weyl semimetal is susceptible to eight types of disorder, among which only four preserve such chiral symmetry. Using two different $\epsilon$ expansions, we show that the chiralsymmetric disorder-driven semimetal-metal transition takes place through either a quantum-critical point or a line of quantum-critical points. Irrespective of details, the critical exponents to the leading order in $\epsilon$ expansions are given by $z=1+\epsilon / 2+\mathcal{O}\left(\epsilon^{2}\right)$ and $\nu=\epsilon^{-1}+\mathcal{O}\left(\epsilon^{2}\right)$, and $\epsilon=1$ corresponds to the physical situation. Even though these exponents can receive higher-order corrections $\mathcal{O}\left(\epsilon^{2}\right)$, presently there is no known route to compute them in a controlled fashion beyond the leading order in $\epsilon$. Such a unique set of exponents in the presence of generic chiralsymmetric disorder gives birth to an emergent chiral superuniversality across the Weyl semimetal-metal quantum phase transition.

Furthermore, we have performed a thorough numerical analysis of average density of states in Weyl semimetals with chiral-symmetric disorder. The emergence of chiral superuniversality has been demonstrated through numerical analysis of average density of states near zero energy. We show that for any such disorder, Weyl semimetal undergoes a continuous quantum phase transition into a diffusive metallic phase. Within the numerical accuracy, we find that across this transition $z \approx 1.5$ and $\nu \approx 1$, in excellent agreement with our field-theoretic predictions obtained from leading-order $\epsilon$ expansions (see Table I for comparison). The quality as well as reliability of our numerical analysis have been anchored through two completely different types of high-quality data collapses, shown in Fig. 13, in the entire phase diagram of a dirty Weyl semimetal for all possible chiral disorder.

For chiral-symmetry-breaking disorder, the Weyl semimetal-metal quantum phase transition also takes place through a critical point or a line of critical points, but the critical exponents are significantly different from the ones reported in the presence of chiral disorder. Even 
though the critical exponents across such a semimetalmetal transition turn out to be slightly dependent on the renormalization-group scheme, we always find $z>d$ and $\nu=1 / \epsilon$ from leading-order $\epsilon$ expansions. Consequently, all chiral-symmetric or intranode disorder (as well as higher gradient terms that are inevitably present in a lattice) become relevant at such a putative line of critical points. As a result, internode disorder-driven semimetal-metal phase transition is ultimately always governed by the chiral-symmetric disorder, yielding $\nu \approx 1$ and $z \approx 3 / 2$, characteristic of chiral superuniversality. We anchor these outcomes by numerically extracting the scaling of average density of states in the presence of internode disorder, and the results are shown in Table II, Fig. 3 (lower panel), and Fig. 14.

Even though we promote such a classification scheme in a Weyl semimetal with only two nodes, our prescription can easily be generalized to Weyl systems with multiple flavors, as well as topological Dirac semimetals with bona fide time-reversal symmetry that has recently been found in $\mathrm{Cd}_{2} \mathrm{As}_{3}$ [96] and $\mathrm{Na}_{3} \mathrm{Bi}$ [97] and the ones at the quantumcritical point residing between two topologically distinct insulating vacua.

We here mention that $\epsilon_{d}$ expansion can be problematic beyond the leading order in $\epsilon_{d}$, since the contribution from Figs. 5(c) and 5(d) and their higher-loop cousins are typically ultraviolet divergent and one loses the orderby-order control over the perturbative calculation [40,45]. For example, it was shown in Refs. $[40,45]$ that to the order $\epsilon_{d}^{3}$ the correlation length exponent is (see also Ref. [41])

$$
\nu^{-1}=\epsilon_{d}+\frac{1}{2} \epsilon_{d}^{2}+\frac{3}{8} \epsilon_{d}^{3},
$$

respectively yielding $\nu^{(2)}=0.66$ and $\nu^{(3)}=0.53$ to the two- and three-loop order for $\epsilon_{d}=1$. Upon implementing the Padé resummation [note that $\nu^{(2)}=0.5$ is obtained from Padé [1|1] resummation, while $\nu^{(3)}=0.33$ and 0.375 are, respectively, obtained from Padé [2|1] and Padé [1|2] resummation; see Refs. [40,45] for details], we obtain $\nu^{(2)}=0.5$ and $\nu^{(3)}=0.33$ or 0.375 (both being smaller than the mean-field value of $\nu=1 / 2)$. Hence, $\epsilon_{d}$ expansion runs into the serious problem of convergence beyond the leading order. Such a class of diagrams is, however, ultraviolet finite and thus does not contribute to renormalization-group flow equations in the $\epsilon_{m}$ expansion scheme (see Appendix C 3). We, therefore, believe that higher-order perturbation theory within the framework of an $\epsilon_{m}$ expansion should be more controlled. Explicit higher-order calculation in $\epsilon_{m}$ expansion and its corroboration with a newly proposed nonperturbative approach combined with the functional renormalization-group analysis [98] is, however, left as a challenging interesting problem for future investigation. Nonetheless, we note that leading-order $\epsilon_{d}$ and $\epsilon_{m}$ expansions, as well as the functional renormalization-group approach from Ref. [98], yield identical values for the critical exponents, namely, $z=3 / 2$ and $\nu=1$.

In addition to the Weyl semimetal-metal quantum phase transition, we also establish that a compressible Weyl metal undergoes a subsequent transition at stronger disorder into an Anderson insulator. We track the typical density of states to pin the onset of such an insulating phase that only accommodates localized states. In particular, we show that across the Weyl metal-insulator transition the typical density of states at zero energy $\left[\varrho_{t}(0)\right]$ smoothly vanishes, and thus serves as a bona fide order parameter, while the average density of states remains noncritical across this transition. In addition, we also find that $\varrho_{t}(0)$ remains pinned in the Weyl semimetal phase and becomes finite in the metallic phase. Therefore, typical density of states at zero energy serves as a unified order parameter across all possible disorder-driven quantum phase transitions in a Weyl semimetal.

Finally, we comment on some nonperturbative effects of disorder in Weyl semimetals, such as puddles [99], Lishiftz tail [100], and rare-region states and Griffiths physics $[34,61]$. Puddles are inevitable in real materials as there are always density fluctuations that locally shift the chemical potential away from the Weyl nodes, while maintaining the overall charge neutrality of the system. In addition, the presence of disorder can also support quasilocalized rare states at zero energy even for subcritical strength of disorder [34,61]. Although such effects are important and interesting, they possibly do not affect the quantum-critical behavior. Also, the presence of finite average DOS close to zero energy for subcritical disorder does not necessarily imply a finite typical DOS at zero energy $\left[\varrho_{t}(0)\right]$ and a finite dc conductivity as $T \rightarrow 0$, the hallmark signatures of a metal. By contrast, we find that $\varrho_{t}(0)$ remains pinned to zero for weak enough disorder; see Fig. 18(a). In addition, whether generic disorder (inter- and intranode) accommodates rare regions remains to be examined. Furthermore, it is not clear if the rare states can survive when they hybridize with nonrare or critical states, residing close in energy. On the other hand, a recent numerical work has demonstrated that such nonperturbative effects can be systematically suppressed with a suitable choice of the distribution of disorder, while the critical properties across the Weyl semimetal-metal quantum phase transition remain almost unchanged [63]. Therefore, rare and critical excitations appear to be decoupled from each other (based on present numerical evidence) and these effects do not alter any physical outcome we report in this paper.

\section{ACKNOWLEDGMENTS}

B. R. was supported by NSF-JQI-PFC and LPS-MPOCMTC, and partially from Welch Foundation Grant No. C1809 and NSF CAREER Grant No. DMR- 1552327 of 
Matthew S. Foster (Rice University). We thank Sankar Das Sarma, Matthew Foster, Pallab Goswami, and Soumya Bera for useful discussions. B. R. and R.-J. S. are thankful to Nordita, for hospitality during the workshop "From Quantum Field Theories to Numerical Methods," where part of this work was finalized.

\section{APPENDIX A: GENERALIZED HARRIS CRITERION AT WSM-INSULATOR QCP}

In this appendix, we present a generalization of the Harris criterion applicable near the clean WSM-insulator QCP. Let us first consider a generalized version of the Hamiltonian from Eq. (6) describing the gapless excitations residing at general WSM-insulator QCP [80],

$$
\begin{aligned}
\hat{H}_{Q}^{c}(\mathbf{k}, \Delta)= & \alpha_{c}\left[\sigma_{1} k_{\perp}^{c} \cos \left(c \phi_{\mathbf{k}}\right)+\sigma_{2} k_{\perp}^{c} \sin \left(c \phi_{\mathbf{k}}\right)\right] \\
& +\sigma_{3}\left(b k_{3}^{2}-\Delta\right),
\end{aligned}
$$

where $k_{\perp}=\sqrt{k_{x}^{2}+k_{y}^{2}}$ and $\phi_{k}=\tan ^{-1}\left(k_{y} / k_{x}\right)$. The above Hamiltonain for any value of $\Delta$ possesses the same symmetry, but describes two distinct phases: (i) a band insulator for $\Delta<0$ and (ii) WSM for $\Delta>0$, with $c$ representing the monopole charge of the Weyl nodes. Respectively, for $c=1,2$, and 3, single, double, and triple WSMs are realized in a crystalline environment [101-103]. The effective dimensionality $\left(d_{*}\right)$ of such critical semimetallic phase can be found from the corresponding imaginary time Euclidean action,

$S^{c}=\int d \tau d^{2} x_{\perp} d x_{3} \psi^{\dagger}\left[\partial_{\tau}+\hat{H}_{Q}^{c}(\mathbf{k} \rightarrow-i \nabla, \Delta)\right] \psi$,

where $\psi$ is a two-component spinor, describing the critical excitations residing at the WSM-insulator QCP. All parameters, such as $\alpha_{c}$ and $b$, remain invariant under the rescaling of space-time (imaginary) coordinates according to $\tau \rightarrow e^{l} \tau,(x, y) \rightarrow e^{l / c}(x, y), x_{3} \rightarrow e^{l / 2} x_{3}$, when accompanied by the field normalization $\psi \rightarrow Z_{\psi}^{1 / 2} \psi$, where $Z_{\psi}=\exp \left\{\left[-(2 / c)+\frac{1}{2}\right] l\right\} \equiv \exp \left[-d_{*} l\right]$. The spatial measure $d^{2} x_{\perp} d x_{3} \rightarrow e^{d_{*} l} d^{2} x_{\perp} d x_{3}$, where $d_{*}=\left[(2 / c)+\frac{1}{2}\right]$ is the effective dimensionality of the system under the rescaling of spatial coordinates. Note that $\Delta$ in Eq. (A1) is the tuning (relevant) parameter at the WSM-insulator QCP, and the scaling dimension of $\Delta$, denoted by $[\Delta]$, is tied with the CLE $(\nu)$ at this QCP, according to $\nu^{-1}=[\Delta]=1$. The stability of the clean WSM-insulator QCP against mass disorder [denoted by $V_{z}(\mathbf{x})$ in Eq. (8)] can be assessed from the generalized Harris criterion, suggesting that such QCP is stable against mass disorder when

$$
\nu>\frac{2}{d_{*}}=\frac{4 c}{4+c} .
$$

Therefore, only the single ( $c=1)$ WSM-insulator QCP is stable against sufficiently weak mass or bond disorder. Furthermore, the stability of the WSM-insulator QCP in the presence of generic disorder, which appears similar to $V_{z}(\mathbf{x})$ in Eq. (8), can be established from the generalized Harris criterion [see Eq. (A3)]. Hence, a single WSMinsulator QCP is guaranteed to be stable against generic disorder. In this regard a comment is due. Our derivation of generalized Harris criterion differs from the original one in Ref. [27], where $d_{*}$ is replaced by the physical dimensionality of the system $(d)$ and the CLE $\nu$ varies depending on the nature of the phase transition. On the other hand, within the framework of anisotropic scaling of spatial coordinates, we always find $\nu=1$, but actual spatial dimension gets replaced by an effective dimensionality of the system $\left(d_{*}\right)$ under the process of coarse graining. We believe that these two methods are complementary to each other.

\section{APPENDIX B: RG ANALYSIS NEAR WSM-INSULATOR QCP}

In this appendix, we provide technical details of the RG calculations near the WSM-insulator QPT with disorder. First, we show the effects of subleading divergences in the RG flow equations within the $\epsilon_{n}$ expansion introduced in Sec. III and its consequences (see Sec. B 1). Next, we display the perturbative analysis of disorder near the WSMinsulator in an expansion about the lower critical dimension of the theory (see Sec. B 2).

\section{1. $\epsilon_{n}$ expansion}

Within the framework of $\epsilon_{n}$ expansion, discussed in Sec. III, after integrating out the fast Fourier modes within the Wilsonian shell $\Lambda e^{-l}<k_{\perp}<\Lambda$ and $0<k_{3}^{2}<\infty$ and accounting for subleading ultraviolet divergences, the RG flow equations read

$$
\begin{aligned}
\beta_{X}= & -X\left(\Delta_{0}+2 \Delta_{\perp}+\Delta_{z}\right)\left[h_{1}(n)+h_{2}(n)\right]=(1-z) X, \\
\beta_{\Delta}= & \Delta+\left\{\Delta\left[h_{1}(n)+h_{2}(n)\right]-h_{3}(n)\right\}\left(\Delta_{0}-2 \Delta_{\perp}+\Delta_{z}\right), \\
\beta_{\Delta_{0}}= & -\epsilon_{n} \Delta_{0}+2 \Delta_{0}\left(\Delta_{0}+2 \Delta_{\perp}+\Delta_{z}\right)\left[h_{1}(n)+h_{2}(n)\right] \\
& +4 \Delta_{\perp} \Delta_{z} h_{1}(n)+4 h_{2}(n)\left[\delta_{n, 2 m} \Delta_{0} \Delta_{z}+\delta_{n, 2 m+1} \Delta_{\perp}^{2}\right], \\
\beta_{\Delta_{\perp}}= & -\epsilon_{n} \Delta_{\perp}+2 \Delta_{\perp}\left(\Delta_{z}-\Delta_{0}\right) h_{2}(n)+2 \Delta_{0} \Delta_{z} h_{1}(n) \\
& +4 h_{2}(n) \Delta_{\perp}\left[\delta_{n, 2 m} \Delta_{z}+\delta_{n, 2 m+1} \Delta_{0}\right], \\
\beta_{\Delta_{z}}= & -\epsilon_{n} \Delta_{z}+2 \Delta_{z}\left(2 \Delta_{\perp}-\Delta_{0}-\Delta_{z}\right)\left[h_{1}(n)-h_{2}(n)\right] \\
& +4 \Delta_{0} \Delta_{\perp} h_{1}(n)+2 h_{2}(n) \delta_{n, 2 m}\left(\Delta_{0}^{2}+2 \Delta_{\perp}^{2}+\Delta_{z}^{2}\right),
\end{aligned}
$$

where $\delta_{n, m}$ is the Kronecker delta function, $n, m$ are integers, and $X=v, b$. Functions $h_{i}(n), i=1,2,3$, are defined as 
$h_{1}(n)=\frac{\pi(2 n-1) \csc \left(\frac{\pi}{2 n}\right)}{4 n^{2}}=1-\frac{1}{2 n}+\mathcal{O}\left(n^{-3}\right)$,

$h_{2}(n)=\frac{\pi \csc \left(\frac{\pi}{2 n}\right)}{4 n^{2}}=\frac{1}{2 n}+\mathcal{O}\left(n^{-2}\right)$,

$h_{3}(n)=\frac{\pi(n-1) \sec \left(\frac{\pi}{2 n}\right)}{4 n^{2}}=\frac{\pi}{4 n}+\mathcal{O}\left(n^{-2}\right)$.

Therefore, as $n \rightarrow \infty$ only the contribution from $h_{1}(n)$ survives, and for any finite $n, h_{2}(n)$ and $h_{3}(n)$ give rise to subleading divergences. The RG flow equations obtained by keeping only the leading divergence are shown in Eq. (11) of the main text. As we demonstrate below, at least to the leading order in $\epsilon_{n}$ expansion, inclusion of subleading divergences affects $z$ only nominally, while leaving the CLE unchanged, and we find $\nu^{-1}=\epsilon_{n}=1 / 2$.

In Sec. III A, we neglected the quantum corrections arising from the nontrivial band dispersion in the $k_{z}$ direction. Note that the quantum corrections in the RG flow equations [see Eq. (B1)] can be systematically incorporated by keeping the terms to the leading order in $1 / n$ from $h_{j}(n)$ for $j=1,2,3$ [see Eq. (B2)], following the spirit of $1 / N$ expansion, where $N$ counts the number of fermion flavors [79]. Therefore, our RG analysis is simultaneously controlled by two small parameters $\epsilon_{n}$ (measuring deviation from marginal two spatial dimensions) and $1 / n$ (controlling quantum corrections arising from band curvature along $k_{z}$ ), and only at the very end of the calculation we set $\epsilon_{n}=1 / 2$ and $n=2$. The resulting RG flow equations still support only two fixed points (similar to the ones reported in Sec. III A): (1) $\left(\Delta, \Delta_{0}, \Delta_{\perp}, \Delta_{z}\right)=(0,0,0,0)$ representing the WSMinsulator QCP in the clean system and (2) the MCP, where WSM, an insulator, and the metal meet, is now located at (obtained numerically)

$\left(\Delta, \Delta_{0}, \Delta_{\perp}, \Delta_{z}\right) \approx\left(\frac{\pi}{8 n}, \frac{1}{2}-\frac{0.40}{n}, \frac{0.195}{n}, \frac{0.185}{n}\right) \epsilon_{n}$.

The DSE at this MCP is [see Eq. (12)]

$$
z=1+\left(\frac{1}{2}-\frac{0.02}{n}\right) \epsilon_{n}
$$

which for the physical relevant situation $\epsilon_{n}=1 / 2$ and $n=2$ yields $z=1.245$, extremely close to the one reported in Sec. III A, namely, $z=1.25$, obtained by neglecting quantum corrections arising from the nontrivial dispersion in the $k_{z}$ direction. Therefore, our proposed methodology allows us to capture quantum corrections and extract the critical exponents at the MCP in a controlled fashion. The CLE, however, does not receive any $1 / n$ corrections, yielding $\nu^{-1}=\epsilon_{n}$ as before. The resulting phase diagram after accounting for $1 / n$ corrections is shown in Fig. 21(a).

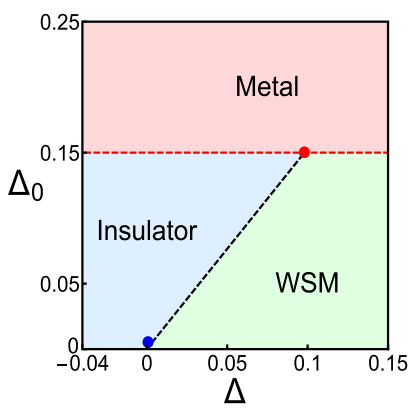

(a)

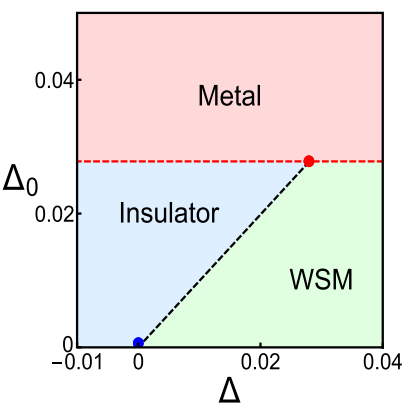

(b)
FIG. 21. The phase diagram of a dirty Weyl material residing in close proximity to WSM-insulator QPT, obtained by solving the RG flow equations Eq. (B1) for (a) and Eq. (B5) for (b). Here, $\Delta$ is the tuning parameter for WSM-insulator transition in the clean system and $\Delta_{0}$ is the strength of random charge impurities. These two phase diagrams are qualitatively similar to the one obtained numerically, see Fig. 2 (left), as the WSM-insulator phase boundary shifts toward the semimetallic side with increasing (but weak) disorder.

\section{2. $\epsilon_{d}^{\prime}$ expansion about lower critical dimension}

In this section, we demonstrate the role of disorder in the vicinity of WSM-insulator QPT perturbatively using an $\epsilon_{d}^{\prime}$ expansion near the lower critical dimension $d_{l}=5 / 2$ in the theory, see Ref. [104], where $\epsilon_{d}^{\prime}=d-5 / 2$. As we will see, the outcomes are qualitatively the same as in the $\epsilon_{n}$ regularization scheme. The exact values of the critical exponents are, however, different from the ones announced in Sec. III, although only slightly so, at least to the one-loop order. Upon integrating the fast modes within the shell $E_{c} e^{-l}<\sqrt{v^{2} k_{\perp}^{2}+b^{2} k_{z}^{4}}<E_{c}$, where $E_{c}$ is the ultraviolet energy cutoff for critical excitations residing at the WSMinsulator QCP, we arrive at the following flow equations to the leading order in $\epsilon_{d}^{\prime}$ expansion:

$$
\begin{aligned}
\beta_{X}= & -5 X\left(\Delta_{0}+2 \Delta_{\perp}+\Delta_{z}\right)=(1-z) X, \\
\beta_{\Delta}= & \Delta+(\Delta-1)\left[\Delta_{0}-2 \Delta_{\perp}+\Delta_{z}\right], \\
\beta_{\Delta_{0}}= & -\epsilon_{d}^{\prime} \Delta_{0}+10 \Delta_{0}\left(\Delta_{0}+2 \Delta_{\perp}+\Delta_{z}\right)-16 \Delta_{z} \Delta_{\perp}, \\
\beta_{\Delta_{\perp}}= & -\epsilon_{d}^{\prime} \Delta_{\perp}+2 \Delta_{\perp}\left(\Delta_{z}-\Delta_{0}\right)+4 \Delta_{z}\left(\Delta_{\perp}-2 \Delta_{0}\right), \\
\beta_{\Delta_{z}}= & -\epsilon_{d}^{\prime} \Delta_{z}+6 \Delta_{z}\left(2 \Delta_{\perp}-\Delta_{0}-\Delta_{z}\right) \\
& +4\left(\Delta_{0}^{2}+\Delta^{2}+\Delta_{0} \Delta_{z}-4 \Delta_{0} \Delta_{\perp}+2 \Delta_{\perp}^{2}\right),
\end{aligned}
$$

for $X=v, b$, after defining the dimensionless disorder coupling constant as $\Delta_{j} \alpha \rightarrow \Delta_{j}$, for $j=0, \perp$, $z$, where $\alpha=E_{c}^{\epsilon_{d}^{\prime}} /\left(20 \pi^{2} v^{2} b^{1 / 2}\right)$ and $\Delta / E_{c} \rightarrow \Delta$. Then, the $\beta$ function for $v$ and $b$ in the presence of disorder yields a scaledependent dynamic scaling exponent:

$$
z(l)=1+5\left[\Delta_{0}+2 \Delta_{\perp}+\Delta_{z}\right](l) .
$$

The coupled RG flow equations from Eq. (B5) also support only two fixed points: (i) $\left(\Delta, \Delta_{0}, \Delta_{\perp}, \Delta_{z}\right)=$ $(0,0,0,0)$, representing the WSM-insulator QCP in the 
clean limit [the blue dot in Fig. 21(b)], and (ii) $\left(\Delta, \Delta_{0}, \Delta_{\perp}, \Delta_{z}\right)=(0.058,0.056,0.01,0.02) \epsilon_{d}^{\prime}$, representing a multicritical point. The critical exponents at this multicritical point for the anisotropic critical semimetalmetal transition are

$$
\nu^{-1}=\epsilon_{d}^{\prime}, \quad z=1+0.48 \epsilon_{d}^{\prime},
$$

which is extremely close to the ones reported in Sec. III A, for $\epsilon_{d}^{\prime}=1 / 2$, leading to $z=1.24$ and $\nu=2$. Therefore, both methods produce qualitatively similar results near the WSM-insulator QPT, and the obtained critical exponents for anisotropic semimetal-metal transition are extremely close to each other, at least to the leading order. The resulting phase diagram is shown in Fig. 21(b).

\section{APPENDIX C: DETAILS OF $\epsilon_{m}$ EXPANSION}

In this appendix, we display the detailed analysis of various one-loop diagrams, shown in Fig. 9, within the framework of an $\epsilon_{m}$ expansion.

\section{Self-energy}

Let us first consider the self-energy diagram in Fig. 9(a). The expression for the self-energy reads

$$
\begin{aligned}
\Sigma(i \omega, \mathbf{k}) & =\sum_{N} \int \frac{d^{d} \mathbf{q}}{(2 \pi)^{d}} N G_{0}(i \omega, \mathbf{k}-\mathbf{q}) N \frac{\Delta_{N}}{q^{m}} \\
& \equiv \sum_{N} \Sigma_{N}(i \omega, \mathbf{k}),
\end{aligned}
$$

with $d=3$, the summation is taken over all eight types of disorder (see Table III), and $q \equiv|\mathbf{q}|$.

The contribution from the one-loop self-energy diagram from the disorder represented by the matrix $N$ reads

$$
\Sigma_{N}(i \omega, \boldsymbol{k})=-i \Delta_{N} \int \frac{d^{3} \mathbf{q}}{(2 \pi)^{3}} \frac{N\left[\gamma_{0} \omega+v \gamma_{j}(k-q)_{j}\right] N}{\left[\omega^{2}+v^{2}(\mathbf{k}-\mathbf{q})^{2}\right] q^{m}} .
$$

We will evaluate the temporal and spatial components of the self-energy diagram separately. Let us first set $\boldsymbol{k}=0$, for which

$$
\begin{aligned}
\Sigma_{N}(i \omega, 0)= & \Delta_{N}(-i \omega) \frac{N \gamma_{0} N}{v^{3-m}} \int \frac{d^{3} \mathbf{q}}{(2 \pi)^{3}} \frac{1}{\left(\omega^{2}+q^{2}\right) q^{m}} \\
= & \Delta_{N}(-i \omega) \frac{N \gamma_{0} N}{v^{3-m}} \frac{\Gamma\left(1+\frac{m}{2}\right)}{\Gamma(m / 2)} \\
& \times \int_{0}^{1} d x x^{m / 2-1} \int \frac{d^{3} \mathbf{q}}{(2 \pi)^{3}} \frac{1}{\left[q^{2}+(1-x) \omega^{2}\right]^{1+m / 2}},
\end{aligned}
$$

where $x$ is the Feynman parameter. Upon completing the integrals over $q$ and $x$, and setting $m=1-\epsilon$ (for brevity, we use here shorthand notation $\epsilon_{m} \rightarrow \epsilon$ ), we obtain

$$
\Sigma_{N}(i \omega, 0)=\left[i N \gamma_{0} \omega N\right]\left(\frac{\Delta_{N}}{2 \pi^{2} v^{2}}\right) \frac{1}{\epsilon}+\mathcal{O}(1)
$$

Next we set $\omega=0$, and the spatial component of selfenergy correction is then given by

$$
\begin{aligned}
\Sigma_{N}(0, \boldsymbol{k})= & \Delta_{N}\left[-i N \gamma_{j} N\right] \frac{1}{v^{3-m}} \int \frac{d^{3} \mathbf{q}}{(2 \pi)^{3}} \frac{(k-q)_{j}}{(\mathbf{k}-\mathbf{q})^{2} q^{m}} \\
= & \Delta_{N}\left(\frac{-i N \gamma_{j} N}{v^{3-m}}\right) \frac{\Gamma\left(1+\frac{m}{2}\right)}{\Gamma(m / 2)} \int_{0}^{1} d x x^{m / 2-1} \\
& \times \int \frac{d^{3} \mathbf{q}}{(2 \pi)^{3}} \frac{(k-q)_{j}}{\left[q^{2}-2(1-x) \mathbf{q} \cdot \mathbf{k}+(1-x) k^{2}\right]^{1+m / 2}} .
\end{aligned}
$$

After shifting the momentum variable according to $\mathbf{q}-(1-x) \mathbf{k} \rightarrow \mathbf{q}$ and setting $m=1-\epsilon$, we obtain

$$
\Sigma_{N}(0, \boldsymbol{k})=\left[i N \gamma_{j} k_{j} N\right]\left(\frac{\Delta_{N}}{2 \pi^{2} v^{2}}\right) \frac{k^{\epsilon}}{3 \epsilon}+\mathcal{O}(1) .
$$

Hence, the total self-energy correction reads

$$
\Sigma_{N}(i \omega, \boldsymbol{k})=i N\left[\gamma_{0} \omega+\frac{1}{3} \gamma_{j} k_{j}\right] N \Delta_{N} \frac{1}{\epsilon}+\mathcal{O}(1),
$$

where we have redefined $\Delta_{N} k^{\epsilon} /\left(2 \pi^{2} v^{2}\right) \rightarrow \Delta_{N}$, which is Eq. (23) in the main text.

\section{Vertex}

The vertex correction for the disorder vertex shown in Fig. 9(b) with the matrix $N$ reads

$V_{N}(\mathbf{k})=\sum_{M} \int \frac{d^{3} \mathbf{q}}{(2 \pi)^{3}} M G_{0}(0, \mathbf{k}-\mathbf{q}) N G_{0}(0, \mathbf{k}-\mathbf{q}) M \frac{\Delta_{M}}{q^{m}}$,

where we kept only one external momentum as an infrared regulator. The last expression can be compactly written as

$$
V_{N}(\mathbf{k})=-\sum_{M}\left[M \gamma_{j} N \gamma_{l} M\right] \frac{\Delta_{M}}{v^{2}} I_{j l}(\mathbf{k}),
$$

where

$$
I_{j l}(\mathbf{k})=\int \frac{d^{3} \mathbf{q}}{(2 \pi)^{3}} \frac{(k-q)_{j}(k-q)_{l}}{(\mathbf{q}-\mathbf{k})^{4} q^{m}} .
$$

We now present the evaluation of the above integral: 


$$
\begin{aligned}
I_{j l}= & \int \frac{d^{3} \mathbf{q}}{(2 \pi)^{3}} \frac{(k-q)_{j}(k-q)_{l}}{(\mathbf{k}-\mathbf{q})^{4} q^{m}} \\
= & \frac{\Gamma\left(2+\frac{m}{2}\right)}{\Gamma(m / 2)} \int_{0}^{1} d x x(1-x)^{m / 2-1} \\
& \times \int \frac{d^{3} \mathbf{q}}{(2 \pi)^{3}} \frac{(k-q)_{j}(k-q)_{l}}{\left[x(\mathbf{k}-\mathbf{q})^{2}+(1-x) q^{2}\right]^{2+m / 2}} .
\end{aligned}
$$

After shifting the momentum variable as $\mathbf{q}-x \mathbf{k} \rightarrow \mathbf{q}$, we obtain

$$
\begin{aligned}
I_{j l}= & \frac{\Gamma\left(2+\frac{m}{2}\right)}{\Gamma(m / 2)} \int_{0}^{1} d x x(1-x)^{m / 2-1} \\
& \times \int \frac{d^{3} \mathbf{q}}{(2 \pi)^{3}} \frac{[q-(1-x) k]_{j}[q-(1-x) k]_{l}}{\left[q^{2}+x(1-x) k^{2}\right]^{2+m / 2}} \\
= & -\frac{k^{\epsilon}}{2 \pi^{2}} \frac{\delta_{j l}}{3 \epsilon}+\mathcal{O}(1),
\end{aligned}
$$

after taking $m=1-\epsilon$, since only the $q$-dependent part in the numerator of the integrand yields a divergent contribution. We use the last expression to obtain Eq. (30) in the main text.

\section{Ladder crossing}

We now show computation of two ladder diagrams from Fig. 5, in the $\epsilon_{m}$-expansion scheme. After setting all the external frequencies to zero, Fig. 5(c) yields

$$
\begin{aligned}
(5 \mathrm{c})= & \Delta_{M} \Delta_{N} \int \frac{d^{3} \mathbf{q}}{(2 \pi)^{3}}\left[M \frac{i \gamma_{l}\left(p_{1}-q\right)_{l}}{\left(\mathbf{p}_{1}-\mathbf{q}\right)^{2}} N\right] \\
& \times\left[N \frac{i \gamma_{s}\left(p_{2}+q\right)_{s}}{\left(\mathbf{p}_{2}+\mathbf{q}\right)^{2}} M\right] \frac{1}{|\mathbf{q}|^{m}\left|\mathbf{p}_{3}-\mathbf{p}_{1}-\mathbf{q}\right|^{m}} .
\end{aligned}
$$

Taking then $p_{1}=p_{3}=0$ and keeping only the most singular contribution, we obtain

$$
(5 \mathrm{c}) \sim \Delta_{M} \Delta_{N} \int \frac{d^{3} \mathbf{q}}{(2 \pi)^{3}} \frac{1}{|\mathbf{q}|^{2 m}\left(\mathbf{p}_{2}+\mathbf{q}\right)^{2}} .
$$

Here, we used that $\left[\gamma_{l}, X\right]=0$ or $\left\{\gamma_{l}, X\right\}=0$ for all $j=1$, 2,3 and $X=M, N$, as well as $[M, N]=0$ or $\{M, N\}=0$. Computation of the last integral yields

$$
(5 \mathrm{c}) \sim \Delta_{M} \Delta_{N}\left(\mathbf{p}_{2}^{2}\right)^{1 / 2-m} \frac{\Gamma\left(\frac{1}{2}\right) \Gamma\left(\frac{3}{2}-m\right) \Gamma\left(m-\frac{1}{2}\right)}{(4 \pi)^{3 / 2} \Gamma(m) \Gamma(2-m)},
$$

which is finite in the expansion in $\epsilon_{m}=1-m$ as $m \rightarrow 0$. This is also expected based on the power counting of the integral in Eq. (C14). Figure 5(d) reads

$$
\begin{aligned}
(5 \mathrm{~d})= & \Delta_{M} \Delta_{N} \int \frac{d^{3} \mathbf{q}}{(2 \pi)^{3}}\left[M \frac{i \gamma_{l}\left(p_{1}-q\right)_{l}}{\left(\mathbf{p}_{1}-\mathbf{q}\right)^{2}} N\right] \\
& \times\left[M \frac{i \gamma_{s}\left(p_{1}+p_{2}-p_{3}-q\right)_{s}}{\left(\mathbf{p}_{1}+\mathbf{p}_{2}-\mathbf{p}_{3}-\mathbf{q}\right)^{2}} N\right] \\
& \times \frac{1}{|\mathbf{q}|^{m}\left|\mathbf{p}_{1}-\mathbf{p}_{2}-\mathbf{q}\right|^{m}} .
\end{aligned}
$$

Taking then $\mathbf{p}_{1}=\mathbf{p}_{3}=0$ and keeping only the most singular contribution, we obtain

$$
(5 \mathrm{~d}) \sim \Delta_{M} \Delta_{N} \int \frac{d^{3} \mathbf{q}}{(2 \pi)^{3}} \frac{1}{|\mathbf{q}|^{2 m}\left(\mathbf{p}_{2}-\mathbf{q}\right)^{2}},
$$

identical to the integral from Eq. (C14), after substituting $\mathbf{q} \rightarrow-\mathbf{q}$. Therefore this diagram is also ultraviolet finite, confirming that both ladder diagrams are finite in the $\epsilon_{m}$ expansion, irrespective of the choice of $M$ and $N$.

The reason for these two diagrams yielding ultraviolet finite contribution is the following: since the disorder propagator is momentum dependent in the $\epsilon_{m}$-expansion scheme (unlike the situation in $\epsilon_{d}$ expansion), only the selfenergy and vertex diagrams [see Fig. 9] containing only one disorder line are ultraviolet divergent and contribute RG flow equations. By contrast, each of the two ladder diagrams [see Figs. 5(c) and 5(d)] contains two disorder lines, yielding UV finite contribution and thus do not influence the RG flow equations. Now readers can convince themselves that such a distinction between these two sets of Feynman diagrams persists to any order in perturbation theory. Hence, in the $\epsilon_{m}$-expansion scheme, ladder diagrams never contribute and we do not generate any short-range disorder.

\section{APPENDIX D: LATTICE REALIZATION OF GENERIC DISORDER IN WEYL SEMIMETAL}

In this appendix, we demonstrate the lattice realization of 16 possible fermionic bilinears (shown in Table III) from the two-band tight-binding model, displayed in Eqs. (5) and (3). By virtue of the chosen tight-binding model, our construction is based on two features

(1) Since two Weyl nodes are located on the $k_{z}$ axis at $\pm k_{z}^{0}= \pm \pi /(2 a)$, any fermionic bilinear odd under the exchange of two Weyl nodes can be realized by adding $h=\sum_{\mathbf{k}} \Psi_{\mathbf{k}}^{\dagger} \sin \left(k_{z} a\right) \sigma_{j} \Psi_{\mathbf{k}}$ to the tight-binding model, where $j=0,1,2,3$. Such perturbation corresponds to an imaginary hopping along the $z$ direction, and does not renormalize the bandwidth.

(2) Any fermionic bilinear that couples two Weyl nodes, which therefore necessarily breaks translational symmetry, can be realized through a periodic and commensurate modulation of the nearest-neighbor hopping amplitude, but only along the $z$ direction. 

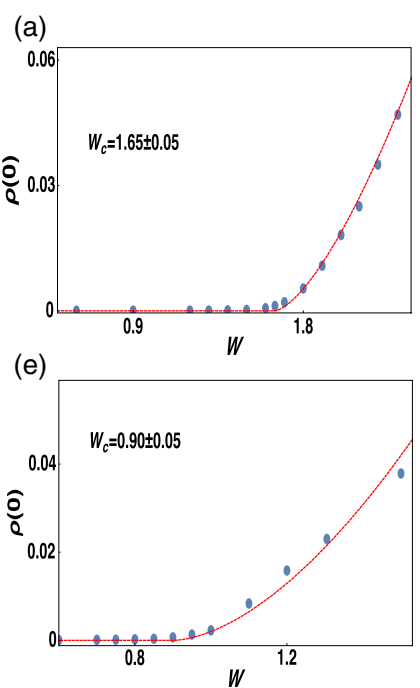

(b)

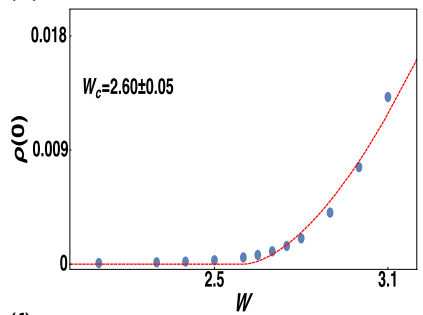

(f)

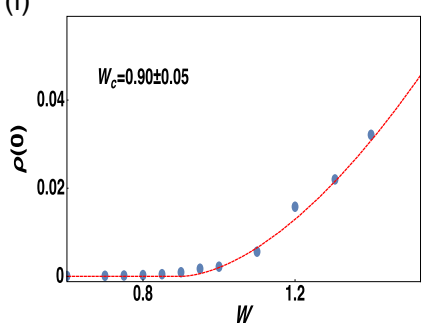

(c)

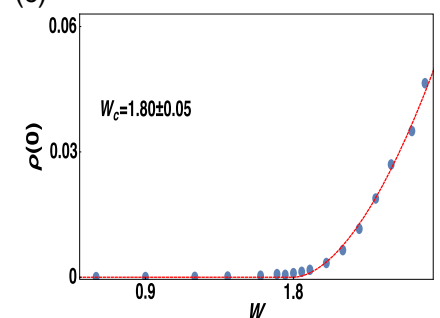

(g)

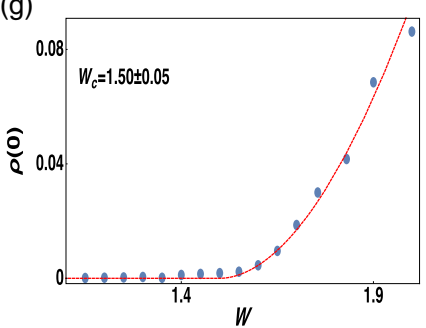

(d)

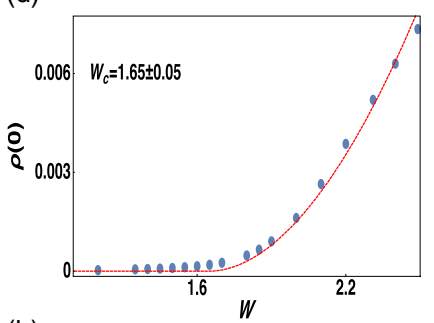

(h)

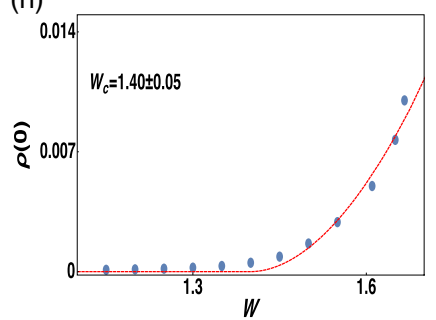

FIG. 22. Scaling of the ADOS at zero energy $[\varrho(0)]$ as a function of disorder strength $W$ (chosen to be uniformly and independently distributed within a box $[-W, W]$ ) for (a) potential, (b) axial, (c) axial current (or magnetic), (d) current, (e) temporal tensor (or spinorbit), (f) spatial tensor (or axial magnetic), (g) scalar mass, and (h) pseudoscalar mass disorder. The results are obtained by using KPM in a cubic lattice with linear dimension $L=220$ in each direction. Note $\varrho(0)$ is pinned to zero up to a critical strength of disorder $W_{c}$, quoted in each panel (see also Tables I and II), and then it becomes finite, indicating the onset of a metallic phase. We reduce the uncertainty in determining the location of $W_{c}$ within the error bar \pm 0.05 , allowing us to minimize the fitting error in the determination of $z$ and $\nu$ (see Appendix E and Table IV).

With these two construction principles we can realize all 16 fermion bilinears by adding the following terms to the tight-binding Hamiltonian:

(1) regular chemical potential,

$$
\sum_{\mathbf{r}} \Psi_{\mathbf{r}}^{\dagger} V(\mathbf{r}) \sigma_{0} \Psi_{\mathbf{r}}
$$

(2) axial chemical potential,

$$
\sum_{\mathbf{r}} \Psi_{\mathbf{r}}^{\dagger}\left[\frac{i V(\mathbf{r})}{2} \sigma_{0}\right] \Psi_{\mathbf{r}+\hat{e}_{3}}+\text { H.c. }
$$

(3) Abelian current,

$$
\begin{aligned}
\sum_{\mathbf{r}} & \left\{\Psi_{\mathbf{r}}^{\dagger}\left[\frac{i V(\mathbf{r})}{2} \sigma_{3}\right] \Psi_{\mathbf{r}+\hat{e}_{3}}+\right.\text { H.c. } \\
& \left.+\Psi_{\mathbf{r}}^{\dagger} V(\mathbf{r})\left(\sigma_{1}+\sigma_{2}\right) \Psi_{\mathbf{r}}\right\},
\end{aligned}
$$

(4) Abelian axial current,

$$
\begin{aligned}
\sum_{\mathbf{r}}\left\{\Psi_{\mathbf{r}}^{\dagger}\left[\frac{i V(\mathbf{r})}{2}\left(\sigma_{1}+\sigma_{2}\right)\right] \Psi_{\mathbf{r}+\hat{e}_{3}}\right. \\
\left.+ \text { H.c. }+\Psi_{\mathbf{r}}^{\dagger} V(\mathbf{r}) \sigma_{3} \Psi_{\mathbf{r}}\right\},
\end{aligned}
$$

(5) temporal components of tensor,

$$
\sum_{\mathbf{r}} \sum_{j}(-1)^{j} \Psi_{\mathbf{r}, j}^{\dagger} V(r)\left[\sigma_{1}+\sigma_{2}+i \sigma_{0}\right] \Psi_{\mathbf{r}, \hat{e}_{3}, j+1}+\text { H.c. }
$$

(6) spatial components of tensor,

$$
\sum_{\mathbf{r}} \sum_{j}(-1)^{j} \Psi_{\mathbf{r}, j}^{\dagger} V(r)\left[\sigma_{0}+i \sigma_{1}+i \sigma_{2}\right] \Psi_{\mathbf{r}, \hat{e}_{3}, j+1}+\text { H.c., }
$$

(7) scalar mass,

$$
\sum_{\mathbf{r}} \sum_{j}(-1)^{j} \Psi_{\mathbf{r}, j}^{\dagger}\left[V(r) \sigma_{3}\right] \Psi_{\mathbf{r}, \hat{e}_{3}, j+1}+\text { H.c. },
$$

(8) pseudoscalar mass,

$$
\sum_{\mathbf{r}} \sum_{j}(-1)^{j} \Psi_{\mathbf{r}, j}^{\dagger}\left[i V(r) \sigma_{3}\right] \Psi_{\mathbf{r}, \hat{e}_{3}, j+1}+\text { H.c. }
$$

Thus, within the simplest realization of a Weyl semimetal from a tight-binding model on a cubic lattice, one can realize all possible disorder couplings by choosing $V(\mathbf{r})$ as a random variable, and numerically study possible WSMmetal QPTs. The scaling of ADOS at zero energy for all the above eight disorders is shown in Fig. 22. 


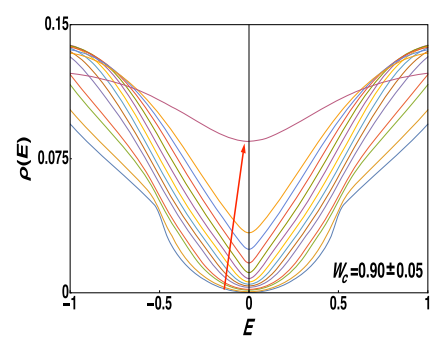

(a)

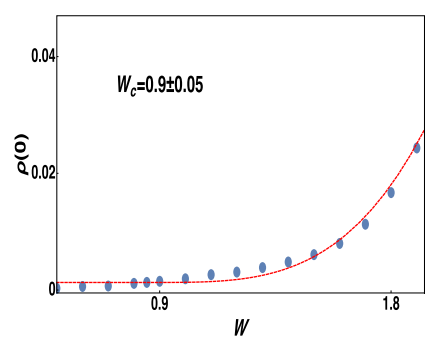

(b)

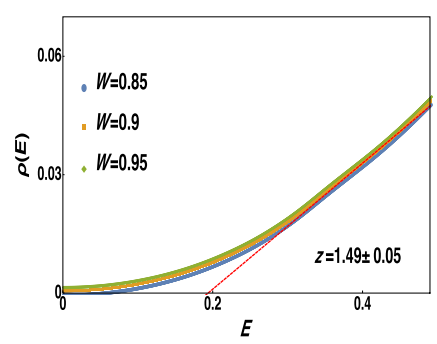

(c)

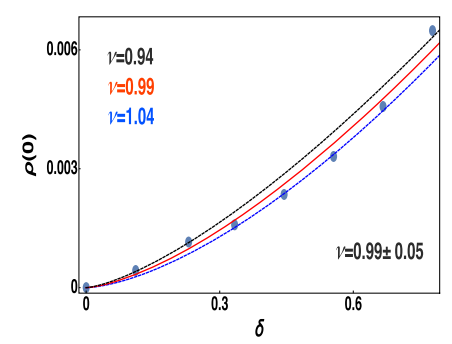

(d)

FIG. 23. Numerical analysis of average DOS in the presence of correlated potential disorder [see Appendix F and Eq. (F1)]. Scaling of (a) average DOS $[\varrho(E)]$ with increasing strength of disorder (in the direction of the red arrow), (b) average DOS at zero energy $[\varrho(0)]$ with increasing disorder in the system, yielding critical disorder strength $W_{c}=0.90 \pm 0.05$, (c) average DOS $[Q(E)]$ around critical disorder $W=0.85$ (blue), 0.90 (yellow), and 0.95 (green) [fitting $\varrho(E)$ with $|E|^{d / z-1}$, we obtain $z=1.49 \pm 0.05$ ], (d) $\varrho(0)$ with the reduced distance from the WSM-metal critical point $(\delta)$, yielding $\nu=0.99 \pm 0.05$ with $W_{c}=0.90$ and $z=1.49$. For discussion on error analysis in the determination of $\nu$, see Appendixes E and F, as well as Table IV (the second to the last row).

\section{APPENDIX E: DETAILS OF DATA ANALYSIS}

In this appendix, we present quintessential details of data analysis, which we employ for (a) anisotropic semimetalmetal QPT through the MCP [the blue dot in Fig. 2 (left)] as well as (b) WSM-metal QPT [for both uncorrelated and correlated disorder (see Appendix F)].

\section{Estimation of $W_{c}$}

We determine the critical strength of disorder $\left(W_{c}\right)$ by computing the average DOS at zero energy $\varrho(0)$. Note $\varrho(0)=0$ in the semimetallic phase as well as at the semimetal-metal QCP. But $\varrho(0)$ is finite in a metal. Hence, by computing $Q(0)$ we can pin down $W_{c}$, as shown in Figs. 7(a), 22, and 23(b). We minimize the error $\delta W_{c}$ in determining $W_{c}$ by increasing the number of data points around $W_{c}$, and throughout, $\delta W_{c}=0.05$. Note small $\delta W_{c}$ is the source of small "fitting error" in the quoted values of $z$ and $\nu$.

\section{Estimation of $z$}

To determine the DSE $z$, we compare $Q(E)$ versus $|E|^{d / z-1}$, for $W_{c}-\delta W_{c}, W_{c}$, and $W_{c}+\delta W_{c}$. Since continuous semimetal-metal QPT is always characterized by a unique $z$, we fit $Q(E)$ for a specific value of $z$. But, due to the finite-size effects (which are nonuniversal and also depend on the choice of disorder distribution), such a fit never goes through zero at $E=0$, although $\varrho(0) \approx 0$ (within numerical accuracy). Hence, to find $z$ we search for its value that yields good fit with $Q(E)$ at finite energy [i.e., we target to fit $\varrho(E)$ with $|E|^{d / z-1}$ within the quantumcritical regime, where finite-size effects are nominal]. For three values of $W$, namely, $W_{c}-\delta W_{c}, W_{c}$, and $W_{c}+\delta W_{c}$, we obtain three values of $z$, namely, $z-\delta z, z$, and $z+\delta z$, where $\delta z$ is the fitting error associated with $z$ [see Tables I and II and Figs. 7(b) and 23(c)]. The red lines shown in Figs. 7(b), 13 (first column), 14 (first column), and 23(c) represent plots of $Q(E)$ versus $|E|^{d / z-1}$ for the mean value of $z$.

\section{Estimation of $\nu$}

Finally, we determine CLE $\nu$, for which we compare $\varrho(0)$ in the metallic phase with $\delta=\left(W-W_{c}\right) / W_{c}$ (where $W_{c}$ is the mean value of critical disorder strength), since $\varrho(0) \sim \delta^{(d-z) \nu}$. Within the maximally allowed range of disorder $W_{c}<W<W_{*}$ (due to the inevitable presence of a subsequent Anderson transition, explained below), we fit $\varrho(0)$ versus $\delta^{(d-z) \nu}$, yielding CLE $\nu \pm \delta \nu$ with the mean value of DSE $z$, reported in Fig. 7(c), the second column of Figs. 13 and 14, and in Fig. 23(d). The black, red, and blue curves are, respectively, shown for $\nu \in\{\nu-\delta \nu, \nu, \nu+\delta \nu\}$, encompassing all data points.

To further improve our numerical analysis of $\nu$, we generate two additional data sets for $\varrho(0)$ versus $\delta$, with $W_{c} \rightarrow$ $W_{c} \pm \delta W_{c}$, but still within the range $W_{c} \pm \delta W_{c}<$ $W<W_{*}$. Performing the same analysis explained above, we obtain another range of CLE, $\nu$. Finally, for all three sets of $\varrho(0)$ versus $\delta$, we extract the CLE taking $z \rightarrow z \pm \delta z$. With such extensive data analysis, summarized in Table IV, we acquire maximal fitting error in the determination of $\nu$, and these values are quoted in Tables I and II (for WSM-metal QPT), reported in Sec. III B for anisotropic semimetal-metal QPT through the MCP and in Appendix F for correlated potential disorder.

Finally, we highlight an important issue related to the range of disorder $(\delta)$ over which we perform numerical analysis for $\nu$. Note that a three-dimensional Weyl metal undergoes a second QPT into the AI phase (discussed in Sec. IX; see also Fig. 1). Across the Anderson transition, although average DOS at zero energy $\left[\varrho_{a}(0)\right]$ remains smooth, it decreases monotonically. As shown in Fig. 18(a), Anderson transition (for potential disorder) takes place at $W_{c, 2} \approx 9.30$, but $\varrho_{a}(0)$ starts to decrease for much weaker disorder $W \geq 2.90$. On the other hand, the 
TABLE IV. Details of the data analysis for the computation of the correlation length exponent $\nu$ across the WSM-metal QPT driven by potential $\left(\Delta_{V}\right)$, axial potential $\left(\Delta_{A}\right)$, magnetic $\left(\Delta_{M}\right)$, current $\left(\Delta_{C}\right)$, spin-orbit $\left(\Delta_{S O}\right)$, axial magnetic $\left(\Delta_{A M}\right)$, scalar mass $\left(\Delta_{S}\right)$, and pseudoscalar mass $\left(\Delta_{P S}\right)$ disorder (see Table III for definition), where the disorder is assumed to be uniformly and independently distributed within $[-W, W]$ (first eight rows). We here show the variation of $\nu$ with (a) the variation of the location of the WSM-metal QCP (denoted by $W_{c} \pm \delta W_{c}$ ) and (b) from the fitting error of $z$ (denoted by $z \pm \delta z$ ). See Tables I and II for $W_{c}, z$, and $\delta z$, and throughout we have $\delta W_{c}=0.05$. The second to the last row represents the same analysis but in the presence of correlated potential disorder $\left(\Delta_{V}^{\text {corr }}\right)$, discussed in Appendix F, while the last row shows similar analysis across the potential disorder-driven critical anisotropic semimetalmetal transition through the multicritical point (discussed in Sec. III). The quantities in parentheses represent corresponding fitting error for a given value of $\nu$ for the specific value of critical disorder strength and dynamic scaling exponent; see Appendix E for discussion. Each value of $\nu$ and the corresponding fitting error is determined by comparing $Q(0)$ with $\delta^{(d-z) \nu}$ for given values of $W_{c}$ and $z$, see, for example, Fig. 7(c), second column of Figs. 13 and 14, and Fig. 23(d). The last column shows the corresponding values of the correlation length exponent, accompanied by maximal fitting error. These analyses were performed in the largest system (see Secs. III B and VI and Appendix F for details). Data collapse in Figs. 8, 13, 14, and 24 are shown with the values for $W_{c}, z$, and $\nu$ shown in bold font.

\begin{tabular}{|c|c|c|c|c|c|c|c|c|c|c|}
\hline \multirow[b]{3}{*}{ Disorder } & \multicolumn{3}{|c|}{$W_{c}-\delta W_{c}$} & \multicolumn{3}{|c|}{$W_{c}$} & \multicolumn{3}{|c|}{$W_{c}+\delta W_{c}$} & \multirow[b]{3}{*}{$\nu$} \\
\hline & $z-\delta z$ & $z$ & $z+\delta z$ & $z-\delta z$ & $\mathbf{z}$ & $z+\delta z$ & $z-\delta z$ & $z$ & $z+\delta z$ & \\
\hline & $\nu$ & $\nu$ & $\nu$ & $\nu$ & $\nu$ & $\nu$ & $\nu$ & $\nu$ & $\nu$ & \\
\hline$\Delta_{V}$ & $0.97(0.06)$ & $1.00(0.05)$ & $1.02(0.06)$ & $0.97(0.05)$ & $\mathbf{0 . 9 9}(0.05)$ & $1.01(0.07)$ & $0.98(0.06)$ & $0.99(0.07)$ & $1.01(0.05)$ & $1.00(0.08)$ \\
\hline$\Delta_{A}$ & $1.04(0.06)$ & $1.07(0.05)$ & $1.09(0.05)$ & $1.01(0.05)$ & $\mathbf{1 . 0 3}(0.05)$ & $1.08(0.08)$ & $1.04(0.05)$ & $1.06(0.07)$ & $1.08(0.08)$ & $1.06(0.10)$ \\
\hline$\Delta_{M}$ & $0.99(0.06)$ & $1.02(0.05)$ & $1.05(0.05)$ & $1.00(0.06)$ & $\mathbf{1 . 0 3}(0.05)$ & $1.05(0.05)$ & $1.01(0.05)$ & $1.05(0.05)$ & $1.07(0.06)$ & $1.03(0.10)$ \\
\hline$\Delta_{C}$ & $1.01(0.05)$ & $1.04(0.06)$ & $1.07(0.06)$ & $0.99(0.05)$ & $\mathbf{1 . 0 3}(0.05)$ & $1.06(0.06)$ & $0.99(0.05)$ & $1.03(0.06)$ & $1.06(0.05)$ & $1.02(0.09)$ \\
\hline$\Delta_{S O}$ & $1.01(0.05)$ & $1.03(0.06)$ & $1.06(0.05)$ & $0.97(0.07)$ & 1.01 $(0.05)$ & $1.04(0.05)$ & $0.99(0.06)$ & $1.02(0.06)$ & $1.05(0.05)$ & $1.01(0.10)$ \\
\hline$\Delta_{A M}$ & $0.99(0.07)$ & $1.03(0.06)$ & $1.07(0.05)$ & $0.97(0.08)$ & 1.01 $(0.05)$ & $1.05(0.06)$ & $0.97(0.06)$ & $1.02(0.05)$ & $1.06(0.06)$ & $0.99(0.12)$ \\
\hline$\Delta_{S}$ & $0.95(0.06)$ & $0.99(0.05)$ & $1.03(0.07)$ & $0.95(0.05)$ & $\mathbf{0 . 9 7}(0.05)$ & $1.01(0.07)$ & $0.95(0.07)$ & $0.99(0.05)$ & $1.02(0.06)$ & $0.99(0.12)$ \\
\hline$\Delta_{P S}$ & $0.99(0.08)$ & $1.02(0.05)$ & $1.05(0.06)$ & $0.99(0.05)$ & $\mathbf{1 . 0 2}(0.06)$ & $1.06(0.05)$ & $0.97(0.05)$ & $1.01(0.05)$ & $1.05(0.06)$ & $1.01(0.11)$ \\
\hline$\Delta_{V}^{\text {corr }}$ & $0.96(0.07)$ & $1.00(0.05)$ & $1.04(0.06)$ & $0.96(0.05)$ & $\mathbf{0 . 9 9}(0.05)$ & $1.03(0.07)$ & $0.96(0.08)$ & $1.01(0.05)$ & $1.05(0.05)$ & $0.99(0.11)$ \\
\hline$\Delta_{V}(\mathrm{MCP})$ & $1.94(0.07)$ & $1.97(0.06)$ & $2.03(0.05)$ & $1.92(0.05)$ & $1.97(0.05)$ & $2.01(0.06)$ & $1.97(0.06)$ & $1.99(0.05)$ & $2.02(0.06)$ & $1.98(0.10)$ \\
\hline
\end{tabular}

WSM-metal QPT takes place around $W_{c, 1} \approx 1.65$. Hence, for $W>2.9$, the Weyl metal starts to approach the Anderson fixed point, and to properly extract $\nu$ associated with the WSM-metal QPT we can only fit $\varrho_{a}(0)$ with $\delta^{(d-z) \nu}$ within the range $1.65 \pm 0.05<W<2.90$, the maximally allowed range of disorder, mentioned earlier, with $W_{*}<2.90$. The maximal value of $\delta$ shown in the second column of the first row in Fig. 13 is $\approx 0.60$, yielding corresponding $W \approx 2.65$ with $W_{c}=1.65$ (ensuring that the system is still sufficiently far from the Anderson fixed point). Therefore, in our data analysis for $\nu$, we cover the maximally allowed range of disorder so that the system still falls outside the basin of attraction for the Anderson transition. No further variation of range of $\delta$ is permitted due to the very nature of the global phase diagram, shown in Fig. 1. This way we acquire a maximal fitting error in determination of $\nu$ arising from the range of $\delta$ we consider, that nonetheless encompasses all data points. We follow the same strategy for the analysis of $\nu$ in the presence of arbitrary disorder driving WSM-metal QPT or the transition across the MCP.

\section{APPENDIX F: CORRELATED DISORDER}

So far we have assumed disorder to be a random variable within the range $[-W, W]$ at each site of the cubic lattice with linear dimension $L$ in each direction. Hence, disorder is uncorrelated, which involves both intravalley as well as intervalley scattering (since in any lattice model left and right chiral Weyl points are always connected at high energies). However, our proposed scenario of the emergent superuniversality (see Sec. VIID) suggests that in the presence of generic disorder, the WSM-metal QPT is characterized by a unique set of exponents, namely, $z=$ 1.5 and $\nu=1$ (obtained from leading-order $\epsilon$ expansions, in good agreement with numerical findings). Otherwise, such emergent superuniversality does not depend on the actual nature of the disorder (see Figs. 13 and 14 and Tables I and II) nor does it depend on the distribution of disorder. To anchor the last statement we now present the numerical analysis of average DOS in the presence of correlated random charge impurities for which intervalley scattering is suppressed (although finite) by construction (at least when disorder is sufficiently weak). As we demonstrate, the universality class of the WSM-metal transition remains unaffected (within numerical accuracy) by the choice of disorder distribution, apart from causing a nonuniversal shift of WSM-metal QCP $\left(W_{c}\right)$.

We introduce a Gaussian disorder potential $W(\mathbf{r})$, such that the mean $\langle W(\mathbf{r})\rangle=0$, but

$$
\left\langle W(\mathbf{r}) W\left(\mathbf{r}^{\prime}\right)=\frac{W}{\xi^{2}} \exp \left(-\frac{\left|\mathbf{r}-\mathbf{r}^{\prime}\right|^{2}}{2 \xi^{2}}\right) .\right.
$$


In the lattice implementation, we set $\xi=4 a$, where $a$ is the lattice constant, leading to a strong suppression of intervalley scattering by a factor $\exp \left[-(\Delta k)^{2} \xi^{2} / 2\right]<10^{-34}$, where $\Delta k=\pi / a$ is the separation between two Weyl nodes [105]. Now we proceed with the numerical analysis of the average DOS using KPM in a cubic lattice with linear dimension $L=160$ in each direction. We average over 20 random disorder realizations, compute 4096 Chebyshev moments, and take trace over 12 random vectors to compute $\varrho(E)$.

First, notice that scaling of average DOS $Q(E)$ as a function of increasing disorder [see Fig. 23(a)] is similar to the ones found with box distribution (see Fig. 3), showing a smooth crossover from $\varrho(E) \sim|E|^{2}$ to $|E|$ scaling as we approach the critical disorder strength $W_{c}=0.90 \pm 0.05$ from the semimetallic side, beyond which $\varrho(0)$ becomes finite [see Fig. 23(b)] and the system enters a metallic phase. By fitting DOS $\varrho(E)$ with $|E|^{d / z-1}$, we obtain $z=1.49 \pm 0.05$; see Fig. 23(c). Finally, we compare the DOS at zero energy $\varrho(0)$ with $\delta=\left(W-W_{c}\right) / W_{c}$, and with mean values of $W_{c}(=0.90)$ and $z(=1.49)$, and find $\nu=0.99 \pm 0.05$ [see Fig. 23(d)]. We here compare $\varrho(0)$ versus $\delta$ up to $W=1.75$, such that $Q(0)$ increases smoothly within $0.90<W<1.75$ (see last paragraph of Appendix E). Finally, performing a similar data analysis by accounting for the variation of $W_{c}$ and $z$, we find $\nu=0.99 \pm 0.11$, containing maximal fitting error in the determination of $\nu$ (see second to the last row of Table IV).

Now, with the mean values of these parameters, namely, $W_{c}=0.90, z=1.49$, and $\nu=0.99$, we proceed to the data collapse. The results are displayed in Figs. 24(a) and 24(b), discerning satisfactory data collapse over a large parameter space. In corroboration with the numerical results we presented for all possible disorder with uncorrelated box distribution, the present numerical analysis for correlated potential disorder supports the following fact: the universality class of the WSM-metal QPT is insensitive to the nature of disorder as well as its distribution, which in conjunction with our field-theoretic predictions supports

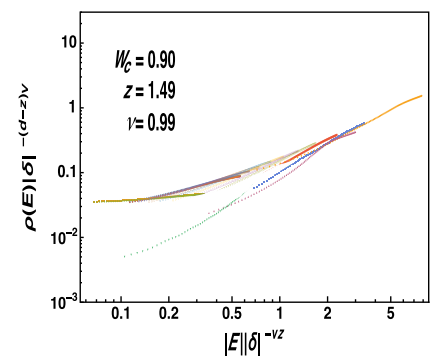

(a)

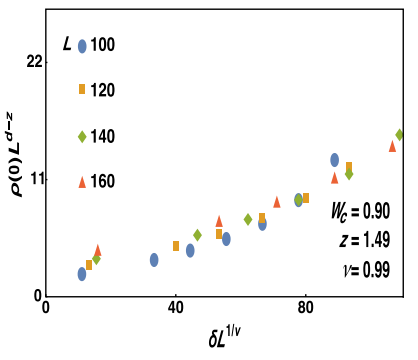

(b)
FIG. 24. Two types of data collapse in the presence of correlated potential disorder. (a) Finite energy collapse of $\varrho(E)|\delta|^{(d-z) \nu}$ versus $|E| \delta^{-\nu z}$ in a system with $L=160$. All data fall on two branches: top one corresponds to the metallic phase, while the bottom one corresponds to the semimetallic phase. (b) Finite-size data collapse of $\varrho(0) L^{d-z}$ versus $\delta L^{1 / \nu}$. For these two data collapses we take $W_{c}=0.9, z=1.49$, and $\nu=0.99$.

the proposed scenario of emergent superuniversality across the WSM-metal QPT.

\section{APPENDIX G: RG ANALYSIS IN THE PRESENCE OF GENERIC DISORDER COUPLINGS}

In this appendix, we present the coupled RG flow equations for eight disorder couplings shown in Table III, obtained within the framework of $\epsilon_{m}$ expansion (defined in Sec. IV B) and $\epsilon_{d}$ expansion (defined in Sec. IV C). We show that under generic circumstances the line of QCPs, defined in Eq. (36) (obtained from $\epsilon_{m}$ expansion) or Eq. (39) (obtained from $\epsilon_{d}$ expansion), in the $\left(\Delta_{V}, \Delta_{A}\right)$ plane (two chiral-symmetric disorders), is the legitimate solution, which provides a strong justification for the chiral superuniversality across generic disorder-driven WSM-metal QPT, qualitatively discussed in Sec. VII D.

\section{RG flow equations from $\epsilon_{m}$ expansion}

The leading-order coupled RG flow equations in the presence of all eight disorder couplings within the framework of an $\epsilon_{m}$ expansion read as

$$
\begin{gathered}
\beta_{\Delta_{V}}=\Delta_{V}\left[-\epsilon_{m}+\frac{4}{3}\left(2 \Delta_{A}+5 \Delta_{A M}+4 \Delta_{C}+4 \Delta_{M}+\Delta_{P S}+\Delta_{S}+5 \Delta_{S O}+2 \Delta_{V}\right)\right] \\
\beta_{\Delta_{A}}=\Delta_{A}\left[-\epsilon_{m}+\frac{8}{3}\left(\Delta_{A}-2 \Delta_{\mathrm{AM}}+2 \Delta_{c}+2 \Delta_{M}-\Delta_{\mathrm{PS}}-\Delta_{S}-2 \Delta_{\mathrm{SO}}+\Delta_{V}\right)\right] \\
\beta_{\Delta_{M}}=\Delta_{M}\left[-\epsilon_{m}+\frac{4}{3}\left(\Delta_{A M}-\Delta_{P S}-\Delta_{S}+\Delta_{S O}\right)\right] \\
\beta_{\Delta_{C}}=-\epsilon_{m} \Delta_{C} \\
\beta_{\Delta_{S O}}=\Delta_{S O}\left[-\epsilon_{m}+\frac{4}{3}\left(\Delta_{A M}-\Delta_{M}-\Delta_{S}+\Delta_{V}\right)\right]
\end{gathered}
$$




$$
\begin{gathered}
\beta_{\Delta_{A M}}=\Delta_{\mathrm{AM}}\left[-\epsilon_{m}-\frac{4}{3}\left(\Delta_{M}+\Delta_{P S}-\Delta_{S O}-\Delta_{V}\right)\right] \\
\beta_{\Delta_{S}}=\Delta_{S}\left[-\epsilon_{m}+\frac{4}{3}\left(2 \Delta_{A}-4 \Delta_{A M}+4 \Delta_{C}-5 \Delta_{M}+\Delta_{P S}-2 \Delta_{S}+5 \Delta_{S O}-\Delta_{V}\right)\right] \\
\beta_{\Delta_{P S}}=\Delta_{P S}\left[-\epsilon_{m}+\frac{4}{3}\left(2 \Delta_{A}+5 \Delta_{A M}+4 \Delta_{C}-5 \Delta_{M}-2 \Delta_{P S}+\Delta_{S}-4 \Delta_{S O}-\Delta_{V}\right)\right]
\end{gathered}
$$

The above set of coupled flow equations only supports a line of QCPs, given by Eq. (36). Along the entire line of QCPs the exponents are given by $\nu^{-1}=\epsilon_{m}+\mathcal{O}\left(\epsilon_{m}^{2}\right)$ and $z=1+\epsilon_{m} / 2+\mathcal{O}\left(\epsilon_{m}^{2}\right)$ in three dimensions, to the leading order in $\epsilon_{m}$. Therefore, for Gaussian white noise distribution $\left(\epsilon_{m}=1\right)$, we obtain $\nu=1$ and $z=3 / 2$. This outcome strongly supports the proposed emergent superuniversality across the WSM-metal QPT, driven by arbitrary disorder.

\section{RG flow equations from $\epsilon_{d}$ expansion}

The coupled RG flow equations for eight symmetry-allowed disorder couplings to the leading order in the $\epsilon_{d}$ expansion read as

$$
\begin{gathered}
\beta_{\Delta_{V}}=-\epsilon_{d} \Delta_{V}+2 \Delta_{V}\left(\Delta_{A}+3 \Delta_{A M}+3 \Delta_{C}+3 \Delta_{M}+\Delta_{P S}+\Delta_{S}+3 \Delta_{S O}+\Delta_{V}\right)+4\left(2 \Delta_{C} \Delta_{M}+\Delta_{A M} \Delta_{P S}+\Delta_{S} \Delta_{S O}\right), \\
\beta_{\Delta_{A}}=-\epsilon_{d} \Delta_{A}+2 \Delta_{A}\left(\Delta_{A}-3 \Delta_{A M}+3 \Delta_{C}+3 \Delta_{M}-\Delta_{P S}-\Delta_{S}-3 \Delta_{S O}+\Delta_{V}\right)+4\left(\Delta_{A M}^{2}+\Delta_{C}^{2}+\Delta_{M}^{2}+\Delta_{S O}^{2}\right), \\
\beta_{\Delta_{M}}=-\epsilon_{d} \Delta_{M}+\frac{2}{3} \Delta_{M}\left(-\Delta_{A}+\Delta_{A M}+\Delta_{C}+\Delta_{M}-\Delta_{P S}-\Delta_{S}+\Delta_{S O}-\Delta_{V}\right)+\frac{4}{3}\left(2 \Delta_{A} \Delta_{M}+7 \Delta_{A M} \Delta_{S O}+2 \Delta_{C} \Delta_{V}+\Delta_{P S} \Delta_{S}\right),
\end{gathered}
$$

$\beta_{\Delta_{C}}=-\Delta_{C}+\frac{2}{3} \Delta_{C}\left(-\Delta_{A}-\Delta_{A M}+\Delta_{C}+\Delta_{M}+\Delta_{P S}+\Delta_{S}-\Delta_{S O}-\Delta_{V}\right)+\frac{8}{3}\left(\Delta_{A} \Delta_{C}+\Delta_{A M} \Delta_{S}+\Delta_{M} \Delta_{V}+\Delta_{P S} \Delta_{S O}\right)$,

$$
\beta_{\Delta_{S O}}=\epsilon_{d} \Delta_{S O}-\frac{2}{3} \Delta_{S O}\left(\Delta_{A}-\Delta_{A M}-\Delta_{C}+\Delta_{M}-\Delta_{P S}+\Delta_{S}+\Delta_{S O}-\Delta_{V}\right)+\frac{4}{3}\left(2 \Delta_{A} \Delta_{S O}+7 \Delta_{A M} \Delta_{M}+2 \Delta_{C} \Delta_{P S}+\Delta_{S} \Delta_{V}\right),
$$

$\beta_{\Delta_{A M}}=-\epsilon_{d} \Delta_{A M}-\frac{2}{3} \Delta_{A M}\left(\Delta_{A}+\Delta_{A M}-\Delta_{C}+\Delta_{M}+\Delta_{P S}-\Delta_{S}-\Delta_{S O}-\Delta_{V}\right)+\frac{4}{3}\left(2 \Delta_{A} \Delta_{A M}+2 \Delta_{C} \Delta_{S}+7 \Delta_{M} \Delta_{S O}+\Delta_{P S} \Delta_{V}\right)$,

$\beta_{\Delta_{S}}=-\epsilon_{d} \Delta_{S}+2 \Delta_{S}\left(\Delta_{A}-3 \Delta_{A M}+3 \Delta_{C}-3 \Delta_{M}+\Delta_{P S}-\Delta_{S}+3 \Delta_{S O}-\Delta_{V}\right)+4\left(2 \Delta_{A M} \Delta_{C}+\Delta_{M} \Delta_{P S}+\Delta_{S O} \Delta_{V}\right)$,

$\beta_{\Delta_{P S}}=-\epsilon_{d} \Delta_{P S}+2 \Delta_{P S}\left(\Delta_{A}+3 \Delta_{A M}+3 \Delta_{C}-3 \Delta_{M}-\Delta_{P S}+\Delta_{S}-3 \Delta_{S O}-\Delta_{V}\right)+4\left(\Delta_{A M} \Delta_{V}+2 \Delta_{C} \Delta_{S O}+\Delta_{M} \Delta_{S}\right)$.

The above set of coupled flow equations supports only a line of QCPs, given by Eq. (39), in the $\Delta_{V}-\Delta_{A}$ plane, shown in Fig. 10. Along the entire line of QPCs, the exponents are $\nu^{-1}=\epsilon_{d}$ and $z=1+\epsilon_{d} / 2$ (to the leading order in $\epsilon_{d}$ ). Therefore, in a three-dimensional WSM $\left(\epsilon_{d}=1\right)$ the semimetal-metal QPT driven by arbitrary disorder potential is always characterized by $\nu=1$ and $z=3 / 2$, thus strongly supporting the proposed emergent chiral superuniversality. Even though symmetry of a WSM is different from its two-dimensional counterpart graphene (for example, graphene does not allow the presence of timereversal symmetry-breaking magnetic or current disorder), at least the coupled RG flow equations for potential $\left(\Delta_{V}\right)$ and regular mass $\left(\Delta_{S}\right)$ disorder (present in both WSM and graphene) are in agreement with Ref. [84], if we set $\epsilon_{d}=0$ or equivalently $d=2$. 


\section{APPENDIX H: ALTERNATIVE DERIVATION OF CORRECTION TO OPTICAL CONDUCTIVITY}

Direct computation of the correction to the optical conductivity due to arbitrary disorder by using the Kubo formula has already been presented in Ref. [43]. Specifically, we compute the disorder-driven correction to the current-current correlation function (involving computation of two-loop diagrams), and then via analytic continuation we found the OC at frequency $\Omega$ in a weakly disordered WSM to be

$$
\sigma(\Omega)=\frac{N e_{0}^{2} \Omega}{12 h v}\left[1+\frac{\Delta_{V} \Lambda}{\pi^{2} v^{2}}\right] \equiv \sigma_{0}(\Omega)\left[1+\frac{\Delta_{V} \Lambda}{\pi^{2} v^{2}}\right],
$$

where $N$ is the number of Weyl nodes, and $e_{0}$ is the electron charge in vacuum [see Eq. (3) of Ref. [43] ]. For concreteness, we here restrict ourselves to potential disorder or random charge impurities $\left(\Delta_{V}\right)$, possessing Gaussian white noise distribution in three dimensions. In the absence of disorder $\left(\Delta_{V}=0\right)$, we recover the OC in a clean WSM, $\sigma_{0}(\Omega)[30,32,106]$. We now present an alternative derivation of the same expression.

The OC is given by

$$
\begin{aligned}
\sigma(\Omega) & =\lim _{\Omega \rightarrow 0} \frac{1}{\Omega} \int d^{D} x e^{i \Omega x_{0}}\left\langle j_{x}(x) j_{x}(0)\right\rangle_{R} \\
& =Z_{\Psi}^{2}\left[\lim _{\Omega \rightarrow 0} \frac{1}{\Omega} \int d^{D} x e^{i \Omega x_{0}}\left\langle j_{x}(x) j_{x}(0)\right\rangle_{0}\right] \\
& =Z_{\Psi}^{2}\left(\frac{N e_{0}^{2} \Omega}{12 h v}\right)
\end{aligned}
$$

where $Z_{\Psi}=\left[1+\Delta_{V} \Lambda /\left(2 \pi^{2} v^{2}\right)\right]$ is the field-renormalization factor, as presented in Sec. IV C, for $\epsilon_{d}=1$. The same expression for the field-renormalization factor can directly be obtained by integrating over the entire Weyl band with $0 \leq|\mathbf{k}| \leq \Lambda$, which is legitimate since we are interested in the OC of a weakly disordered WSM for which sharp quasiparticle excitations persist all the way down to zero energy or momentum. Upon substituting $Z_{\psi}$ in the above expression we immediately recover Eq. (H1).

\section{APPENDIX I: $\epsilon_{n}$ EXPANSION FOR WSM-METAL QPT}

We devote this appendix of the paper to address yet another controlled route to address the effects of disorder deep inside the WSM phase. Without any loss of generality we can express the Weyl Hamiltonian as

$$
H_{W}=v_{\perp} \sum_{j=1,2} i \gamma_{0} \gamma_{j} k_{j}+v_{3} i \gamma_{0} \gamma_{3} k_{3}
$$

and so far we have considered $v_{\perp}=v_{3}=v$. Following the spirit of the "band-flattening" method, demonstrated in Sec. III A, we deform the above Hamiltonian to

$$
H_{W} \rightarrow H_{W}^{n}=v_{\perp} \sum_{j=1,2} i \gamma_{0} \gamma_{j} k_{j}+C_{n} i \gamma_{0} \gamma_{3} k_{3}^{n},
$$

with the restriction that $n$ can now only take odd integer values, so that all symmetry properties of a WSM remain unaffected. The DOS of such a deformed system is $\varrho(E) \sim|E|^{1+1 / n}$. Notice in the limit $n \rightarrow \infty$ the DOS scales linearly with $E$, and disorder then become a marginal variable (outcome from a self-consistent Born calculation). Such a special limit represents a two-dimensional Weyl system (since quasiparticles do not possess any dispersion along $k_{z}$ ). Otherwise, following the same steps of coarse graining we find that the scaling dimension of disorder couplings after performing the disorder averaging using the replica formalism is $\left[\Delta_{j}\right]=-1 / n$. Therefore, we can perform a controlled RG calculation about the $n \rightarrow \infty$ limit, following the spirit of an $\epsilon_{n}$ expansion, with $\epsilon_{n}=1 / n$, since $\left[\Delta_{j}\right]=-\epsilon_{n}$. For the physically relevant case $\epsilon_{n}=1$. Otherwise, the steps are identical to the ones presented in Sec. III A and the relevant Feynman diagrams are already shown in Fig. 5. For the sake of simplicity, we here focus only on the potential disorder. A detailed RG analysis within the framework of the $\epsilon_{n}$ expansion in the presence of eight disorders is left for a future investigation. The leading-order RG calculation yields the following flow equations:

$$
\begin{aligned}
\beta_{X} & =-\Delta_{V} H_{0}(n) X=(1-z) X, \\
\beta_{\Delta_{V}} & =\Delta_{V}\left[-\epsilon_{n}+\Delta_{V} H_{0}(n)\right],
\end{aligned}
$$

where $X=v_{\perp}, C_{n}, \hat{\Delta}_{V}=2 \Delta_{V} \Lambda^{\epsilon_{n}} /\left[(2 \pi)^{2} C^{\epsilon_{n}} v_{\perp}^{2-\epsilon_{n}}\right]$ is the dimensionless disorder coupling, and for brevity we have dropped the hat notation in the last set of equations. The function $H_{0}(n)$ reads as

$$
H_{0}(n)=1+\frac{\pi^{2}}{24} \frac{1}{n^{2}}+\mathcal{O}\left(n^{-4}\right) .
$$

Therefore, $H_{0}(n)$ is a well-controlled function of $1 / n$. Keeping the leading-order term in $H_{0}(n)$, the RG equations become

$\beta_{X}=-\Delta_{V} X=(1-z) X, \quad \beta_{\Delta_{V}}=\Delta_{V}\left[-\epsilon_{n}+\Delta_{V}\right]$.

The DSE from the first equation reads as $z=1+\Delta_{V}$. The second equation supports only two fixed points: (i) the one at $\Delta_{V}=0$ represents the stable WSM phase, while (ii) the unstable fixed point at $\Delta_{V}=\epsilon_{n} / 2$ represents the WSMmetal QCP. The DSE and the CLE at this fixed point are, respectively,

$$
z=1+\frac{\epsilon_{n}}{2}, \quad \nu^{-1}=\epsilon_{n} .
$$

Therefore, for the physically relevant case of simple WSM $\left(\epsilon_{n}=1\right)$, we obtain $z=3 / 2$ and $\nu=1$, same as the ones obtained from $\epsilon_{m}$ and $\epsilon_{d}$ expansions, declared in Sec. V. 
Note that even if we chose to keep the entire function $H_{0}(n)$ in the RG flow equations, we obtain the same set of critical exponents.

\section{APPENDIX J: SELF-CONSISTENT BORN APPROXIMATION AT WSM-INSULATOR QCP}

In this appendix, we present the computation of the inverse scattering lifetime $\left(1 / \tau_{s}\right)$ within the framework of the self-consistent Born approximation, in the presence of disorder. In this formalism, $\tau_{s}$ is computed from the following self-consistent equation:

$$
\int_{0}^{E_{\Lambda}} d E \frac{\varrho(E)}{\left(\hbar / \tau_{s}\right)^{2}+E^{2}}=\frac{1}{W},
$$

where $E_{\Lambda}$ is the ultraviolet energy cutoff up to which critical excitations separating a WSM and an insulator possess anisotropic dispersion, captured by $H_{Q}(0)$ in Eq. (6). Since at the WSM-insulator QCP the average DOS scales as $\varrho(E) \sim|E|^{3 / 2}$, the right-hand side of the above equation displays ultraviolet divergence $\sim E_{\Lambda}^{1 / 2}$. Such divergence can be regulated by introducing a parameter

$$
\frac{1}{W_{c}}=\int_{0}^{E_{\Lambda}} d E \frac{\varrho(E)}{E^{2}},
$$

where $W_{c}$ corresponds to the critical strength of disorder for the instability of ballistic critical fermions. The above gap equation can then be cast as

$$
\delta=\int_{0}^{E_{\Lambda}} d E \varrho(E)\left(\frac{1}{E^{2}}-\frac{1}{\left(\hbar / \tau_{s}\right)^{2}+E^{2}}\right),
$$

where $\delta=W-W_{c} /\left(W W_{c}\right)$ measures the reduced disorder strength from the critical one $\left(W=W_{c}\right)$. After regularizing the ultraviolet divergence we can take the limit $E_{\Lambda} \rightarrow \infty$ without encountering any divergence. The self-consistent solution of the scattering lifetime is then obtained from the following universal scaling form,

$$
\sqrt{\frac{\hbar}{\tau_{s}}}=\frac{\sqrt{2}}{\pi} \delta,
$$

which immediately implies that $\tau_{s}^{-1}$ is finite only when $\delta>0$ or $W>W_{c}$, and for $W<W_{c}$ we get $\tau_{s}^{-1}=0$. Therefore, critical fermions separating a WSM and an insulator retain its ballistic nature up to a critical strength of disorder $W_{c} \sim E_{\Lambda}^{1 / 2}$. Only for strong disorder $W>W_{c}$ a metallic phase emerges where $\tau_{s}^{-1}$ is finite. Therefore, the conclusion from the self-consistent Born approximation is in qualitative agreement with our results found by fieldtheoretic RG analysis and numerical calculation, presented in Sec. III.
[1] C. Herring, Accidental Degeneracy in the Energy Bands of Crystals, Phys. Rev. 52, 365 (1937).

[2] R. Dornhaus, G. Nimtz, and B. Schlicht, Narrow-Gap Semicounductors (Springer-Verlag, Berlin, 1983).

[3] G. E. Volovik, The Universe in a Helium Droplet (Oxford University Press, New York, 2003).

[4] T. O. Wehling, A. M. Black-Schaffer, and A. V. Balatsky, Dirac Materials, Adv. Phys. 63, 1 (2014).

[5] C.-K. Chiu, J. C. Y. Teo, A.P. Schnyder, and S. Ryu, Classification of Topological Quantum Matter with Symmetries, Rev. Mod. Phys. 88, 035005 (2016).

[6] A. Bansil, Hsin Lin, and Tanmoy Das, Colloquium: Topological Band Theory, Rev. Mod. Phys. 88, 021004 (2016).

[7] B. Bradlyn, J. Cano, Z. Wang, M. G. Vergniory, C. Felser, R. J. Cava, and B. A. Bernevig, Beyond Dirac and Weyl Fermions: Unconventional Quasiparticles in Conventional Crystals, Science 353, aaf5037 (2016).

[8] B. J. Wieder and C. L. Kane, Spin-Orbit Semimetals in the Layer Groups, Phys. Rev. B 94, 155108 (2016).

[9] R.-J. Slager, V. Juricic, V. Lahtinen, and J. Zaanen, SelfOrganized Pseudo-Graphene on Grain Boundaries in Topological Band Insulators, Phys. Rev. B 93, 245406 (2016).

[10] P. A. M. Dirac, The Quantum Theory of the Electron, Proc. R. Soc. A 117, 610 (1928).

[11] P. A. M. Dirac, A Theory of Electrons and Protons, Proc. R. Soc. A 126, 360 (1930).

[12] H. Weyl, Elektron und Gravitation. I, Z. Phys. 56, 330 (1929).

[13] A. A. Burkov, Chiral Anomaly and Transport in Weyl Metals, J. Phys. Condens. Matter 27, 113201 (2015).

[14] S. Rao, Weyl Semi-Metals: A Short Review, arXiv:1603 .02821 .

[15] N. P. Armitage, E. J. Mele, and A. Vishwanath, Weyl and Dirac Semimetals in Three Dimensional Solids, Rev. Mod. Phys. 90, 015001 (2018).

[16] C. Zhang, Z. Yuan, S. Xu, Z. Lin, B. Tong, M. Z. Hasan, J. Wang, C. Zhang, and S. Jia, Electron Scattering in Tantalum Monoarsenide, Phys. Rev. B 95, 085202 (2017).

[17] S.-Y. Xu, I. Belopolski, N. Alidoust, M. Neupane, C. Zhang, R. Sankar, S.-M. Huang, C.-C. Lee, G. Chang, B. Wang, G. Bian, H. Zheng, D. S. Sanchez, F. Chou, H. Lin, S. Jia, and M.Z. Hasan, Discovery of a Weyl Fermion Semimetal and Topological Fermi Arcs, Science 349, 613 (2015).

[18] B. Q. Lv, H. M. Weng, B. B. Fu, X. P. Wang, H. Miao, J. Ma, P. Richard, X. C. Huang, L. X. Zhao, G. F. Chen, Z. Fang, X. Dai, T. Qian, and H. Ding, Experimental Discovery of Weyl Semimetal TaAs, Phys. Rev. X 5, 031013 (2015).

[19] S.-Y. Xu et al., Discovery of a Weyl Fermion State with Fermi Arcs in Niobium Arsenide, Nat. Phys. 11, 748 (2015).

[20] N. Xu et al., Observation of Weyl Nodes and Fermi Arcs in Tantalum Phosphide, Nat. Commun. 7, 11006 (2016).

[21] C. Shekhar, A. K. Nayak, Y. Sun, M. Schmidt, M. Nicklas, I. Leermakers, U. Zeitler, Z. Liu, Y. Chen, W. Schnelle, J. Grin, C. Felser, and B. Yan, Extremely Large 
Magnetoresistance and Ultrahigh Mobility in the Topological Weyl Semimetal Candidate NbP, Nat. Phys. 11, 645 (2015).

[22] Z. Wang, Y. Zheng, Z. Shen, Y. Zhou, X. Yang, Y. Li, C. Feng, and Z.-A. Xu, Helicity-Protected Ultrahigh Mobility Weyl Fermions in NbP, Phys. Rev. B 93, 121112 (2016).

[23] G. Chang, S.-Y. Xu, D. S. Sanchez, S.-M. Huang, C.-C. Lee, T.-R. Chang, H. Zheng, G. Bian, I. Belopolski, N. Alidoust, H.-T. Jeng, A. Bansil, H. Lin, and M. Z. Hasan, A Strongly Robust Type II Weyl Fermion Semimetal State in $\mathrm{Ta}_{2} \mathrm{~S}_{3}$, Sci. Adv. 2, e1600295 (2016).

[24] S. Borisenko, D. Evtushinsky, Q. Gibson, A. Yaresko, T. Kim, M. N. Ali, B. Buechner, M. Hoesch, and R. J. Cava, Time-Reversal Symmetry Breaking Type-II Weyl State in $\mathrm{YbMnBi}_{2}$, arXiv:1507.04847.

[25] J. Y. Liu, J. Hu, Q. Zhang, D. Graf, H. B. Cao, S. M. A. Radmanesh, D. J. Adams, Y. L. Zhu, G. F. Cheng, X. Liu, W. A. Phelan, J. Wei, D. A. Tennant, J. F. DiTusa, I. Chiorescu, L. Spinu, and Z. Q. Mao, Discovery of a Topological Semimetal Phase Coexisting with Ferromagnetic Behavior in $\mathrm{Sr}_{1-y} \mathrm{MnSb}_{2}(y \sim 0.08)$, Nat. Mater. 16, 905 (2017).

[26] H. B. Nielsen and M. Ninomiya, Absence of Neutrinos on a Lattice: (I). Proof by Homotopy Theory, Nucl. Phys. B185, 20 (1981); A No-Go Theorem for Regularizing Chiral Fermions, Phys. Lett. B 105, 219 (1981).

[27] A. B. Harris, Effect of Random Defects on the Critical Behaviour of Ising Models, J. Phys. C 7, 1671 (1974).

[28] E. Fradkin, Critical Behavior of Disordered Degenerate Semiconductors. II. Spectrum and Transport Properties in Mean-Field Theory, Phys. Rev. B 33, 3263 (1986).

[29] R. Shindou and S. Murakami, Effects of Disorder in ThreeDimensional $\mathrm{Z}_{2}$ Quantum Spin Hall Systems, Phys. Rev. B 79, 045321 (2009).

[30] P. Goswami and S. Chakravarty, Quantum Criticality between Topological and Band Insulators in $3+1$ Dimensions, Phys. Rev. Lett. 107, 196803 (2011).

[31] S. Ryu and K. Nomura, Disorder-Induced Quantum Phase Transitions in Three-Dimensional Topological Insulators and Superconductors, Phys. Rev. B 85, 155138 (2012).

[32] P. Hosur, S. A. Parameswaran, and A. Vishwanath, Charge Transport in Weyl Semimetals, Phys. Rev. Lett. 108, 046602 (2012).

[33] Z. Huang, T. Das, A. V. Balatsky, and D. P. Arovas, Stability of Weyl Metals under Impurity Scattering, Phys. Rev. B 87, 155123 (2013).

[34] R. Nandkishore, D. A. Huse, and S. L. Sondhi, Rare Region Effects Dominate Weakly Disordered ThreeDimensional Dirac Points, Phys. Rev. B 89, 245110 (2014).

[35] Y. Ominato and M. Koshino, Quantum Transport in a Three-Dimensional Weyl Electron System, Phys. Rev. B 89, 054202 (2014); Quantum Transport in ThreeDimensional Weyl Electron System in the Presence of Charged Impurity Scattering, Phys. Rev. B 91, 035202 (2015).

[36] B. Roy and S. Das Sarma, Diffusive Quantum Criticality in Three-Dimensional Disordered Dirac Semimetals, Phys. Rev. B 90, 241112(R) (2014).
[37] S. V. Syzranov, L. Radzihovsky, and V. Gurarie, Critical Transport in Weakly Disordered Semiconductors and Semimetals, Phys. Rev. Lett. 114, 166601 (2015); S. V. Syzranov, V. Gurarie, and L. Radzihovsky, Unconventional Localization Transition in High Dimensions, Phys. Rev. B 91, 035133 (2015).

[38] E.-G. Moon and Y.-B. Kim, Non-Fermi Liquid in Dirac Semi-Metals, arXiv:1409.0573.

[39] A. Altland and D. Bagrets, Effective Field Theory of the Disordered Weyl Semimetal, Phys. Rev. Lett. 114, 257201 (2015).

[40] B. Roy and S. Das Sarma, Erratum: Diffusive Quantum Criticality in Three-Dimensional Disordered Dirac Semimetals, Phys. Rev. B 90, 241112(R) (2014); 93, 119911(E) (2016).

[41] S. V. Syzranov, P. M. Ostrovsky, V. Gurarie, and L. Radzihovsky, Critical Exponents at the Unconventional Disorder-Driven Transition in a Weyl Semimetal, Phys. Rev. B 93, 155113 (2016).

[42] B. Roy and S. Das Sarma, Quantum Phases of Interacting Electrons in Three-Dimensional Dirty Dirac Semimetals, Phys. Rev. B 94, 115137 (2016).

[43] B. Roy, V. Juričić, and S. Das Sarma, Universal Optical Conductivity of a Disordered Weyl Semimetal, Sci. Rep. 6, 32446 (2016).

[44] P. Goswami and S. Chakravarty, Superuniversality of Topological Quantum Phase Transition and Global Phase Diagram of Dirty Topological Systems in Three Dimensions, Phys. Rev. B 95, 075131 (2017).

[45] T. Louvet, D. Carpentier, and A. A. Fedorenko, On the Disorder-Driven Quantum Transition in ThreeDimensional Relativistic Metals, Phys. Rev. B 94, 220201(R) (2016).

[46] S. V. Syzranov, V. Gurarie, and L. Radzihovsky, Multifractality at Non-Anderson Disorder-Driven Transitions in Weyl Semimetals and Other Systems, Ann. Phys. (Amsterdam) 373, 694 (2016).

[47] A. K. Mitchell and L. Fritz, Signatures of Weyl Semimetals in Quasiparticle Interference, Phys. Rev. B 93, 035137 (2016).

[48] E. V. Gorbar, V. A. Miransky, I. A. Shovkovy, and P. O. Sukhachov, Origin of Dissipative Fermi Arc Transport in Weyl Semimetals, Phys. Rev. B 93, 235127 (2016).

[49] M. J. Park, B. Basa, and M. J. Gilbert, Disorder-Induced Phase Transitions of Type-II Weyl Semimetals, Phys. Rev. B 95, 094201 (2017).

[50] T. Louvet, D. Carpentier, and A. A. Fedorenko, New Quantum Transition in Weyl Semimetals with Correlated Disorder, Phys. Rev. B 95, 014204 (2017).

[51] K. Kobayashi, T. Ohtsuki, and K.-I. Imura, Disordered Weak and Strong Topological Insulators, Phys. Rev. Lett. 110, 236803 (2013).

[52] K. Kobayashi, T. Ohtsuki, K.-I. Imura, and I. F. Herbut, Density of States Scaling at the Semimetal to Metal Transition in Three Dimensional Topological Insulators, Phys. Rev. Lett. 112, 016402 (2014).

[53] B. Sbierski, G. Pohl, E. J. Bergholtz, and P. W. Brouwer, Quantum Transport of Disordered Weyl Semimetals at the Nodal Point, Phys. Rev. Lett. 113, 026602 (2014). 
[54] J. H. Pixley, P. Goswami, and S. Das Sarma, Anderson Localization and the Quantum Phase Diagram of Three Dimensional Disordered Dirac Semimetals, Phys. Rev. Lett. 115, 076601 (2015).

[55] B. Sbierski, E. J. Bergholtz, and P. W. Brouwer, Quantum Critical Exponents for a Disordered Three-Dimensional Weyl Node, Phys. Rev. B 92, 115145 (2015).

[56] J. H. Pixley, P. Goswami, and S. Das Sarma, Disorder-Driven Itinerant Quantum Criticality of Three-Dimensional Massless Dirac Fermions, Phys. Rev. B 93, 085103 (2016).

[57] S. Liu, T. Ohtsuki, and R. Shindou, Effect of Disorder in a Three-Dimensional Layered Chern Insulator, Phys. Rev. Lett. 116, 066401 (2016).

[58] C.-Z. Chen, J. Song, H. Jiang, Q.-F. Sun, Z. Wang, and X. C. Xie, Disorder and Metal-Insulator Transitions in Weyl Semimetals, Phys. Rev. Lett. 115, 246603 (2015).

[59] S. Bera, J. D. Sau, and B. Roy, Dirty Weyl Semimetals: Stability, Phase Transition, and Quantum Criticality, Phys. Rev. B 93, 201302 (2016).

[60] H. Shapourian and T.L. Hughes, Phase Diagrams of Disordered Weyl Semimetals, Phys. Rev. B 93, 075108 (2016).

[61] J. H. Pixley, D. A. Huse, and S. Das Sarma, Rare-RegionInduced Avoided Quantum Criticality in Disordered Three-Dimensional Dirac and Weyl Semimetals, Phys. Rev. X 6, 021042 (2016).

[62] B. Roy, Y. Alavirad, and J. D. Sau, Global Phase Diagram of a Three-Dimensional Dirty Topological Superconductor, Phys. Rev. Lett. 118, 227002 (2017).

[63] J. H. Pixley, D. A. Huse, and S. Das Sarma, Uncovering the Hidden Quantum Critical Point in Disordered Massless Dirac and Weyl Semimetals, Phys. Rev. B 94, 121107(R) (2016).

[64] Y. Takane, Disorder Effect on Chiral Edge Modes and Anomalous Hall Conductance in Weyl Semimetals, J. Phys. Soc. Jpn. 85, 124711 (2016).

[65] R.-J. Slager, V. Juričić, and B. Roy, Dissolution of Topological Fermi Arcs in a Dirty Weyl Semimetal, Phys. Rev. B 96, 201401 (2017).

[66] B. Roy and J. D. Sau, Magnetic Catalysis and Axionic Charge Density Wave in Weyl Semimetals, Phys. Rev. B 92, 125141 (2015).

[67] Throughout this paper, we use chiral-symmetric and intranode disorder synonymously. We also use chiral symmetry breaking and internode disorder synonymously. However, such classification is only germane for infinitesimal strength of randomness. At strong disorder all possible types of randomness are generated, leading to the notion of emergent superuniversality across the disorderdriven WSM-metal QPT.

[68] C.-X. Liu, X.-L. Qi, H.-J. Zhang, X. Dai, Z. Fang, and S.-C. Zhang, Model Hamiltonian for Topological Insulators, Phys. Rev. B 82, 045122 (2010).

[69] C.-X. Liu, P. Ye, and X.-L. Qi, Chiral Gauge Field and Axial Anomaly in a Weyl Semimetal, Phys. Rev. B 87, 235306 (2013).

[70] P. Goswami and B. Roy, Effective Field Theory, Chiral Anomaly and Vortex Zero Modes for Odd Parity Topological Superconducting State of Three Dimensional Dirac Materials, arXiv:1211.4023, and references therein.
[71] X. Li, B. Roy, and S. Das Sarma, Weyl Fermions with Arbitrary Monopoles in Magnetic Fields: Landau Levels, Longitudinal Magnetotransport, and Density-Wave Ordering, Phys. Rev. B 94, 195144 (2016).

[72] A. Weiße, G. Wellein, A. Alverman, and H. Feshke, The Kernel Polynomial Method, Rev. Mod. Phys. 78, 275 (2006).

[73] S. Kivelson, D.-H. Lee, and S.-C. Zhang, Global Phase Diagram in the Quantum Hall Effect, Phys. Rev. B 46, 2223 (1992).

[74] C. A. Lütken and G. G. Ross, Delocalization, Duality, and Scaling in the Quantum Hall System, Phys. Rev. B 48, 2500 (1993).

[75] E. Fradkin and S. Kivelson, Modular Invariance, SelfDuality and the Phase Transition between Quantum Hall Plateaus, Nucl. Phys. B474, 543 (1996).

[76] I. A. Gruzberg, N. Read, and S. Vishveshwara, Localization in Disordered Superconducting Wires with Broken Spin-Rotation Symmetry, Phys. Rev. B 71, 245124 (2005).

[77] For a comprehensive discussion on Anderson transition and See 50 Years of Anderson Localization, 1st ed., edited by E. Abrahams (World Scientific Publishing Company, Singapore, 2010).

[78] R.-J. Slager, A. Mesaros, V. Juricic, and J. Zaanen, The Space Group Classification of Topological BandInsulators, Nat. Phys. 9, 98 (2013).

[79] J. Zinn-Justin, Quantum Field Theory and Critical Phenomena (Oxford University Press, Oxford, 2002).

[80] B. Roy, P. Goswami, and V. Juričić, Interacting Weyl Fermions: Phases, Phase Transitions, and Global Phase Diagram, Phys. Rev. B 95, 201102(R) (2017).

[81] B. Roy and M. S. Foster, Quantum Multicriticality near the Dirac-Semimetal to Band-Insulator Critical Point in Two Dimensions: A Controlled Ascent from One Dimension, Phys. Rev. X 8, 011049 (2018).

[82] S. Sachdev, Quantum Phase Transitions, 2nd ed. (Cambridge University Press, Cambridge, England, 2007).

[83] See A. Weinrib and B. I. Halperin, Critical Phenomena in Systems with Long-Range-Correlated Quenched Disorder, Phys. Rev. B 27, 413 (1983) for general discussion on correlated disorder.

[84] P. M. Ostrovsky, I. V. Gornyi, and A. D. Mirlin, Electron Transport in Disordered Graphene, Phys. Rev. B 74, 235443 (2006).

[85] We note that the quality of the data collapses for CSB disorders, shown in Fig. 14, is slightly less pronounced than those for CSP disorder, displayed in Fig. 13, which can qualitatively be understood in the following way. In the presence of only internode scatterers, the system first tends to flow toward the line of QCPs set by purely CSB disorder, discussed early in this section. Only when disorder gets sufficiently strong the intranode disorder becomes relevant and the system starts flowing toward the line of QCPs discussed in Sec. V. The system then gets stuck in the crossover regime dominated by CSB disorder, and consequently the data collapse (involving finite energy states) becomes slightly less prominent. To achieve equally good quality data collapse even in the presence of CSB disorder, we therefore need to subscribe to larger systems, which can be numerically challenging. 
[86] In spite of the emergent superuniversality, the putative line of QCPs driven by CSB disorders with continuously varying DSE $z>d$ may leave its imprint on the physical observables in the crossover regime before the CSP disorders take over and ultimately the system flows toward the chiral symmetric quantum-critical line with $z=3 / 2$ and $\nu=1$. In that sense the physical observables we discuss in this section can also distinguish between different types of disorder (internode versus intranode).

[87] I. F. Herbut, V. Juričić, and B. Roy, Theory of Interacting Electrons on the Honeycomb Lattice, Phys. Rev. B 79, 085116 (2009).

[88] Y. Otsuka, S. Yunoki, and S. Sorella, Universal Quantum Criticality in the Metal-Insulator Transition of TwoDimensional Interacting Dirac Electrons, Phys. Rev. X 6, 011029 (2016).

[89] J. H. Pixley, Y.-Z. Chou, P. Goswami, D. A. Huse, R. Nandkishore, L. Radzihovsky, and S. Das Sarma, SingleParticle Excitations in Disordered Weyl Fluids, Phys. Rev. B 95, 235101 (2017).

[90] F. Wegner, Electrons in Disordered Systems. Scaling near the Mobility Edge, Z. Phys. B 25, 327 (1976).

[91] D. Belitz and T.R. Kirkpatrick, The Anderson-Mott transition, Rev. Mod. Phys. 66, 261 (1994).

[92] F. Evers and A. D. Mirlin, Anderson Transitions, Rev. Mod. Phys. 80, 1355 (2008).

[93] M. Janssen, Statistics and Scaling in Disordered Mesoscopic Electron Systems, Phys. Rep. 295, 1 (1998).

[94] M.S. Foster, Multifractal Nature of the Surface Local Density of States in Three-Dimensional Topological Insulators with Magnetic and Nonmagnetic Disorder, Phys. Rev. B 85, 085122 (2012).

[95] J. Brndiar and P. Markoš, Universality of the Metal-Insulator Transition in Three-Dimensional Disordered Systems, Phys. Rev. B 74, 153103 (2006).

[96] S. Borisenko, Q. Gibson, D. Evtushinsky, V. Zabolotnyy, B. Buechner, and R. J. Cava, Experimental Realization of a Three-Dimensional Dirac Semimetal, Phys. Rev. Lett. 113, 027603 (2014).
[97] Z. K. Liu, B. Zhou, Y. Zhang, Z. J. Wang, H. M. Weng, D. Prabhakaran, S.-K. Mo, Z. X. Shen, Z. Fang, X. Dai, Z. Hussain, and Y.L. Chen, Discovery of a ThreeDimensional Topological Dirac Semimetal, $\mathrm{Na}_{3} \mathrm{Bi}$, Science 343, 864 (2014).

[98] I. Balog, D. Carpentier, and A. A. Fedorenko, DisorderDriven Quantum Transition in Relativistic Semimetals: Functional Renormalization via the Porous Medium Equation, arXiv:1710.07932.

[99] S. Das Sarma, S. Adam, E. H. Hwang, and E. Rossi, Electronic Transport in Two-Dimensional Graphene, Rev. Mod. Phys. 83, 407 (2011).

[100] B. I. Halperin and M. Lax, Impurity-Band Tails in the High-Density Limit. I. Minimum Counting Methods, Phys. Rev. 148, 722 (1966).

[101] G. Xu, H. Weng, Z. Wang, X. Dai, and Z. Fang, Chern Semimetal and the Quantized Anomalous Hall Effect in $\mathrm{HgCr}_{2} \mathrm{Se}_{4}$, Phys. Rev. Lett. 107, 186806 (2011).

[102] C. Fang, M. J. Gilbert, X. Dai, and B. A. Bernevig, Multi-Weyl Topological Semimetals Stabilized by Point Group Symmetry, Phys. Rev. Lett. 108, 266802 (2012).

[103] B.-J. Yang and N. Nagaosa, Classification of Stable Three-Dimensional Dirac Semimetals with Nontrivial Topology, Nat. Commun. 5, 4898 (2014).

[104] D. Carpentier, A. A. Fedorenko, and E. Orignac, Effect of Disorder on 2D Topological Merging Transition from a Dirac Semi-Metal to a Normal Insulator, Eur. Phys. Lett. 102, 67010 (2013).

[105] B. Sbierski, M. Trescher, E. J. Bergholtz, and P. W. Brouwer, Disordered Double Weyl Node: Comparison of Transport and Density of States Calculations, Phys. Rev. B 95, 115104 (2017).

[106] B. Roy and V. Juričić, Optical Conductivity of an Interacting Weyl Liquid in the Collisionless Regime, Phys. Rev. B 96, 155117 (2017); Collisionless Transport Close to a Fermionic Quantum Critical Point in Dirac Materials, arXiv:1801.03495 [Phys. Rev. Lett. (to be published)]. 